\title{
LARGE SOLUTIONS AND GRADIENT BOUNDS FOR QUASILINEAR ELLIPTIC EQUATIONS
}

\author{
TOMMASO LEONORI AND ALESSIO PORRETTA
}

Abstract. We consider the quasilinear degenerate elliptic equation

$$
\lambda u-\Delta_{p} u+H(x, D u)=0 \quad \text { in } \Omega
$$

where $\Delta_{p}$ is the $p$-Laplace operator, $p>2, \lambda \geq 0$ and $\Omega$ is a smooth open bounded subset of $\mathbb{R}^{N}(N \geq 2)$. Under suitable structure conditions on the function $H$, we prove local and global gradient bounds for the solutions. We apply these estimates to study the solvability of the Dirichlet problem, and the existence, uniqueness and asymptotic behavior of maximal solutions blowing up at the boundary. The ergodic limit for those maximal solutions is also studied and the existence and uniqueness of a so-called additive eigenvalue is proved in this context.

\section{Contents}

1. Introduction

2. Main results of the paper 5

2.1. Global gradient bounds 5

$\begin{array}{llr}2.2 . & \text { Interior gradient bounds } & 8\end{array}$

\begin{tabular}{llr}
\hline 2.3. & The Dirichlet problem & 9
\end{tabular}

\begin{tabular}{lr}
2.4. & Large solutions \\
\hline
\end{tabular}

\begin{tabular}{llr}
\hline 2.5. & The ergodic problem & 12 \\
\hline
\end{tabular}

3. Proof of the results on the Dirichlet problem 14

\begin{tabular}{|lr}
\hline 4. & Proof of the results on large solutions \\
\hline
\end{tabular}

\begin{tabular}{ll}
\hline 4.1. & Existence \\
\hline
\end{tabular}

\begin{tabular}{ll}
4.2. & Uniqueness \\
\hline
\end{tabular}

5. Proof of the results on the ergodic problem 24

$\begin{array}{ll}\text { 6. Proof of the gradient bounds } & 28\end{array}$

6.1. Global gradient estimates 29

6.2. Local gradient estimates 33

\begin{tabular}{llr}
\hline 7. Neumann boundary conditions & 38 \\
\hline
\end{tabular}

$\begin{array}{lr}\text { References } & 40\end{array}$

\section{INTRODUCTION}

In this paper we deal with several questions concerning the existence of solutions to quasilinear degenerate elliptic equations of the type
$\left(E_{\lambda}\right)$
$\lambda u-\Delta_{p} u+H(x, D u)=0 \quad$ in $\Omega$,

Date: October 9, 2015. 
where $\Omega$ is an open bounded subset of the euclidean space $\mathbb{R}^{N}(N \geq 1)$, which is assumed to have smooth boundary (say, of class $C^{2}$ ). In $\left.\sqrt{E_{\lambda}}\right) u: \Omega \mapsto \mathbb{R}$ is a scalar function ( $D u$ denotes its gradient vector), $\lambda \in \mathbb{R}, \lambda \geq 0, H: \Omega \times \mathbb{R}^{N} \rightarrow \mathbb{R}$ is a function satisfying suitable regularity and growth conditions to be given below, and $\Delta_{p} u$ denotes the classical $p$-Laplace operator, i.e.

$$
\Delta_{p} u=\operatorname{div}\left(|D u|^{p-2} D u\right) .
$$

We assume that $p>2$, so that the ellipticity of the operator may degenerate. One of the main points in our study is concerned with the Dirichlet problem associated with $\left(E_{\lambda}\right)$ :

$$
\begin{cases}\lambda u-\Delta_{p} u+H(x, D u)=0 & \text { in } \Omega \\ u=0 & \text { on } \partial \Omega .\end{cases}
$$

It is well-known that the solvability of 1.1 depends, in primis, on the growth of the function $H(x, \xi)$ with respect to $\xi$ and, secondly, on the case whether $\lambda>0$ or $\lambda=0$.

When $p=2$, the question of the existence of (classical) solutions to 1.1 has largely been studied and the crucial role of some ingredients has emerged so far: the existence of (local, global or universal) a priori bounds for the gradient of solutions, the behavior of the maximal solutions to $\left(E_{\lambda}\right)$, the ergodic constant associated to those maximal solutions (and the state constraint problem). Among the several contributions to this issue, which would be impossible here to mention all, let us refer the reader to [34, 29], 30, 18] for the development of gradient estimates applied to the solution of boundary value problems, to 20 for a study of maximal solutions and the associated ergodic state constraint problem for the Brownian motion, and to [10, 32, 37. for the characterization, in terms of the ergodic constant, of the solvability of the Dirichlet problem when $\lambda=0$ and the long time behavior of the evolution problem.

The goal of this paper is to extend many of the above mentioned results to the case of the $p$-Laplace operator with $p>2$. To this purpose, we investigate several topics which are mutually correlated: the local and global Lipschitz estimates for $\left(E_{\lambda}\right)$, the maximal solutions to $E_{\lambda}$ blowing up at the boundary, the corresponding ergodic problem or, otherwise said, the existence and uniqueness of a nonlinear additive eigenvalue for the case $\lambda=0$. The correlation among those three topics is concerned with the solvability of the Dirichlet problem (1.1).

The first topic we discuss is the existence of gradient bounds for solutions to $E_{\lambda}$. This is a classical question in the context of quasilinear equations with first order terms, as explained e.g. in [18, Chapter 15], and it has largely been studied in the case $p=2$ since the pioneering paper by S.Bernstein (see [12]) and the nowadays classical results by J.Serrin, P-L. Lions and G.Barles ([34, 6], 28, 29], 30], and references cited therein). Along the lines of those results, we extend here those estimates to $p$-Laplace type equations. Despite some results were obtained in this direction (see e.g. 3], 4], 11, and references cited therein) it seems, at least to our knowledge, that general criteria for the obtention of gradient bounds had not been developed so far for the case $p>2$. By adapting the techniques of [29, 30, we prove two type of results under general structure conditions.

The first one is a global estimate that bounds the gradient of the solution with the value of the gradient at $\partial \Omega$ and with the $L^{\infty}$ bound of the solution. Namely, an estimate of the type

$$
\sup _{\bar{\Omega}}|D u|^{2} \leq c+\sup _{\partial \Omega}|D u|^{2},
$$

where $c=c\left(\|u\|_{L^{\infty}(\Omega)}\right)$. This estimate is proved assuming $H(x, \xi) \in C^{1}\left(\Omega \times \mathbb{R}^{N}\right)$ and one between two possible structure conditions, 2.3$)-(2.4)$ or $(2.6)$, which are discussed in detail in the next section. Let us stress that in this result the Hamiltonian is not a priori restricted to 
have natural growth, this is where the gradient bounds obtained are different from the classical $C^{1, \alpha}$ estimates for the $p$-Laplacian, see e.g. [16] and [26].

The second type of result that we prove is an interior gradient bound, namely that for any $\omega \subset \subset \Omega$

$$
\sup _{x \in \omega}|D u| \leq c_{\omega}
$$

for some constant $c_{\omega}$ which only depends on $\lambda\left\|u^{-}\right\|_{L^{\infty}\left(\omega^{\prime}\right)}$, for any $\omega \subset \subset \omega^{\prime} \subseteq \Omega$. This estimate, which is completely independent of the boundary behavior of the solutions, is proved assuming that the first order term is coercive, meaning that

$$
H(x, \xi) \geq h\left(|\xi|^{p-1}\right)-C_{\omega}, \quad x \in \omega
$$

for some increasing convex function $h(s)$ such that $\int^{\infty} \frac{d \tau}{h(\tau)}<\infty$. A few more technical assumptions are also needed, which are detailed in the next section.

Actually, the above global or local estimates are proved to hold for the solutions $u_{\varepsilon}$ of the uniformly elliptic problem

$$
\lambda u_{\varepsilon}-\operatorname{div}\left(\left(\varepsilon+\left|D u_{\varepsilon}\right|^{2}\right)^{\frac{p-2}{2}} D u_{\varepsilon}\right)+H\left(x, D u_{\varepsilon}\right)=0 \quad \text { in } \Omega,
$$

with a bound independent of $\varepsilon$. Those uniform estimates obtained for 1.4 imply the desired global or local Lipschitz regularity for the solutions to $E_{\lambda}$ obtained in the limit. Standard uniqueness results may be used to deduce that the estimates hold for weak solutions of boundary value problems.

The main application that we give of the global estimate $[1.2$ is concerned with the solvability of the Dirichlet problem (1.1). First of all, we extend the existence criteria given by P.-L. Lions (see [29) in the case $p=2$ and $H(x, \cdot)$ convex, that a solution of (1.1) exists provided one finds a Lipschitz (strong) subsolution to the same problem. To be more precise, we first prove that, under the structure conditions mentioned above, if $H$ is bounded below the existence of a $W_{0}^{1, \infty}(\Omega)$ sub solution implies the existence of a $W_{0}^{1, \infty}(\Omega)$ solution to 1.1 .

We are led so far to the question whether $W_{0}^{1, \infty}(\Omega)$ sub solutions can be found or not. Here, two kind of regimes are observed depending on the behavior of the function $H(x, \cdot)$ at infinity. Roughly speaking, if

$$
H(x, \xi) \simeq h\left(|\xi|^{p-1}\right) \quad \text { as }|\xi| \rightarrow \infty,
$$

then two possible ranges can occur:

(i) if $\int^{\infty} \frac{\tau^{\frac{1}{p-1}}}{h(\tau)} d \tau=\infty$, there always exist local Lipschitz barriers at the boundary; as a consequence, for the existence of a solution to (1.1) it is enough to find a merely bounded sub solution. In particular, when $\lambda>0$ then (1.1) always admits a (globally Lipschitz) solution, and when $\lambda=0$ solutions can be lost only because of $L^{\infty}$-blow up.

(ii) if $\int^{\infty} \frac{\tau^{\frac{1}{p-1}}}{h(\tau)} d \tau<\infty$, local Lipschitz barriers at the boundary cannot be constructed with arbitrary large sup-norm. In this case, solutions to 1.1 can be lost even if $\lambda>0$ because a gradient blow-up may happen without blow-up of the $L^{\infty}$ norm.

Let us stress that the two alternative behaviors (i) and (ii) described above were previously found in [2] for the case $p=2$ in the study of the model problem in which $H(x, \xi)=h(|\xi|)-f(x)$. Notice that, if $p=2$, the above integral threshold distinguishes -at least in the power scalebetween the so-called natural growth and a "super quadratic" growth of the Hamiltonian. In this latter case, phenomena of gradient blow-up, loss of boundary conditions, and universal upper 
bounds were extensively described, see e.g. [7, [14, 15, [37 for stationary equations. For $p$ Laplace type equations, the range corresponding to the case (ii) above was recently studied in [5] and we refer to this paper for further references.

The main application that we give of the interior gradient bound $\sqrt{1.3}$ is the study of the maximal solutions to equation $\left(E_{\lambda}\right)$ in $\Omega$. The behavior of those maximal solutions also changes according to the ranges (i) and (ii) mentioned above. For the sake of brevity, we focus our attention to the simplest case in which $H(x, \xi)$ has a power-type growth with respect to $|\xi|$, at least near the boundary. Roughly speaking, we will assume that near the boundary $H(x, \xi)=$ $O\left(|\xi|^{q}\right)$ with $p-1<q \leq p$. In this case the maximal solutions blow-up at the boundary and equation $E_{\lambda}$ is complemented with the boundary blow-up condition

$$
\lim _{x \rightarrow \partial \Omega} u(x)=+\infty .
$$

In literature, solutions to $E_{\lambda}-1.5$ are often called large solutions. Observe that the limitation $q>p-1$ is needed in order to have the barrier effects and the interior estimates, while the limitation $q \leq p$ is required to have that maximal solutions actually blow-up at the boundary. If $q>p$, maximal solutions would remain bounded in view of global Hölder bounds (see e.g. 14), for the study of maximal solutions in this case, and in the more general range (ii) mentioned above, we refer again to $[5$.

Concerning solutions to $E_{\lambda}$ and 1.5 , we first provide a result of existence and uniqueness when $\lambda>0$ (see Theorem 2.11), then we study the limit of solutions $u_{\lambda}$ as $\lambda$ tends to zero (so-called ergodic limit in the case $p=2$ ). Here we fully exploit the relevance of estimate 1.3 ) which remains uniform as $\lambda \rightarrow 0$, despite the fact that the global $L^{\infty}$-norm blows-up. The main result that we prove is that there exists a unique constant $c_{0} \in \mathbb{R}$ such that the problem

$$
\begin{cases}-\Delta_{p} v+H(x, D v)+c_{0}=0 & \text { in } \Omega, \\ \lim _{x \rightarrow \partial \Omega} v(x)=+\infty & \text { on } \partial \Omega,\end{cases}
$$

admits a solution $v$. Such a constant $c_{0}$, which only depends on $p, \Omega$ and $H$, plays the role of an additive eigenvalue. Indeed we characterize this constant as follows:

$$
c_{0}=\sup \left\{c \in \mathbb{R}: \exists \varphi \in W^{1, \infty}(\Omega):-\Delta_{p} \varphi+H(x, D \varphi)+c \leq 0\right\} .
$$

Through the study of the ergodic limit of maximal solutions we are therefore back to the solvability of the Dirichlet problem (1.1) whenever $\lambda=0$. Indeed we prove (see Theorem 2.16) that the Dirichlet problem (1.1) with $\lambda=0$ admits a solution if $c_{0}>0$, while no solution exists if $c_{0}<0$.

Notice that in the special case $H(x, \xi)=\beta|\xi|^{p}-f(x)$ (for $\beta>0$ and $f$ smooth) the constant $c_{0}$ is a true eigenvalue; precisely, $c_{0}$ is the first eigenvalue and $w(x)=(p-1) e^{-\frac{\beta}{p-1} v(x)}$ is the first positive eigenfunction of the operator

$$
-\Delta_{p} u+\beta f(x)|u|^{p-2} u, \quad u \in W_{0}^{1, p}(\Omega)
$$

with Dirichlet boundary conditions.

The uniqueness (up to a constant) of the solution $v$ to 1.6 is a partially open question. If $p=2$ this is proved in 20 relying on the strong maximum principle. Unfortunately, the lack of a general strong comparison principle for the $p$-Laplace operator does not allow us to extend a similar uniqueness result for $p>2$. However, we provide a partial positive answer by using a strong comparison principle recently proved in [25, Theorem 1.4]. In particular, we prove that 1.6) has a unique solution $v$ (up to a constant) whenever $c_{0}+H(x, 0)>0$, a condition which is satisfied, in particular, if the oscillation of $H(x, 0)$ is not too large (see Corollary 2.18). 
The plan of the paper is the following. In the next section we collect the statements of all our main results: they are concerned with gradient bounds, the solvability of the Dirichlet problem, the existence and uniqueness of large solutions, and the study of the ergodic problem associated to those maximal solutions. The proofs of such results are left in the subsequent Sections. Even if the gradient estimates are preliminary to all results, this is the most technical part of the paper, so we decided to postpone to last sections the proofs of those estimates, together with some extension to Neumann boundary conditions.

Notation. Since $\Omega$ is assumed to be $C^{2}$, the distance function $\operatorname{dist}(x, \partial \Omega)$ is of class $C^{2}$ in a neighborhood of the boundary. For $\delta>0$, we set

$$
\Omega_{\delta}:=\{x \in \Omega: \operatorname{dist}(x, \partial \Omega)<\delta\} .
$$

In the sequel we denote by $d(x)$ a $C^{2}(\Omega)$ function which coincides with the distance near the boundary. Namely, there exists a $\underline{\delta}>0$ such that

$$
d(x) \equiv \operatorname{dist}(x, \partial \Omega) \quad \text { in } \Omega_{\underline{\delta}}
$$

We also recall that the outward unit normal satisfies $\nu=-D d$ at $\partial \Omega$.

\section{MAin Results OF THE PAPER}

In this Section we collect the main results of the paper, leaving the proofs for the subsequent sections.

2.1. Global gradient bounds. We start by exploiting the well known Bernstein method in order to get local and global gradient bounds for the solutions to $E_{\lambda}$. This method, which is by now classical, was originally introduced in [12] and extensively refined, in the case $p=2$, by J. Serrin and P.-L. Lions (see [28, [29, [30, [34, 35]), whereas weaker forms of this method to be used for viscosity solutions were later developed since [6]. For the $p$-Laplacian with $p \neq 2$, similar estimates have been derived by several authors for specific applications, like the study of long time behavior, see e.g. the recent paper 3 and the references therein. Our goal is to make a systematic study of those gradient bounds in terms of the structural growth of the first order terms, paying attention whether the estimates are depending or not on the $L^{\infty}$ bound of the solution.

Because of the generality of our structure conditions, we choose to use the classical version of Bernstein's method rather than some weak form which would be technically too much involved. This choice does not allow us to work directly on the solutions of the equation $\left(\sqrt{E_{\lambda}}\right)$, due to a lack of regularity, since solutions are in general no more regular than $C^{1, \alpha}(\bar{\Omega})$, for some $\alpha \in(0,1)$. So the strategy we use relies on a typical scheme of approximation. Namely, we consider the uniformly elliptic problem

$$
\lambda u_{\varepsilon}-\operatorname{div}\left(\left(\varepsilon+\left|D u_{\varepsilon}\right|^{2}\right)^{\frac{p-2}{2}} D u_{\varepsilon}\right)+H\left(x, D u_{\varepsilon}\right)=0 \quad \text { in } \Omega,
$$

with $\lambda \geq 0$ and $\varepsilon>0$. We aim at proving estimates independent of $\varepsilon$.

As far as the Hamiltonian term is concerned, first of all we suppose that

$$
H(x, \xi) \in C^{1}\left(\Omega \times \mathbb{R}^{N}\right) .
$$

Then, adapting to the $p$-Laplace operator the ideas developed by P.L. Lions ([29], [30]), we consider the following structure conditions:

there exist $\theta \in(0,1)$ and $\rho>0$ such that

$$
\liminf _{|\xi| \rightarrow+\infty}\left\{\rho|\xi|^{p+2}+\tau|\xi|^{2}\left[\xi \cdot H_{\xi}(x, \xi)-(p-1) H(x, \xi)\right]\right\}>0,
$$


and

$$
\liminf _{|\xi| \rightarrow+\infty} \frac{\xi \cdot H_{x}(x, \xi)+(1-\theta) \eta^{2}|\xi|^{2-p} H^{2}(x, \xi)}{\rho|\xi|^{p+2}+\tau|\xi|^{2}\left[\xi \cdot H_{\xi}(x, \xi)-(p-1) H(x, \xi)\right]} \geq 0,
$$

uniformly with respect to $x \in \Omega$, where $\eta=\frac{1}{|p-2|+\sqrt{N}}$ and $\tau$ is either 1 or -1 .

The meaning of the above hypothesis is that the term $\xi \cdot H_{x}(x, \xi)$ has to be dominated either by $|\xi|^{2-p} H^{2}(x, \xi)$ or, possibly, by $\rho|\xi|^{p+2}+\tau|\xi|^{2}\left[\xi \cdot H_{\xi}(x, \xi)-(p-1) H(x, \xi)\right]$. Notice that 2.3 . is meant to exploit the "(p-1)-convexity" (or concavity) of the Hamiltonian.

Let us remark that 2.4 can be rephrased in equivalent form as following: there exist $\theta \in(0,1)$ and $\rho, \delta_{0}>0$ such that for any $\delta \in\left(0, \delta_{0}\right)$ we have

$$
\begin{array}{ll}
\liminf _{|\xi| \rightarrow+\infty} & \xi \cdot H_{x}(x, \xi)+(1-\theta) \eta^{2}|\xi|^{2-p} H^{2}(x, \xi) \\
& +\delta\left\{\rho|\xi|^{p+2}+\tau|\xi|^{2}\left[\xi \cdot H_{\xi}(x, \xi)-(p-1) H(x, \xi)\right]\right\} \geq 0,
\end{array}
$$

uniformly with respect to $x \in \Omega$, where $\eta=\frac{1}{|p-2|+\sqrt{N}}$ and $\tau$ is either 1 or -1 .

It is easy to see that 2.3)-2.4 are equivalent to 2.3)-(2.5); moreover, up to replacing $\rho$ with a larger value, the above liminf can be as large as desired.

We also consider the following stronger condition, for the case where 2.3 does not hold: there exist $\theta \in(0,1)$ and $\rho, \delta_{0}>0$ such that for any $\delta \in\left(0, \delta_{0}\right)$ we have

$$
\begin{array}{ll}
\liminf _{|\xi| \rightarrow+\infty} & \xi \cdot H_{x}(x, \xi)+(1-\theta) \eta^{2}|\xi|^{2-p} H^{2}(x, \xi) \\
& +\delta\left\{\rho|\xi|^{p+2}-|\xi|^{2}\left|\xi \cdot H_{\xi}(x, \xi)-(p-1) H(x, \xi)\right|\right\} \geq 0,
\end{array}
$$

uniformly with respect to $x \in \Omega$, where $\eta=\frac{1}{|p-2|+\sqrt{N}}$.

Here we state our result concerning global estimates on the gradient. In this first result, the estimate for the gradient depends on the $L^{\infty}$ bound of the solution as well as on the gradient maximum at the boundary. This latter quantity will of course rely on the boundary conditions, and this issue will be discussed later on (so far we only discuss the solutions to the equation (2.1).

Theorem 2.1 (Global bound). Assume that $H(x, \xi)$ satisfies (2.2) and that (2.3)-(2.4) or (2.6) are satisfied. Then there exists a constant $c=c\left(\lambda, H,\left\|u_{\varepsilon}\right\|_{L^{\infty}(\Omega)}\right)$ such that any smooth solution to 2.1) satisfies

$$
\sup _{\bar{\Omega}}\left|D u_{\varepsilon}\right|^{2} \leq c+\sup _{\partial \Omega}\left|D u_{\varepsilon}\right|^{2} .
$$

Remark 2.2. The constant appearing in (2.7) depends on $H$ through all the quantities involved in the limits (2.3)-(2.4) or (2.6).

We stress that the Hamiltonian is not a priori restricted to have natural growth with respect to the gradient. However, the standard existence and regularity results for $u_{\varepsilon}$ hold true for lower order terms under the natural growth condition. In order to overcome this difficulty, it is enough to argue as follows.

Consider a family of Hamiltonians that approximate $H(x, \xi)$ in the following way:

$$
H_{n}(x, \xi)=H(x, \xi)-\varphi_{n}\left(H(x, \xi)-n|\xi|^{p}\right), \quad n \in \mathbb{N}
$$


where $\varphi_{n}(\cdot)$ is a smooth approximation of the function $\varphi(s)=s^{+}$. In particular, we may take $\varphi_{n}$ to be a convex function such that $\varphi_{n}(s) \equiv 0$ for $s \leq 0,0 \leq \varphi_{n}^{\prime}(s) \leq 1$ for any $s$ and $\varphi_{n}^{\prime \prime}(s) \equiv 0$ for $s>\frac{1}{n}$. Notice that $H_{n}$ has natural growth and is a smooth variation of the truncation function $\min (n, H(x, \xi))$.

It can be checked that, if $H$ satisfies the conditions (2.3)- (2.4) or (2.6), respectively, then $H_{n}$ satisfies the same conditions uniformly with respect to $n \in \mathbb{N}$. Actually this means that the parameters involved may be chosen independent of $n$ and the limit themselves are uniform with respect to $n \in \mathbb{N}$. Replacing $H$ with $H_{n}$, we are sure that solutions to $\left(E_{\lambda}\right.$ exist and are smooth, and the gradient bounds will be independent of $n$. This allows us to apply the estimates to Hamiltonians which possibly grow above the natural threshold of the power $|\xi|^{p}$.

The hypotheses involved in the above Theorem are quite general and cover a large class of nonlinear terms. Let us give some examples.

Example 1: Hamiltonians with natural growth.

If $H(x, \xi)$ satisfies, for some $c>0$,

$$
|H(x, \xi)|+|\xi|\left|H_{\xi}(x, \xi)\right| \leq c\left(|\xi|^{p}+1\right) \quad \forall \xi \in \mathbb{R}^{N} \text {, a.e. in } \Omega,
$$

then the term $\xi \cdot H_{\xi}(x, \xi)-(p-1) H(x, \xi)$ is always dominated by $\rho|\xi|^{p}$, for $\rho$ sufficiently large; in this case (2.3) is satisfied and (2.4) reduces to verify

$$
\liminf _{|\xi| \rightarrow+\infty} \frac{\xi \cdot H_{x}(x, \xi)+(1-\theta) \eta^{2}|\xi|^{2-p} H^{2}(x, \xi)}{|\xi|^{p+2}} \geq 0 \quad \text { uniformly in } \Omega,
$$

which is the case, for instance, if $\left|H_{x}(x, \xi)\right|=o\left(|\xi|^{p+1}\right)$ as $|\xi| \rightarrow \infty$, uniformly with respect to $x \in \Omega$.

Example 2: Convex hamiltonians.

Assume that $H(x, \xi)=h\left(|\xi|^{p-1}\right)-f(x)$ where $h$ is a positive increasing and convex function, at least for $|\xi|$ big enough, and that $f$ is smooth enough (say $W^{1, \infty}(\Omega)$ ).

Then the hypotheses of the above Theorem are easily fulfilled since the convexity of $h$ implies that

$$
\xi \cdot H_{\xi}(x, \xi)-(p-1) H(x, \xi)
$$

is always positive and it also dominates $|\xi \cdot D f|$, since $p>2$. So $(2.3)-(2.4)$ are clearly satisfied.

Observe that if $f=f(x, \xi)$ the same holds true if $|\xi||f(x, \xi)|+\left|f_{\xi}(x, \xi)\right|=O\left(|\xi|^{p}\right)$ and $\left|f_{x}(x, \xi)\right|=o\left(|\xi|^{p+1}\right)$, uniformly with respect to $x \in \Omega$.

Remark 2.3. Since for any $\varepsilon>0$ we have $|\xi|^{2}|H(x, \xi)| \leq \varepsilon|\xi|^{2-p} H^{2}(x, \xi)+C_{\varepsilon}|\xi|^{p+2}$, one can see that (2.6) is equivalent to ask

$$
\liminf _{|\xi| \rightarrow+\infty} \xi \cdot H_{x}(x, \xi)+(1-\theta) \eta^{2}|\xi|^{2-p} H^{2}(x, \xi)+\delta\left\{\rho|\xi|^{p+2}-|\xi|^{2}\left|\xi \cdot H_{\xi}(x, \xi)\right|\right\} \geq 0 .
$$

In particular, if $H(x, \xi)$ satisfies, for $|\xi|$ sufficiently large,

$$
\left|H_{\xi}(x, \xi) \cdot \xi\right| \leq c(1+|H(x, \xi)|)
$$

uniformly with respect to $x \in \Omega$, then it is enough to verify whether, for every $\delta$ sufficiently small,

$$
\liminf _{|\xi| \rightarrow+\infty} \xi \cdot H_{x}(x, \xi)+(1-\theta) \eta^{2}|\xi|^{2-p} H^{2}(x, \xi)+\delta \rho|\xi|^{p+2} \geq 0
$$

which is equivalent to (2.8), as in the case of natural growth Hamiltonian. 
Example 3: The role of $H^{2}(x, \xi)$.

There are cases in which the best contribution in the limits 2.5) or 2.6 is given by the term that involves $H^{2}$. Typically, in this case $H$ turns out to have a growth at infinity larger than $|\xi|^{p}$ and the term with $H^{2}$ may be relevant especially if (2.3) fails. Let us consider, for example, for $\alpha \in \mathbb{R}$,

$$
H(x, \xi)=|\xi|^{p-1}\left[|\xi|^{2}+\alpha \sin \left(|\xi|^{2}\right)\right]-f(x),
$$

with $f \in W^{1, \infty}(\Omega)$. By direct computations we notice that for any $\rho>0, \rho|\xi|^{p+2}+|\xi|^{2}[\xi$. $\left.H_{\xi}(x, \xi)-(p-1) H(x, \xi)\right]$ changes sign if $|\alpha|>1$ and so 2.3$)$ is not verified.

On the other hand $(2.6)$ holds true, since the term $H^{2}(\overline{x, \xi})|\xi|^{2-p}$ grows faster than $\rho|\xi|^{p+2}+$ $|\xi|^{2}\left[\xi \cdot H_{\xi}(x, \xi)-(p-1) H(x, \xi)\right]$ as $|\xi|$ diverges, for any value $\rho>0$ (uniformly with respect to $x \in \Omega$ ).

A slightly different version of such global gradient bounds are also proved in Section 7, with the straightforward application to the Neumann problem.

2.2. Interior gradient bounds. The second type of result that we prove is a local gradient bound, which holds thanks to the coercivity of the first order term. To be more precise, we assume that $H(x, \xi)$ is a $C^{1}$ function satisfying, for some locally bounded function $f$, for a.e. $x \in \Omega$ and for any $\xi \in \mathbb{R}^{N}$ with $|\xi|$ sufficiently large

$$
H(x, \xi) \geq h\left(|\xi|^{p-1}\right)-f(x)
$$

where

$$
h(s) \in C^{2}\left(\mathbb{R}^{+}\right) \quad \text { is an increasing and convex function such that } h(0)=0
$$

and the following growth hypotheses hold true:

$$
\int^{\infty} \frac{d \tau}{h(\tau)}<\infty \quad \text { and } \quad \limsup _{\tau \rightarrow \infty} \frac{\tau^{2} h^{\prime \prime}(\tau)}{h^{\prime}(\tau) \tau-h(\tau)}<\infty
$$

Notice that the integrability assumption in 2.11) implies that $h$ has a superlinear growth, namely $h(s) / s \rightarrow \infty$ as $s \rightarrow \infty$. As far as the second condition is concerned, this is just a technical assumption (that we need in the construction of suitable test functions), but it is anyway not very restrictive including all model examples with a regular behavior at infinity.

In addition to (2.9)-2.11), we also assume the following growth conditions: for any compact subset $\omega \subset \subset \Omega$, there exists a positive constant $c_{\omega}$ such that for a.e. $x \in \omega$ and for any $\xi \in \mathbb{R}^{N}$

$$
\left|H_{\xi}(x, \xi)\right||\xi| \leq c_{\omega}[H(x, \xi)+1]
$$

and

$$
\exists \theta \in(0,1): \quad H_{x}(x, \xi) \cdot \xi \leq(1-\theta) \eta^{2}|\xi|^{2-p} H^{2}(x, \xi)+c_{\omega} \quad \text { where } \quad \eta=\frac{1}{|p-2|+\sqrt{N}}
$$

Remark 2.4. Since, for any $\varepsilon>0$, we have

$$
|\xi| H(x, \xi) \leq \varepsilon|\xi|^{2-p} H^{2}(x, \xi)+c_{\varepsilon}|\xi|^{p},
$$

then assumption (2.13) holds whenever

$$
\left|H_{x}(x, \xi)\right| \leq c[H(x, \xi)+1] \quad \forall x \in \Omega, \xi \in \mathbb{R}^{N}
$$

and if $H(x, \xi) /|\xi|^{p-1}$ diverges (uniformly with respect to $x \in \Omega$ ) as $|\xi| \rightarrow \infty$. This latter fact is true, in particular, after (2.9)-2.11), in which case 2.14) gives a simple condition which implies 2.13).

Here we state the main result concerning local estimates. 
Theorem 2.5 (Local bound). Assume that $H(x, \xi)$ satisfies (2.2) and that (2.9)-2.13) are satisfied. Then for any $\omega \subset \subset \omega^{\prime} \subset \subset \Omega$ there exists $c=c\left(\omega^{\prime},\left\|\lambda u_{\varepsilon}\right\|_{L^{\infty}\left(\omega^{\prime}\right)},\|f\|_{L^{\infty}\left(\omega^{\prime}\right)}, H\right)$ such that any smooth solution to (2.1) satisfies

$$
\sup _{x \in \omega}\left|D u_{\varepsilon}\right| \leq c .
$$

Remark 2.6. Let us stress that the bound does not depend on $\left\|u_{\varepsilon}\right\|_{L^{\infty}}$ but only on $\left\|\lambda u_{\varepsilon}\right\|_{L^{\infty}}$. This is a major difference with the estimate of Theorem 2.1 and will be essentially used in the study of the ergodic problem.

2.3. The Dirichlet problem. We turn now the attention to the solvability of the Dirichlet problem (1.1). Let us first state a simple principle which is a consequence of Theorem 2.1. This extends a similar result for the case $p=2$ proved in [29].

Theorem 2.7. Assume that $H(x, \xi)$ satisfies (2.2) and (2.3)-(2.4) or (2.6) are satisfied, and that $H$ is bounded below. Then the Dirichlet problem 1.1) admits a solution $u \in W_{0}^{1, \infty}(\Omega)$ if (and only if) there exists a subsolution $\psi \in W_{0}^{1, \infty}(\Omega)$ of the same problem.

Of course it is interesting to understand in which cases one can provide subsolutions to (1.1). This is where the behavior of $H(x, \xi)$ at infinity plays a role, and two different regimes appear. In order to fix the ideas, we assume that, for a.e. $x \in \Omega$ and for any $\xi \in \mathbb{R}^{N}$ with $|\xi|$ sufficiently large, we have

$$
H(x, \xi) \leq h\left(|\xi|^{p-1}\right)+C
$$

where $C>0$ and $h(s) \in C^{0}\left(\mathbb{R}^{+}\right)$is an increasing function such that

$$
\int^{\infty} \frac{\tau^{\frac{1}{p-1}}}{h(\tau)} d \tau=\infty
$$

It is not difficult to realize that this condition guarantees the existence of barriers at the boundary and then it allows us to reduce the problem to the existence of a merely bounded sub solution.

Theorem 2.8. Assume that $H(x, \xi)$ satisfies (2.2) and (2.3)-(2.4) or (2.6) are satisfied, and that $H$ is bounded below. Assume in addition that (2.16)-(2.17) hold true. Then we have

(i) for any $\lambda>0$, the Dirichlet problem $\sqrt{1.1}$ admits a solution $u \in W_{0}^{1, \infty}(\Omega)$;

(ii) when $\lambda=0$, the Dirichlet problem 1.1) admits a solution $u \in W_{0}^{1, \infty}(\Omega)$ if and only if there exists a bounded subsolution $\psi$ of $E_{\lambda}$.

Let us stress that the conclusions of the above result would be false if condition $(2.17)$ is not satisfied, unless the constant $C$ in 2.16 is sufficiently small (see also Remark 3.1).

In fact, in the model example $H(x, D u)=h\left(|D u|^{p-1}\right)-f(x)$, whenever $f \geq 0$ the problem has always a Lipschitz solution. But in general, a result as in Theorem 2.8 cannot hold if

$$
\int^{\infty} \frac{\tau^{\frac{1}{p-1}}}{h(\tau)} d \tau<\infty
$$

unless $\left\|f^{-}\right\|_{L^{\infty}(\Omega)}$ is sufficiently small. Indeed, if 2.18 holds true, there exists a maximal solution $U$ satisfying

$$
\left\{\begin{array}{l}
\lambda U-\Delta_{p} U+H(x, D U)=0 \\
U \text { is the maximal solution in } \Omega, U \in L^{\infty}(\Omega) .
\end{array} \text { in } \Omega,\right.
$$

Such a bounded maximal solution, which is the solution of the associated state constraint problem, is Lipschitz inside $\Omega$ but its gradient blows-up at the boundary. In particular, if $\Omega$ is a ball 
and $H(\cdot, \xi)$ is radial, then $U$ is radial (hence the boundary datum is a maximal constant) and, near the boundary, we have $|\nabla U(x)|^{p-1} \sim \Phi^{-1}(d(x))$ where $\Phi(\xi)=\int_{\xi}^{\infty} \frac{d s}{h(s)}$. Notice that the primitive of $\left[\Phi^{-1}\right]^{\frac{1}{p-1}}$ is bounded if and only if 2.18 holds true.

For a study of maximal solutions under condition (2.18), we refer to [7] for a general discussion, or to [5] for the model case of $p$-Laplacian with $H(x, D u)=|D u|^{q}$, with $q>p$.

We give an application of the above results in the following corollary, which is concerned with a simple model problem. For the case $p=2$, next result was previously proved in [2].

Corollary 2.9. Consider the Dirichlet problem

$$
\begin{cases}\lambda u-\Delta_{p} u+h\left(|D u|^{p-1}\right)=\mu f(x) & \text { in } \Omega, \\ u=0 & \text { on } \partial \Omega,\end{cases}
$$

where $\lambda, \mu \in \mathbb{R}^{+}, h(s) \in C^{1}\left(\mathbb{R}^{+}\right)$is an increasing and convex function and $f \in W^{1, \infty}(\Omega), f \leq 0$, with $f\left(x_{0}\right)<0$ for at least some $x_{0} \in \partial \Omega$.

Then:

(i) if (2.17) is satisfied, for any $\lambda>0$ the Dirichlet problem (2.19) admits a solution $u \in$ $W_{0}^{1, \infty}(\Omega)$.

(ii) if (2.18) is satisfied or if $\lambda=0$, there exists $\mu_{0}>0$ such that the Dirichlet problem (2.19) admits a solution $u \in W_{0}^{1, \infty}(\Omega)$ if $\mu<\mu_{0}$, while no solutions exist if $\mu>\mu_{0}$.

2.4. Large solutions. Here we study the maximal solutions associated to $\left(E_{\lambda}\right)$, under suitable growth assumptions on $H(x, \xi)$. We focus our attention on the case $H(x, \xi)$ has natural growth, in which case the maximal solutions blow-up at the boundary. In literature such solutions are usually called large solutions.

We start by giving a weak notion of solution to

$$
\left\{\begin{array}{l}
\lambda u-\Delta_{p} u+H(x, D u)=0 \quad \text { in } \Omega, \\
\lim _{x \rightarrow \partial \Omega} u(x)=+\infty
\end{array}\right.
$$

To this purpose, we use the standard notion of weak solution inside $\Omega$, while the boundary blowup condition can be defined using truncations as in 21. In the sequel, for any positive $k$ we define $T_{k}(s)$ the following continuous function

$$
T_{k}(s)=\max \{-k, \min \{s, k\}\} .
$$

We denote by $W_{\mathrm{c}}^{1, p}(\Omega)$ the space of $W^{1, p}(\Omega)$ function with compact support in $\Omega$ (precisely, functions $\psi$ which belong to $W_{0}^{1, p}(\omega)$ for some smooth $\omega \subset \subset \Omega$, and extended to zero outside $\omega)$.

Definition 2.10. We say that a function $u \in W_{\mathrm{loc}}^{1, p}(\Omega) \cap L_{\mathrm{loc}}^{\infty}(\Omega)$ is a subsolution (supersolution, respectively) for $\left.E_{\lambda}\right)$ if $H(x, D u) \in L_{\mathrm{loc}}^{1}(\Omega)$ and

$$
\begin{gathered}
\int_{\Omega} \lambda u \psi+\int_{\Omega}|D u|^{p-2} D u \cdot D \psi+\int_{\Omega} H(x, D u) \psi \leq(\geq) 0, \\
\forall \psi \in W_{c}^{1, p}(\Omega) \cap L^{\infty}(\Omega), \quad \psi \geq 0 .
\end{gathered}
$$

Consequently, $u$ is a solution for $E_{\lambda}$ if it is both a subsolution and a supersolution.

$A$ weak solution to $\left(P_{\lambda}\right)$ is a function which is a solution to $\left(E_{\lambda}\right)$, is bounded below and satisfies

$$
k-T_{k}(u) \in W_{0}^{1, p}(\Omega), \quad \forall k \geq 0 .
$$


As far as the Hamiltonian $H(x, \xi)$ is concerned, we deal with the case in which $H(x, \xi)$ behaves, at least near the boundary, as a power of $|\xi|$.

More precisely, we assume that there exist $\beta>0$ and $q \in(p-1, p]$ such that

$$
\forall \varepsilon>0 \quad \exists \delta>0:\left.\quad|H(x, \xi)-\beta| \xi\right|^{q} \mid \leq \varepsilon\left[|\xi|^{q}+d^{-\frac{q}{q-(p-1)}}(x)\right] \quad \forall x \in \Omega_{\delta}, \forall \xi \in \mathbb{R}^{N} .
$$

Moreover, we assume a natural growth condition throughout all the domain, namely that

$$
f(x)-\gamma_{1}|\xi|^{p} \leq H(x, \xi) \leq \gamma\left(|\xi|^{p}+1\right) \quad \forall x \in \Omega, \forall \xi \in \mathbb{R}^{N}
$$

for some $\gamma, \gamma_{1}>0$, and a function $f(x)$ such that

$$
f \in L_{\mathrm{loc}}^{m}(\Omega) \text { with } m>\frac{N}{p} .
$$

Notice that the growth conditions on $H(x, \xi)$ are asymmetric; some global condition is required from above in order to control the lower bound of solutions, while the upper bound is locally controlled thanks to the coercive condition 2.22 which implies a barrier effect near the boundary.

In order to have uniqueness of the large solution, we also add some hypotheses which allow us to compare the solutions near the boundary and to apply, locally, the comparison principle between bounded $W^{1, p}$ solutions of $E_{\lambda}$.

We give two technical conditions which are sufficient for uniqueness to hold. In one case, we assume some extra regularity on the Hamiltonian term, namely that $H$ is $C^{1}$ with respect to $\xi$ and satisfies

$$
\forall \omega \subset \subset \Omega \quad \exists c_{\omega}: \quad\left|H_{\xi}(x, \xi)\right| \leq c_{\omega}\left(1+|\xi|^{p-1}\right) \quad \forall \xi \in \mathbb{R}^{N}, \forall x \in \omega .
$$

Observe that such a condition is not very restrictive, since we already are in a natural growth framework. In addition, we assume that, for any $\varepsilon$ sufficiently small, $H$ satisfies

$$
H(x,(1-\varepsilon) \xi)-(1-\varepsilon)^{p-1} H(x, \xi) \leq-\varepsilon \rho(x)|\xi|^{q}+\varepsilon \sigma(x) \quad \forall x \in \Omega, \forall \xi \in \mathbb{R}^{N}
$$

for some function $\rho(x), \sigma(x) \in L_{\mathrm{loc}}^{\infty}(\Omega)$ such that

$$
\lim _{d(x) \rightarrow 0} d^{\frac{q}{q-(p-1)}} \sigma(x)=0, \quad \rho(x) \geq 0 \quad \text { and } \exists \delta_{0}>0: \quad \rho(x) \geq \rho>0 \quad \forall x \in \Omega_{\delta_{0}} .
$$

Alternatively to $2.25-2.26$, we might assume that a global convexity-type condition hold in the whole domain. Precisely, we may assume that, for any $\varepsilon$ sufficiently small, there exists a constant $c_{\varepsilon}>0$ such that, for all $x \in \Omega$ and $\xi, \varsigma \in \mathbb{R}^{N}$,

$$
H(x, \xi)-(1-\varepsilon)^{p-1} H\left(x, \frac{\varsigma}{1-\varepsilon}\right) \leq-\varepsilon \rho(x)|\varsigma|^{q}+\varepsilon \sigma(x)+c_{\varepsilon}\left[|\xi-\varsigma|^{p-1}(1+|\xi-\varsigma|)\right]
$$

$$
\begin{aligned}
& \text { for some function } \rho(x), \sigma(x) \in L_{\mathrm{loc}}^{\infty}(\Omega) \text { such that } \\
& \lim _{d(x) \rightarrow 0} d^{\frac{q}{q-(p-1)}} \sigma(x)=0, \quad \rho(x) \geq 0 \quad \text { and } \exists \delta_{0}>0: \quad \rho(x) \geq \rho>0 \quad \forall x \in \Omega_{\delta_{0}} .
\end{aligned}
$$

Observe that 2.27 implies 2.26 by choosing $\zeta=\xi$. So, the main difference between the two set of conditions is that 2.27) avoids an explicit requirement upon $H_{\xi}$. Let us also stress that, if solutions are locally Lipschitz, then condition 2.26) can be asked to hold only in a neighborhood of the boundary.

Theorem 2.11. Assume that $\Omega$ is a bounded $C^{2}$ domain in $\mathbb{R}^{N}, N \geq 2$. Let $\lambda>0$ and suppose

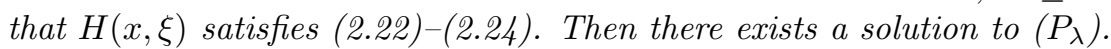

In addition, suppose that either (2.25)-(2.26) or (2.27) hold true. Then the solution is unique. 
Remark 2.12. Let us point out some situations for which the above hypotheses hold. The easiest example happens if we look at the function $H$ as a power nonlinearity plus some perturbation. To be more precise, let us define $\Gamma(x, \xi)=H(x, \xi)-\beta|\xi|^{q}$ with $\beta>0$ and $q \in(p-1, p]$. Then, (2.26) is fulfilled if we assume that

$$
\Gamma(x, \xi(1-\varepsilon))-(1-\varepsilon)^{p-1} \Gamma(x, \xi) \leq \varepsilon h(x, \xi)
$$

where $h \in C^{0}: \Omega \times \mathbb{R}^{N} \rightarrow \mathbb{R}^{+}$is such that

$$
\begin{aligned}
& \forall \eta>0 \quad \exists R>0: h(x, \xi) \leq \eta|\xi|^{q}+\sigma(x) \\
& \forall x \in \Omega \forall \xi \in \mathbb{R}^{N}:|\xi| \geq R \quad \text { where } \quad \sigma(x) \in L_{\mathrm{loc}}^{\infty}(\Omega): \quad \lim _{d \rightarrow 0} \sigma(x) d^{\frac{q}{q-(p-1)}}=0
\end{aligned}
$$

In particular, this happens if (2.22) holds and $\Gamma(x, \cdot)$ is a differentiable function with $\left|\Gamma_{\xi}(x, \xi)\right| \leq$ $\tilde{h}(x, \xi)$ for some continuous function $\tilde{h}$ satisfying

$$
\begin{gathered}
\forall \eta>0 \quad \exists \delta>0: \quad|\tilde{h}(x, \xi)| \leq \eta\left[|\xi|^{q-1}+d^{\tilde{\gamma}}(x)\right] \\
\forall x \in \Omega_{\delta} \forall \xi \in \mathbb{R}^{N}:|\xi| \geq R \quad \text { where } \quad \tilde{\gamma}=\frac{q-1}{q-(p-1)} .
\end{gathered}
$$

Remark 2.13. Observe that, by definition, the large solutions belong to $L_{\mathrm{loc}}^{\infty}(\Omega)$. Anyway, the same uniqueness result holds true even if we deal with weak solutions that are not necessarily locally bounded, just by considering a suitable modification on the global behavior of the hamiltonian term, namely replacing (2.23) with

$$
f(x)-\gamma_{1}|\xi|^{p-1} \leq H(x, \xi) \leq \gamma\left(|\xi|^{p}+1\right) \quad \forall x \in \Omega, \forall \xi \in \mathbb{R}^{N}, \text { for some } \gamma, \gamma_{1}>0 .
$$

and $f(x)$ as in 2.24). Such a modification would be necessary since otherwise some counterexample would exist to uniqueness of weak unbounded solutions (see [1]).

Remark 2.14. Let us stress that the existence of large solutions would not need the function $H$ to have a precise asymptotic behavior as $|\xi| \rightarrow \infty$, in particular 2.22) could be replaced by

$$
\beta_{1}|\xi|^{q}-\sigma(x) \leq H(x, \xi) \leq \beta_{2}|\xi|^{q}+\sigma(x)
$$

with possibly different $\beta_{1}, \beta_{2}>0$ and $\sigma$ such that $\sigma(x) d^{\frac{q}{q-(p-1)}}(x) \rightarrow 0$ as $d(x) \rightarrow 0$.

2.5. The ergodic problem. We study now the behavior of the solutions of $\left(P_{\lambda}\right)$ as $\lambda \rightarrow 0$. This is also called the ergodic limit since, when $p=2$, it is related to ergodicity properties of the underlying controlled diffusion process, see [20. As shown in [10, [32, this singular limit is also strongly connected with both the solvability of the Dirichlet problem (1.1) and the long time behavior of the associated time-dependent problem.

In order to study the limit of solutions to $\left(P_{\lambda}\right)$ as $\lambda \rightarrow 0$, we reinforce some conditions on the function $H(x, s, \xi)$ in order to make use of the local gradient estimates of Theorem 2.5. In particular, we need a coercivity of the nonlinear term with respect to the gradient, so we suppose that hypotheses 2.9-2.11) are in force. Moreover, we assume that $H(x, \xi) \in C^{1}\left(\Omega \times \mathbb{R}^{N}\right)$ and satisfies:

$$
\forall \omega \subset \subset \Omega \exists c_{\omega}>0: \quad\left[\left|H_{x}(x, \xi)\right|+\left|H_{\xi}(x, \xi)\right||\xi|\right] \leq c_{\omega}(1+H(x, \xi)) \quad \forall x \in \omega, \xi \in \mathbb{R}^{N},
$$

which ensures that conditions 2.12 -2.13 are satisfied (see Remark 2.4). Finally we assume that $\Omega$ is connected, which is customary in this kind of results.

Here is our main result concerning the ergodic problem. 
Theorem 2.15. Assume that $\Omega$ is a $C^{2}$ bounded and connected domain in $\mathbb{R}^{N}, N \geq 2$. Assume that conditions (2.9)-(2.11), (2.22)-(2.24) and (2.26) are satisfied, and, in addition, that (2.30) holds true. Let $u_{\lambda}$ be the unique solution to $\left(P_{\lambda}\right)$. Then:

(i) There exists a unique $c_{0} \in \mathbb{R}$ such that the problem

$$
\begin{cases}-\Delta_{p} v+H(x, D v)+c_{0}=0 & \text { in } \Omega, \\ v=+\infty & \text { on } \partial \Omega,\end{cases}
$$

admits a solution $v$.

(ii) We have

$$
\lim _{\lambda \rightarrow 0} \lambda u_{\lambda}(x)=c_{0} \quad \text { locally uniformly in } \Omega .
$$

(iii) Given $x_{0} \in \Omega$ and setting $v_{\lambda}(x)=u_{\lambda}(x)-u_{\lambda}\left(x_{0}\right)$, we have, for a subsequence (not relabeled)

$$
\lim _{\lambda \rightarrow 0} v_{\lambda}(x)=v(x) \quad \text { locally uniformly in } \Omega,
$$

where $v(x) \in C^{1, \alpha}(\Omega)$ for some $\alpha \in(0,1)$ is a solution to 2.31).

The constant $c_{0}$ given by Theorem 2.15 plays a crucial role in the solvability of the Dirichlet problem (1.1) when $\lambda=0$, as pointed out in [32] for the case $p=2$. Indeed, $c_{0}$ enjoys the following characterization which somehow justifies the terminology of nonlinear additive eigenvalue which is often used for this constant. We recall that, in the case $p=2$, further properties of $c_{0}$ shared with the first eigenvalue, like for instance the Faber-Krahn property, are exploited in [17].

Theorem 2.16. Under the hypothesis of Theorem 2.15, we have that

$$
\begin{aligned}
c_{0} & =\sup \left\{c \in \mathbb{R}: \exists \varphi \in W^{1, p}(\Omega) \cap L^{\infty}(\Omega):-\Delta_{p} \varphi+H(x, D \varphi)+c \leq 0\right\} \\
& =\sup \left\{c \in \mathbb{R}: \exists \varphi \in W^{1, \infty}(\Omega):-\Delta_{p} \varphi+H(x, D \varphi)+c \leq 0\right\} .
\end{aligned}
$$

In particular, the Dirichlet problem

$$
\begin{cases}-\Delta_{p} u+H(x, D u)=0 & \text { in } \Omega, \\ u=0 & \text { on } \partial \Omega,\end{cases}
$$

admits a solution whenever $c_{0}>0$, and does not possess any solution whenever $c_{0}<0$.

The uniqueness (up to a constant) of the solution $v$ found in Theorem 2.15 is a delicate and interesting problem. In the case $p=2$ this is always true as a consequence of the strong maximum principle. Even for quasilinear operators which are uniformly elliptic, the uniqueness can be proved; e.g. for the problem (2.1) the uniqueness holds at least when $H$ is a power (see [33). In the degenerate case, the question is much more delicate because it is related to the validity of a strong comparison principle. We use here a recent result of this kind proved in 25, Theorem 1.4], which requires an extra condition related to the degeneracy of the Hamiltonian, namely that

$$
\left|H_{\xi}(x, \xi)\right| \leq c|\xi|^{\frac{p-2}{2}} \quad \forall \xi \in \mathbb{R}^{N}:|\xi| \leq 1 \quad \text { for a.e. } x \in \Omega
$$

Then we give a partial positive answer by proving that uniqueness of solutions to 2.31) holds under the extra condition $H(x, 0)+c_{0}>0$. Notice that this is an implicit condition (since $c_{0}$ depends itself on $H(x, 0))$, but next we will discuss more explicit conditions under which our result applies. 
Theorem 2.17. In addition to the assumptions of Theorem 2.15, assume that 2.35) holds true. Let $c_{0}$ be the unique constant given by Theorem 2.15; if we have $H(x, 0)+c_{0}>0$ in $\Omega$, then the solution $v$ to problem (2.31) is unique up to a constant.

We now exploit the condition $H(x, 0)+c_{0}>0$ thanks to the characterization of $c_{0}$ given by Theorem 2.16. Eventually, we can derive sufficient conditions for the uniqueness (up to a constant) of solutions to 2.31, which are given in terms of the Dirichlet problem

$$
\begin{cases}-\Delta_{p} u+H(x, D u)=\inf _{\Omega} H(x, 0) & \text { in } \Omega, \\ u=0 & \text { on } \partial \Omega,\end{cases}
$$

or, otherwise, in terms of the oscillation of $H(x, 0)$.

Corollary 2.18. Suppose that the assumptions of Theorem 2.15 and 2.35) hold true. Assume that the Dirichlet problem (2.36) admits a strict subsolution, i.e. there exists $c>-\inf H(x, 0)$ such that the problem

$$
\begin{cases}-\Delta_{p} \psi+H(x, D \psi)+c=0 & \text { in } \Omega, \\ \psi=0 & \text { on } \partial \Omega,\end{cases}
$$

admits a solution $\psi$. Then, the solution $v$ to problem (2.31) is unique up to a constant.

In particular, there exists a constant $K_{0}>0$ such that whenever

$$
\sup _{\Omega} H(x, 0)-\inf _{\Omega} H(x, 0)<K_{0}
$$

then the solution $v$ to problem (2.31) is unique up to a constant.

\section{Proof of the Results on the Dirichlet problem}

In this section we collect the proof of the main results about the Dirichlet problem (1.1).

\section{Proof of Theorem 2.7.}

We only need to prove that the existence of a $W_{0}^{1, \infty}(\Omega)$ subsolution $\psi$ implies the existence of a solution.

Consider the solution $u_{\varepsilon}$ of equation $(6.9)$, and assume that $\lambda>0$. If we replace $H(x, \xi)$ with $H_{n}(x, \xi)=\min \left(H(x, \xi), n|\xi|^{p}\right)$, then the existence of $u_{\varepsilon}$ is guaranteed by the natural growth of the Hamiltonian. However, since we want to apply Theorem 2.1 we need $H_{n}$ to be $C^{1}$. So, as explained in Remark 2.2 we actually take $H_{n}=H(x, \xi)-\varphi_{n}\left(H(x, \xi)-n|\xi|^{p}\right)$ where $\varphi$ is a $C^{1}$ function such that $0 \leq \varphi^{\prime}(s) \leq 1, \varphi(s) \equiv 0$ for $s \leq 0$ and $\varphi^{\prime}(s) \equiv 1$ for $s \geq \frac{1}{n}$. We stress that $H_{n}$ still satisfies (2.3)-(2.4) or (2.6) uniformly with respect to $n$.

Since $H_{n}(x, \xi)$ has natural growth, for fixed $n$ we have that $u_{\varepsilon}$ converges uniformly to the unique solution $u_{n} \in W_{0}^{1, p}(\Omega) \cap L^{\infty}(\Omega)$ of

$$
\lambda u_{n}-\Delta_{p} u_{n}+H_{n}\left(x, D u_{n}\right)=0 \quad \text { in } \Omega,
$$

and, by classical results on $p$-Laplace type equations, $\left\{u_{\varepsilon}\right\}_{\varepsilon}$ is bounded in $C^{1, \alpha}(\bar{\Omega})$ and therefore compact in $C^{1}(\bar{\Omega})$. This means that the gradient estimates obtained on $u_{\varepsilon}$ hold for $u_{n}$ in the limit. Hence, from Theorem 2.1 we deduce that

$$
\sup _{\bar{\Omega}}\left|D u_{n}\right|^{2} \leq c+\sup _{\partial \Omega}\left|D u_{n}\right|^{2}
$$

for some constant $c$ independent of $n$. 
Let now $U \in W_{0}^{1, p}(\Omega) \cap L^{\infty}(\Omega)$ be the solution of

$$
\begin{cases}\lambda U-\Delta_{p} U+h_{0}=0 & \text { in } \Omega, \\ U=0 & \text { on } \partial \Omega,\end{cases}
$$

where $h_{0} \in \mathbb{R}$ is a bound from below for $H(x, \xi)$. Observe that, once again thanks to the standard regularity of the $p$-Laplace equation, $U \in C^{1, \alpha}(\bar{\Omega})$, for some $\alpha \in(0,1)$.

Since, by comparison (see e.g. Theorem 1.2 in [25]), we have $\psi \leq u_{n} \leq U$, this means that $\left\|u_{n}\right\|_{L^{\infty}(\Omega)}$ is uniformly bounded. In addition, using the Dirichlet condition, in a neighborhood of the boundary $\left|u_{n}\right| \leq k d(x)$ for some $k>0$, where $d(x)$ is the distance function. So we also deduce that $\left\|D u_{n}\right\|_{L^{\infty}(\partial \Omega)}$ is uniformly bounded. Thus we conclude from (3.1) that $D u_{n}$ is bounded in $\Omega$, and passing to the limit with respect to $n$ we find a Lipschitz solution to (1.1).

In the case $\lambda=0$, the same reasoning can be applied to construct a solution to

$$
\varepsilon u-\Delta_{p} u+H(x, D u)=\varepsilon\|\psi\|_{L^{\infty}(\Omega)} \quad \text { in } \Omega,
$$

with a Lipschitz norm which is uniformly bounded with respect to $\varepsilon$. Passing to the limit yields a solution with $\lambda=0$.

\section{Proof of Theorem 2.8.}

Thanks to (2.16), when $\lambda>0$ a subsolution is given by

$$
\psi(x)=\max \left(-\varphi_{k}(d(x)),-\frac{C}{\lambda}\right)
$$

where $d(x)$ is the distance function and $\varphi_{k}$ is the solution of the 1-d problem

$$
\left\{\begin{array}{l}
-(p-1) \varphi_{k}^{\prime \prime}\left|\varphi_{k}^{\prime}\right|^{p-2}=2 h\left(\left|\varphi_{k}^{\prime}\right|^{p-1}\right) \quad \text { in }\left(0, \delta_{0}\right), \\
\varphi_{k}^{\prime}(0)=k, \\
\varphi_{k}(0)=0,
\end{array}\right.
$$

where $\delta_{0}$ is sufficiently small so that $d(x)$ is smooth and $|\nabla d(x)|=1$ whenever $d(x) \leq \delta_{0}$.

We claim that choosing $k$ sufficiently large, it turns out that $-\varphi_{k}(d(x))$ is a subsolution in the neighborhood $\left\{x: d(x)<\delta_{0}\right\}$, and in addition $-\varphi_{k}\left(\delta_{0}\right)<-\frac{\|f\|_{\infty}}{\lambda}$, so that $\psi$ is a subsolution as well. Indeed, by setting

we have

$$
H_{k}(s):=\frac{p-1}{2} \int_{s}^{k} \frac{\tau^{p-2}}{h\left(\tau^{p-1}\right)} d \tau
$$

$$
\varphi_{k}\left(\delta_{0}\right)=\int_{0}^{\delta_{0}} H_{k}^{-1}(\xi) d \xi=\int_{H_{k}^{-1}\left(\delta_{0}\right)}^{k} \frac{\tau^{p-1}}{h\left(\tau^{p-1}\right)} d \tau .
$$

There is no loss of generality if we suppose that $h$ is super linear and $\int^{\infty} \frac{1}{h(\tau)} d \tau<\infty$, since we may always replace $h$ with $\max \left(h(s), s^{p^{\prime}}\right)$. This means that $H_{\infty}(s)$ is well defined and $H_{k}(s)$ converges to $H_{\infty}(s)$ as $k \rightarrow \infty$. Therefore, on account of (2.17), we have

$$
\lim _{k \rightarrow \infty} \int_{H_{k}^{-1}\left(\delta_{0}\right)}^{k} \frac{\tau^{p-1}}{h\left(\tau^{p-1}\right)} d \tau=\infty
$$

So by choosing $k$ sufficiently large we have $\varphi_{k}\left(\delta_{0}\right)>\frac{\|f\|_{\infty}}{\lambda}$. Moreover, since $\left|\varphi_{k}^{\prime}\right|^{p-1}=o\left(h\left(\left|\varphi_{k}^{\prime}\right|^{p-1}\right)\right)$ whenever $\left|\varphi_{k}^{\prime}\right| \rightarrow \infty$, for $k$ sufficiently large we also have

$$
\Delta_{p} \varphi_{k}(d(x))=(p-1) \varphi_{k}^{\prime \prime}\left|\varphi_{k}^{\prime}\right|^{p-2}+o\left(h\left(\left|\varphi_{k}^{\prime}\right|^{p-1}\right)\right)
$$

and we can choose $k$ so that

$$
\lambda \varphi_{k}(d(x))-\Delta_{p} \varphi_{k}(d(x)) \geq h\left(\left|\varphi_{k}^{\prime}\right|^{p-1}\right)+C \geq H\left(x,-D \varphi_{k}(d(x))\right) .
$$


Therefore, $\psi$ is a $W_{0}^{1, \infty}(\Omega)$ sub solution. By Theorem 2.7, (i) is proved.

As far as (ii) is concerned, one should observe that the existence of a bounded subsolution implies that the sequence $\left\{u_{\varepsilon}\right\}$ constructed in Theorem 2.7 is uniformly bounded; moreover, the construction of a local barrier near the boundary (actually, a strict subsolution), together with the comparison principle, imply that $\left|D u_{n}\right|$ is uniformly bounded at the boundary. Therefore, applying the estimates of Theorem 2.1 we conclude as in Theorem 2.7 that $u_{n}$ is bounded in Lipschitz norm and its limit is a $W_{0}^{1, \infty}(\Omega)$ solution of $(1.1)$.

Remark 3.1. Notice that condition (2.17) was needed in the above proof in order to deduce that $\varphi_{k}$ could be arbitrary large as $k \rightarrow \infty$. If (2.17) were violated, then the function $\varphi_{k}$ is uniformly bounded with respect to $k$, and so the existence of a subsolution would be guaranteed only if $C$ is sufficiently small.

We conclude with the proof of Corollary 2.9.

\section{Proof of Corollary 2.9 ,}

Statement (i) is a consequence of Theorem 2.8 Let us prove (ii).

We start by considering the case $\lambda=0$; we first prove that for $\mu$ large enough, there exists no solution to 2.19.

We first observe that, by assumption there exists at least a ball $B_{r} \subset \subset \Omega$ such that $f<0$ in $B_{r}$. Let $\varphi$ be a positive $C_{c}^{2}(\Omega)$ function supported on $\omega \subset \subset \Omega$ with $B_{r} \subset \omega$ and such that $\varphi \equiv 1$ over $B_{r}$. Using $\varphi$ as test function in 2.19 , after the integration by parts we deduce:

$$
\int_{\Omega} h\left(|D u|^{p-1}\right) \varphi \leq \int_{\Omega} \mu f(x) \varphi+\int_{\Omega}|D u|^{p-1}|D \varphi| .
$$

Using that $h$ is convex, we apply the generalized Young inequality, i.e.

$$
\forall a, b \in \mathbb{R} \quad a b \leq h(|a|)+h^{*}(|b|) \quad \text { where } \quad h^{*}(y):=\sup _{r \in \mathbb{R}}[y r-h(r)],
$$

we deduce that

$$
-\mu \int_{\Omega} f(x) \varphi \leq \int_{\Omega} \varphi h^{*}\left(\frac{|D \varphi|}{\varphi}\right)
$$

and the integral in the right hand side is bounded by a constant that only depends on $\varphi$ (see Lemma 6.4 for more details).

Thus we get

$$
\mu c_{f} \leq c_{\varphi} \quad \text { where } c_{f}=\inf _{B_{r}}|f(x)|>0 .
$$

Consequently for $\mu$ sufficiently large we get a contradiction.

Assume now that $\lambda>0$ and $(2.18)$ holds true. By assumption, there exists $x_{0} \in \partial \Omega$ such that $f\left(x_{0}\right)<0$; since $\Omega$ is smooth (in particular, it satisfies the inner sphere condition), there exists a ball $B_{r} \subset \Omega$, which is tangential at $x_{0} \in \partial \Omega$, such that $u$ satisfies

$$
\lambda u-\Delta_{p} u+h\left(|D u|^{p-1}\right) \leq-\mu c_{0} \quad \text { in } B_{r},
$$

for some $c_{0}>0$. Therefore $u \leq U$, where $U$ is the radial solution of

$$
\left\{\begin{array}{l}
\lambda U-\Delta_{p} U+h\left(|D U|^{p-1}\right)=-\mu c_{0} \quad \text { in } B_{r} \\
U(r)=0
\end{array}\right.
$$


However, one can easily check in the radial case that there exists a maximal value $\mu^{*}$ such that at $\mu=\mu^{*}$ we have $U^{\prime}(r)=\infty$, namely $U^{\prime}(r)$ tends to $\infty$ as $\mu \uparrow \mu^{*}$. Since by comparison we have $\frac{\partial u}{\partial \nu}\left(x_{0}\right) \geq U^{\prime}(r)$, we reach a contradiction with the Lipschitz character of $u$.

We are left to prove that $E=\left\{\mu \in \mathbb{R}^{+}: 2.19\right.$ has a Lipschitz solution $\}$ is a non empty interval. We first observe that, if $\mu$ is sufficiently small, a $W_{0}^{1, \infty}$ sub solution can be constructed as in Theorem 2.8 (see also Remark 3.1), then a solution exists due to Theorem 2.7. Finally, notice that a solution to 2.19 corresponding to $\mu$ turns out to be a subsolution to the same problem for any $\mu^{\prime}<\mu$; hence invoking Theorem 2.7 there exists a Lipschitz solution of (2.19) for $\mu^{\prime}$ too. This means that $E$ is an interval and a solution exists for all $\mu \in\left[0, \mu_{0}\right)$, where $\mu_{0}=\sup E<\infty$.

Observe that for $\lambda>0$ (see [25], Theorem 2.3) uniqueness of Lipschitz solutions hold.

\section{Proof of the Results on large solutions}

This Section is devoted to the proof of Theorem 2.11. concerning problem $\left(P_{\lambda}\right)$.

4.1. Existence. The proof of the existence of solutions follows by a typical approximation procedure. Thanks to 2.22, the Hamiltonian is coercive near the boundary in a way to prevent the propagation of singularity; indeed, a local uniform bound exists independently from the boundary values. Once a local bound is established, we use standard local compactness to deduce the existence of a solution.

\section{Proof of Theorem 2.11; Existence}

The existence is obtained by a standard approximation. In order to desingularize the equation at $\partial \Omega$, we consider a sequence of solutions to the following problem:

$$
\begin{cases}\lambda u_{n}-\Delta_{p} u_{n}+H\left(x, D u_{n}\right)=0 & \text { in } \Omega^{n} \\ u_{n}=n & \text { on } \partial \Omega^{n},\end{cases}
$$

where $\Omega^{n}=\left\{x \in \Omega: d(x, \partial \Omega)>\frac{1}{n}\right\}$, for $n \geq n_{0}$ for a certain $n_{0} \in \mathbb{N}$.

The existence of a sequence of smooth solutions $u_{n}$ (say $C^{1, \alpha}\left(\Omega^{n}\right) \cap C^{0}\left(\overline{\Omega^{n}}\right), \alpha \in(0,1)$ ) is a consequence of [18, Theorem 15.8]. Moreover, by (2.23), $u_{n}$ satisfies

$$
\lambda u_{n}-\Delta_{p} u_{n}+\gamma\left|D u_{n}\right|^{p}+\gamma \geq 0 \quad \text { in } \Omega^{n}
$$

hence by comparison we have that

$$
\lambda\left\|u_{n}^{-}\right\|_{L^{\infty}\left(\Omega^{n}\right)} \leq \gamma,
$$

i.e. the sequence $\left\{u_{n}\right\}$ is uniformly bounded from below.

We divide the proof into several steps.

Step 1. Let us define $\varphi_{\eta}(s)$ as

$$
\varphi_{\eta}(s)=s e^{\eta s^{2}} \quad \eta>0,
$$

and notice that it satisfies the following property:

$$
\forall a \in \mathbb{R}^{+} \exists \eta, b \in \mathbb{R}^{+} \text {such that : } \varphi_{\eta}^{\prime}(s)-a\left|\varphi_{\eta}(s)\right| \geq b .
$$

Choose $\varphi_{\eta}\left(k-T_{k}\left(u_{n}\right)\right)$, for any $k \geq \frac{\eta}{\lambda}$, as test function in 4.1., so that

$$
\int_{\Omega^{n}}\left|D T_{k}\left(u_{n}\right)\right|^{p} \varphi_{\eta}^{\prime}\left(k-T_{k}\left(u_{n}\right)\right)=\int_{\Omega^{n}} H\left(x, D u_{n}\right) \varphi_{\eta}\left(k-T_{k}\left(u_{n}\right)\right)+\lambda \int_{\Omega^{n}} u_{n} \varphi_{\eta}\left(k-T_{k}\left(u_{n}\right)\right)
$$


which implies, using 2.23,

$$
\int_{\Omega^{n}}\left|D T_{k}\left(u_{n}\right)\right|^{p} \varphi_{\eta}^{\prime}\left(k-T_{k}\left(u_{n}\right)\right) \leq \int_{\Omega^{n}} \gamma\left|D T_{k}\left(u_{n}\right)\right|^{p} \varphi_{\eta}\left(k-T_{k}\left(u_{n}\right)\right)+(\lambda k+\gamma) \varphi_{\eta}(2 k)|\Omega| .
$$

Thanks to the property of $\varphi_{\eta}$, choosing $\eta$ large enough (independent from $k$ ) we deduce that $k-T_{k}\left(u_{n}\right)$ is bounded in $W_{0}^{1, p}\left(\Omega^{n}\right)$.

Step 2. Fix any $\varepsilon<\beta$, and set $\delta=\delta_{\varepsilon}$ and $\Omega_{\delta}$, a neighborhood of $\partial \Omega$, be given by $(2.22)$, so that

$$
H(x, \xi) \geq(\beta-\varepsilon)|\xi|^{q}-\varepsilon d^{-\frac{q}{q-(p-1)}(x)} \quad \text { in } \Omega_{\delta} .
$$

Now we construct a suitable supersolution $\bar{u}$, such that

$$
u_{n} \leq \bar{u} \quad \text { in } \quad \Omega_{\delta} \quad \forall n \geq n_{0} .
$$

To this purpose, we fix any $\eta>0$ small enough and we consider the strip

$$
A_{\eta}^{\delta-\eta}:=\Omega_{\delta-\eta} \backslash \Omega_{\eta}=\{x \in \Omega: \eta<d(x)<\delta-\eta\} ;
$$

if $p-1<q<p$ we consider

$$
\bar{u}=C_{1} d_{\eta}^{-\alpha}+M
$$

where $d_{\eta}$ is the distance to $\partial A_{\eta}^{\delta-\eta}, C_{1}$ is a positive large number, $\alpha=\frac{p-q}{q-(p-1)}$ and $M>0$.

For a suitable choice of the constants $C_{1}$ and $M$, independent from $\eta$ and $n, \bar{u}$ satisfies

$$
\lambda \bar{u}-\Delta_{p} \bar{u}+(\beta-\varepsilon)|D \bar{u}|^{q} \geq \varepsilon d^{-\frac{q}{q-(p-1)}} \quad \text { in } A_{\eta}^{\delta-\eta} .
$$

Since $\bar{u}$ lies above $u_{n}$ at $\partial A_{\eta}^{\delta-\eta}$ for any $\eta>0$, we deduce, using a comparison principle (see Theorem 1.2 in [25]) for $W^{1, p} \cap L^{\infty}$ solutions, that $u_{n} \leq \bar{u}$ in $A_{\eta}^{\delta-\eta}$, for any $\eta>0$ and $n \geq n_{0}$. If $q=p$, we proceed as before using $\bar{u}=-C_{1} \log d_{\eta}+M$. Letting $\eta \rightarrow 0$, we deduce (4.3).

Step 3. Local bound in $L^{\infty} \cap W^{1, p}$

As a consequence of the estimate in $(4.3)$, we deduce that there exists a strip

$$
A_{\delta_{0}}^{\delta_{1}}=\left\{x \in \Omega: \delta_{0}<d(x)<\delta_{1}\right\} \quad \text { for some } 0<\delta_{0}<\delta_{1}<\delta
$$

and a constant $c_{\delta_{0}, \delta_{1}}$ such that $u_{n}(x) \leq c_{\delta_{0}, \delta_{1}}$ in $A_{\delta_{0}}^{\delta_{1}}$. Thus we can consider the following test function in the weak formulation of 4.1 :

$$
\psi= \begin{cases}e^{\mu\left(u_{n}-k\right)^{+}}-1 & \text { in } \Omega \backslash \Omega_{\delta_{0}} \\ 0 & \text { in } \Omega_{\delta_{0}}\end{cases}
$$

for any $\mu>0$ and $k \geq \max \left\{c_{\delta_{0}, \delta_{1}}, \frac{\gamma_{1}}{\lambda}\right\}$.

Observe that $\psi$ has compact support in $\Omega$. Hence, using the lower bound in $(2.23$ and the classical Stampacchia's method (see [36]) we deduce that $u_{n}$ is uniformly bounded in $\Omega \backslash \Omega_{\delta_{0}}$. Since $u_{n} \leq \bar{u}$ in $\Omega_{\delta_{0}}$, we conclude that $u_{n}$ is bounded on any compact subset of $\Omega$.

Using now the natural growth condition 2.23 , it easily follows from the local uniform bound in $L^{\infty}$ that

$$
\forall \omega \subset \subset \Omega, \quad \exists c=c(\omega, \Omega)>0: \quad\left\|u_{n}\right\|_{W^{1, p}(\omega)} \leq c .
$$

Step 4. Compactness.

By nowadays classical arguments (see e.g. [13]), it follows that $u_{n}$ is locally compact in $W^{1, p}(\Omega)$ and, subsequently, that $H\left(x, D u_{n}\right)$ is locally compact in $L^{1}(\Omega)$. As a consequence of the compactness result, there exists a function $u \in W_{\mathrm{loc}}^{1, p}(\Omega) \cap L_{\mathrm{loc}}^{\infty}(\Omega)$ such that $u_{n}$ converges, up to subsequences, to $u$ which satisfies

$$
\lambda u-\Delta_{p} u+H(x, D u)=0 \quad \text { in } \Omega .
$$


Finally, thanks to the boundedness of $k-T_{k}\left(u_{n}\right)$ in $W_{0}^{1, p}\left(\Omega_{n}\right)$, we also deduce that $k-T_{k}(u)$ belongs to $W_{0}^{1, p}(\Omega)$, so that $u$ is a solution to $\left(P_{\lambda}\right)$.

4.2. Uniqueness. The uniqueness of the large solution is obtained as the consequence of two facts: the first one is the precise behavior at the boundary of any large solution to $\left[E_{\lambda}\right]$, the second one is a comparison principle for bounded solution at the interior of $\Omega$.

To this purpose, we need to use comparison principles for $p$-Laplace equations with first order terms. For an overview of comparison results in this framework, we refer the reader to [8, 25] and 31 (see also the references cited therein). Here, we only recall those general comparison principles that we are going to apply systematically in this and in the next Section.

We consider the equation

$$
\lambda u-\Delta_{p} u+H(x, D u)=0 \quad \text { in } \mathcal{O},
$$

for $\lambda \geq 0, \mathcal{O}$ a bounded subset of $\mathbb{R}^{N}, N \geq 2$ and $H(x, \xi)$ a suitable Carathéodory function.

Theorem 4.1 (Theorem 1.2 and Theorem 2.3 ii) in 25]). Let $u, v$ be $W^{1, p}(\mathcal{O}) \cap L^{\infty}(\mathcal{O})$ sub and supersolutions to (4.4) respectively, and assume that $H(x, \xi)$ satisfies one of the following set of assumptions for either $\tau=1$ or $\tau=-1$ :

i) $\lambda>0$ and there exists $c>0$ such that

$$
\begin{aligned}
& \tau\left[H_{\xi}(x, \xi) \cdot \xi-(p-1) H(x, \xi)\right] \geq-c\left(1+|\xi|^{p}\right), \\
& \left|H_{\xi}(x, \xi)\right| \leq c\left(1+|\xi|^{p-1}\right),
\end{aligned}
$$

for a.e. $x \in \mathcal{O}$, and for any $\xi \in \mathbb{R}^{N}$;

ii) $\lambda \geq 0$ and there exists $c>0$ such that

$$
\begin{aligned}
& \tau\left[H_{\xi}(x, \xi) \cdot \xi-(p-1) H(x, \xi)\right] \geq-c|\xi|^{p}+\mu \quad \text { for some } \mu>0, \\
& \left|H_{\xi}(x, \xi)\right| \leq c\left(1+|\xi|^{p-1}\right),
\end{aligned}
$$

for a.e. $x \in \mathcal{O}$, and for any $\xi \in \mathbb{R}^{N}$;

iii) $\lambda=0$, hypothesis 4.5 holds and at least one between the subsolution $u$ or the supersolution $v$ is strict.

iv) $\lambda>0, u, v \in W^{1, \infty}(\mathcal{O})$ and $\xi \mapsto H(x, \xi)$ is locally Lipschitz continuous;

If $u \leq v$ at $\partial \mathcal{O}$, then $u \leq v$ in $\mathcal{O}$.

Let us also recall the following comparison principle for the general equation

$$
-\Delta_{p} u+\mathcal{H}(x, u, D u)=0 \quad \text { in } \mathcal{O},
$$

where $\mathcal{H}(x, s, \xi)$ is a Carathéodory function.

Theorem 4.2 (Theorem 1.3 in 25). Let $u, v$ be $W^{1, p}(\mathcal{O}) \cap L^{\infty}(\mathcal{O})$ sub and supersolutions to (4.7) respectively, and assume that $\mathcal{H}(x, s, \xi)$ satisfies the following condition:

for every $\varepsilon>0$ sufficiently small there exists $c_{\varepsilon}$ such that

$$
\mathcal{H}(x, s, \xi)-(1-\varepsilon)^{p-1} \mathcal{H}\left(x, \frac{t+\varepsilon K}{1-\varepsilon}, \frac{\eta}{1-\varepsilon}\right) \leq c_{\varepsilon}|\xi-\eta|^{p-1}(1+|\xi-\eta|) \quad \forall s \leq t,
$$

for some $K>0$, for a.e. $x \in \mathcal{O}$, for any $\xi, \eta \in \mathbb{R}^{N}$ and for $s, \frac{t+\varepsilon K}{1-\varepsilon} \in[-M, M], M=$ $\max \left(\|u\|_{L^{\infty}(\mathcal{O})},\|v\|_{L^{\infty}(\mathcal{O})}\right)$.

If $u \leq v$ at $\partial \mathcal{O}$, then $u \leq v$ in $\mathcal{O}$.

A crucial step towards the uniqueness result is the proof of some qualitative properties of large solutions, specifically the asymptotic profile of $u$, as well as of its normal derivative, at the boundary. We summarize them in the following Proposition. 
Proposition 4.3. Assume that hypotheses 2.22) 2.24 hold true, let $\lambda>0$ and let $u$ be any solution to $\left(P_{\lambda}\right)$ according to Definition 2.10. Then u satisfies:

i) $u \in C^{0, \alpha}(\Omega)$ for some $\alpha \in(0,1)$;

ii) $u(x)$ tends to $+\infty$ pointwisely at $\partial \Omega$;

iii) if $p-1<q<p$,

where

$$
\alpha=\frac{p-q}{q-(p-1)} \quad \text { and } \quad C_{q}=\left(\frac{1}{\beta} \frac{p-1}{q-(p-1)}\right)^{\frac{1}{q-(p-1)}} \frac{q-(p-1)}{p-q}=\frac{1}{\alpha}\left[\frac{(p-1)(\alpha+1)}{\beta}\right]^{\alpha+1}
$$

while if $q=p$,

$$
\lim _{d(x) \rightarrow 0} \frac{u(x)}{-\log (d(x))}=\frac{p-1}{\beta},
$$

iv) for any $p-1<q \leq p$, we have that

$$
\lim _{d(x) \rightarrow 0} d^{\alpha+1}(x) D u \cdot \nu=\alpha C_{q} \quad \text { and } \quad D u \cdot \tau=o(D u \cdot \nu) \quad \text { as } d(x) \rightarrow 0,
$$

(with $\alpha=0$ and $C_{p} \alpha=\frac{p-1}{\beta}$ if $p=q$ ).

Remark 4.4. Here the condition $\lambda>0$ is only used in the proof of (iii) above. However, in the study of the ergodic problem (see Section 5) a similar estimate will be proved for the case $\lambda=0$ as well. We stress that, once (iii) is proved, then (iv) follows without further condition. Therefore, in the end the conclusions of the above result do not actually depend on $\lambda$.

\section{Proof of Proposition 4.3 .}

i) The interior $C^{0, \alpha}$ regularity is a consequence of classical results (see [16]).

ii) Suppose that $u$ is a solution to $E_{\lambda}$ in the sense of Definition 2.10 Let us define, for any $k>0$ and $0<\delta<k$, the following $W^{1, \infty}(\mathbb{R})$ function

$$
\eta_{\delta, k}(s)=\left\{\begin{array}{lll}
1 & \text { if } \quad s \leq k-\delta \\
\frac{k-s}{\delta} & \text { if } \quad k-\delta \leq s \leq k \\
0 & \text { if } \quad s \geq k
\end{array}\right.
$$

and let us choose $\psi=\Psi \eta_{\delta, k}(u)$, where $\Psi \in W_{\text {loc }}^{1, p}(\Omega) \cap L_{\text {loc }}^{\infty}(\Omega), \Psi \geq 0$. Developing the computations we deduce, using that $\eta_{\delta, k}^{\prime}(s) \leq 0$ :

$$
\int_{\Omega} \lambda u \Psi \eta_{\delta, k}(u)+\int_{\Omega} \eta_{\delta, k}(u)|D u|^{p-2} D u D \Psi+\int_{\Omega} H(x, D u) \Psi \eta_{\delta, k}(u) \geq 0 .
$$

We can now pass to the limit as $\delta$ goes to 0 in the above identity and we have that:

$$
\int_{\Omega} \lambda u \Psi \chi_{\{u \leq k\}}+\int_{\Omega}\left|D T_{k}(u)\right|^{p-2} D T_{k}(u) \cdot D \Psi+\int_{\Omega} H(x, D u) \Psi \chi_{\{u \leq k\}} \geq 0 .
$$

Using (2.23), we deduce in particular that $T_{k}(u)$, for $k$ large enough, is a super solution to the equation

$$
\lambda \varphi-\Delta_{p} \varphi+\gamma|D \varphi|^{p}=-\gamma
$$

and, by definition, $T_{k}(u)-k \in W_{0}^{1, p}(\Omega) \cap L^{\infty}(\Omega)$. 
We want to prove that this is sufficient to assure that $u$ blows up at the boundary point wise. Let us consider the following $C^{2}(\bar{\Omega})$ function:

$$
v(x)=-\frac{p-1}{\gamma} \log \left(d(x)+e^{-\frac{\gamma}{p-1} k}\right)-M, \quad M>0 .
$$

Observe that it turns out to be a subsolution to 4.13 and that $v \leq k$ in $\Omega$. Thus, using the comparison principle due to Theorem 4.1. we deduce that $v \leq T_{k}(u)$ in $\Omega$, for any $k$ large enough. In particular, this means that

$$
-\frac{p-1}{\gamma} \log \left(d(x)+e^{-\frac{\gamma}{p-1} k}\right)-M \leq T_{k}(u) \leq u \quad \text { in } \Omega .
$$

Consequently, letting $k \rightarrow \infty$, we deduce that $u$ satisfies

$$
-\frac{p-1}{\gamma} \log (d(x))-M \leq u \quad \text { in } \Omega,
$$

hence $u$ blows up at the boundary pointwisely.

iii) By assumption 2.22, given $\varepsilon>0$ we have that $u$ is a super solution to

$$
\lambda \varphi-\Delta_{p} \varphi+(\beta+\varepsilon)|D \varphi|^{q}=-\varepsilon d^{-\frac{q}{q-(p-1)}}(x)
$$

and a sub solution to

$$
\lambda \varphi-\Delta_{p} \varphi+(\beta-\varepsilon)|D \varphi|^{q}=\varepsilon d^{-\frac{q}{q-(p-1)}}(x)
$$

in a suitable neighborhood of the boundary $\Omega_{\delta_{\varepsilon}}$.

Observe that the one dimensional function $v(t)=C_{q} t^{-\alpha}$, where $C_{q}$ and $\alpha$ have been defined in 4.10, satisfies, if $p-1<q<p$,

$$
\left(\left|v^{\prime}\right|^{p-2} v^{\prime}\right)^{\prime}=\beta\left|v^{\prime}\right|^{q} \quad \text { for } \quad t>0, \quad \text { and } \quad \lim _{t \rightarrow 0^{+}} v(t)=+\infty,
$$

while $v(t)=-(p-1) / \beta \log (t)$ solves

$$
\left(\left|v^{\prime}\right|^{p-2} v^{\prime}\right)^{\prime}=\beta\left|v^{\prime}\right|^{p} \quad \text { for } \quad t>0, \quad \text { and } \quad \lim _{t \rightarrow 0^{+}} v(t)=+\infty .
$$

Hence we construct suitable super and subsolutions to (4.14) and (4.15), respectively.

We start by proving the result in the case $p-1<q<p$. Here we can choose $\eta_{\varepsilon}$ such that $\eta_{\varepsilon} \rightarrow 0$ as $\varepsilon \rightarrow 0$ and the function

$$
\underline{u}=\left(1-\eta_{\varepsilon}\right) C_{q}(d(x)+\delta)^{-\alpha}-\frac{M_{\varepsilon}}{\lambda}
$$

is a subsolution to (4.14) in $\Omega_{\delta_{\varepsilon}}$, for any $\delta \in\left(0, \delta_{\varepsilon}\right)$, with $\delta_{\varepsilon}$ sufficiently small. Notice that $\underline{u} \in C^{2}\left(\overline{\Omega_{\delta_{\varepsilon}}}\right)$ and that $\underline{u}<+\infty$ at $\partial \Omega$ so that $\operatorname{supp}(\underline{u}-u)^{+}$is a compact set $\omega \subset \subset \Omega_{\delta_{\varepsilon}}$ provided $M_{\varepsilon}$ is chosen sufficiently large. Applying Theorem 4.1 in a sequence of smooth subsets $\omega_{j} \nearrow \Omega_{\delta_{\varepsilon}}$, we deduce that $\underline{u} \leq u$ in $\Omega_{\delta_{\varepsilon}}$, and consequently

$$
\frac{d^{\alpha}(x) u(x)}{C_{q}} \geq\left(1-\eta_{\varepsilon}\right) \frac{d^{\alpha}(x)}{(d(x)+\delta)^{\alpha}}-\frac{d^{\alpha}(x)}{C_{q}} \frac{M_{\varepsilon}}{\lambda} \quad \text { in } \Omega_{\delta_{\varepsilon}} .
$$

Letting $\delta$ vanish we deduce that

$$
\lim _{d(x) \rightarrow 0} \frac{d^{\alpha}(x) u(x)}{C_{q}} \geq 1-\eta_{\varepsilon} .
$$

Arguing as before, we also have that

$$
\bar{u}=\left(1+\eta_{\varepsilon}\right) C_{q}(d(x)-\delta)^{-\alpha}+\frac{M_{\varepsilon}}{\lambda}
$$


turns out to be a supersolution to 4.15 in a strip of the form $A_{\delta}^{\delta_{\varepsilon}}=\left\{x \in \Omega: \delta<\mathrm{d}(x, \partial \Omega)<\delta_{\varepsilon}\right\}$. Thus we have that

$$
\frac{d^{\alpha}(x) u(x)}{C_{q}} \leq\left(1+\eta_{\varepsilon}\right) \frac{d^{\alpha}(x)}{(d(x)-\delta)^{\alpha}}+\frac{d^{\alpha}(x)}{C_{q}} \frac{M_{\varepsilon}}{\lambda} \quad \text { in } A_{\delta}^{\delta_{\varepsilon}},
$$

and letting $\delta$ vanish, we obtain

$$
\lim _{d(x) \rightarrow 0} \frac{d^{\alpha}(x) u(x)}{C_{q}} \leq 1+\eta_{\varepsilon} .
$$

Letting $\varepsilon \rightarrow 0, \eta_{\varepsilon}$ vanishes and we get (4.9).

The case $p=q$ follows just by changing the power $C_{q} d^{-\alpha}$ with $-\frac{p-1}{\beta} \log d(x)$.

iv) Let $x_{0}$ be a point of $\partial \Omega$ and let us consider a point $P_{0} \in \Omega$ close to the boundary such that its projection on $\partial \Omega$ coincides with $x_{0}$. In $B\left(P_{0}, d_{0}\right), d_{0}=\left|x_{0}-P_{0}\right|$, we set a new system of coordinates $\left(\eta_{1}, \ldots, \eta_{N}\right)=\left(\eta_{1}, \eta^{\prime}\right)$ such that the origin $O$ coincides with $x_{0}$ and the $\eta_{1}$-axis coincides with $\operatorname{Dd}\left(x_{0}\right)=-\nu\left(x_{0}\right)$.

We first consider the case $q<p$. Let us set, for any $\delta>0, \varsigma=\frac{\eta}{\delta}$ and let us define $u_{\delta}(\varsigma)=$ $u(\delta \varsigma) \delta^{\alpha}$, where $\alpha$ is defined in 4.10 and $u$ is a solution to $\left(P_{\lambda}\right)$. Hence $u_{\delta}(\varsigma)$ solves

$$
\lambda \delta^{(p-2)(\alpha+1)+2} u_{\delta}-\Delta_{p} u_{\delta}+\delta^{(p-1)(\alpha+1)+1} H\left(\delta \varsigma, \delta^{-(\alpha+1)} D u_{\delta}\right)=0 \quad \text { in } D_{\delta},
$$

where $D_{\delta}=\left\{\left(\varsigma_{1}, \varsigma^{\prime}\right):\left(\varsigma_{1}-d_{0} / \delta\right)^{2}+\left|\varsigma^{\prime}\right|^{2} \leq\left(d_{0} / \delta\right)^{2}\right\}$. Notice that the estimate on the asymptotic behavior of $u$ implies $u_{\delta}(\varsigma) \leq \frac{C}{\varsigma_{1}^{\alpha}}$, so that the function $u_{\delta}(\varsigma)$ is locally bounded; and more precisely, due to 4.9 , we have that, as $\delta$ vanishes, $u(\delta \varsigma) \delta^{\alpha} \rightarrow C_{q} \varsigma_{1}^{-\alpha}$, where $C_{q}$ is defined in (4.10. Assuming that $d_{0}$ is sufficiently small, we deduce from 2.22 that

$$
|H(x, \xi)| \leq c\left(|\xi|^{q}+\frac{1}{d^{\frac{q}{q-(p-1)}(x)}}\right) \quad \forall x \in B\left(P_{0}, d_{0}\right), \quad \forall \varsigma \in \mathbb{R}^{N},
$$

for some constants $c>0$. Therefore, using the exact value of $\alpha$ we conclude that

$$
\delta^{(p-1)(\alpha+1)+1}\left|H\left(\delta \varsigma, \delta^{-(\alpha+1)} D u_{\delta}\right)\right| \leq c\left|D u_{\delta}\right|^{q}+\omega(\varsigma),
$$

where $\omega(\varsigma)$ is a locally bounded function in $D_{\delta}$, and therefore, from 4.18), we deduce that $u_{\delta}$ satisfies

$$
-\Delta_{p} u_{\delta}=\tilde{H}\left(\varsigma, u_{\delta}, D u_{\delta}\right) \quad \text { in } D_{\delta}
$$

for some $\tilde{H}(\varsigma, s, \xi)$ which has natural growth with respect to $\xi$. Thanks to [16], this implies that $u_{\delta}$ is bounded in $C_{\text {loc }}^{1, \alpha}\left(D_{\delta}\right)$ for some $\alpha>0$, and so it is locally compact in $C^{1}\left(D_{\delta}\right)$. Notice that $D_{\delta} \rightarrow \mathbb{R}_{+}^{N}$ as $\delta$ vanishes, so that $u_{\delta}$ is compact in $C_{\text {loc }}^{1}\left(\mathbb{R}_{+}^{N}\right)$. Consequently, $D u_{\delta}$ converges locally uniformly toward $D C_{q} \varsigma_{1}^{-\alpha}=-\alpha C_{q} \varsigma_{1}^{-\alpha-1} e_{1}$. In particular, we have that

$$
\delta^{\alpha+1} D u(\delta, 0, \ldots, 0)=D u_{\delta}(1,0, \ldots, 0) \rightarrow-\alpha C_{q} e_{1}
$$

which implies 4.12, by arbitrariness of $x_{0}$.

Consider now $q=p$. We define $u_{\delta}(\varsigma)=u(\delta \varsigma)+\frac{p-1}{\beta} \log (\delta)$ and thus $u_{\delta}$ solves

$$
\lambda \delta^{p}\left[u_{\delta}-(p-1) \log (\delta)\right]-\Delta_{p} u_{\delta}+\delta^{p} H\left(\delta \varsigma, \delta^{-1} D u_{\delta}\right)=0 \quad \text { in } D_{\delta} .
$$

According to 2.22 2.23 , given a ball $B_{R}$, for every $\varepsilon>0$ there exists $\delta_{\varepsilon}$ such that for any $\varsigma \in D_{\delta} \cap B_{R}$ we have

$$
(\beta-\varepsilon)\left|D u_{\delta}\right|^{p}+\varepsilon \omega(\varsigma) \leq \delta^{p} H\left(\delta \varsigma, \delta^{-1} D u_{\delta}\right) \leq(\beta+\varepsilon)\left|D u_{\delta}\right|^{p}+\delta^{p} c_{\gamma},
$$


for all $\delta<\delta_{\varepsilon}$, where $\omega$ is some bounded function in $D_{\delta} \cap B_{R}$. Consequently, once again thanks to [16], $u_{\delta}(\varsigma)$ converges in $C_{\mathrm{loc}}^{1}\left(D_{\delta}\right)$ and, in addition, $u$ is a solution to

$$
\left\{\begin{array}{l}
-\Delta_{p} u+\beta|D u|^{p}=0 \quad \text { in } \mathbb{R}_{+}^{N}, \\
u_{\left.\right|_{\varsigma_{1}=0}}=+\infty
\end{array}\right.
$$

where last condition is a consequence of the lower bound given by 4.11). Now, the above equation implies that $e^{-\frac{\beta}{p-1} u}$ is a $p$-harmonic function that vanishes at $\varsigma_{1}=0$. We deduce (see [19]) that $e^{-\frac{\beta}{p-1} u}$ is flat, and consequently that $u$ equals $-\frac{p-1}{\beta} \log \left(\varsigma_{1}\right)$ up to a constant. Hence 4.12 holds true.

\section{Proof of Theorem 2.11; Uniqueness.}

Assume that $\left(P_{\lambda}\right)$ possesses two large solutions $u$ and $v$. For any $\varepsilon \in(0,1)$ sufficiently small and for a suitable $M>0$ to be fixed later, we first define $u_{\varepsilon}=(1-\varepsilon) u-\varepsilon M$, that satisfies

$$
\begin{gathered}
\lambda u_{\varepsilon}-\Delta_{p} u_{\varepsilon}+H\left(x, D u_{\varepsilon}\right)=\lambda(1-\varepsilon)\left(1-(1-\varepsilon)^{p-2}\right) u-\lambda \varepsilon M \\
+H(x, D u(1-\varepsilon))-(1-\varepsilon)^{p-1} H(x, D u) .
\end{gathered}
$$

Using (2.26), we deduce

$$
\lambda u_{\varepsilon}-\Delta_{p} u_{\varepsilon}+H\left(x, D u_{\varepsilon}\right) \leq-\varepsilon\left[\rho(x)|D u|^{q}-\sigma(x)-\lambda c_{p} u+\lambda M\right] .
$$

Using estimates iii) and iv) in Proposition 4.3 and since $\rho(x)$ is coercive near the boundary, we know that there exist $c_{1}$ and $c_{2}>0$ such that

$$
\left[\rho(x)|D u|^{q}-\sigma(x)-\lambda c_{p} u+\lambda M\right] \geq\left[c_{1} d^{-q(\alpha+1)}(x)-\sigma(x)-c_{2} d^{-\alpha}(x)+M\right]
$$

in a suitable neighborhood $\Omega_{\delta}$ of the boundary. Since $q(\alpha+1)=\frac{q}{q-(p-1)}$ and

$d^{\frac{q}{q-(p-1)}}(x) \sigma(x) \rightarrow 0$ as $d(x) \rightarrow 0$, we conclude that the right hand side of 4.20$)$ is negative in $\Omega_{\delta}$ for some $\delta>0$. Then, choosing $M$ large enough, it will be negative in $\Omega \backslash \Omega_{\delta}$ as well, because $u$ and $\sigma$ are locally bounded. Therefore, we conclude from 4.20 that $u_{\varepsilon}$ is a subsolution in $\Omega$.

We can now compare $u_{\varepsilon}$ and $v$ on any compact subset $\omega$ of $\Omega$, where the two functions are, respectively, a sub and a super solution belonging to $W^{1, p}(\omega) \cap L^{\infty}(\omega)$. In particular, if 2.25 is satisfied, then condition 4.5 holds for $\tau=1$ (using also (2.23) ) and we can apply Theorem 4.1 . If instead 2.27) holds, then we can apply directly Theorem 4.2 with $\mathcal{H}(x, s, \xi)=\lambda s+H(x, \xi)$.

Finally, observe that $u_{\varepsilon}-v \rightarrow-\infty$ as $x \rightarrow \partial \Omega$ due to the asymptotic behavior (4.9) or (4.11). Thus, we can apply the comparison principle on a sequence of smooth subsets $\omega_{j} \nearrow \Omega$, obtaining that $u_{\varepsilon} \leq v$ in $\Omega$. Letting $\varepsilon \rightarrow 0$ we conclude that $u \leq v$, and reversing the roles of $u$ and $v$ the uniqueness is proved.

Remark 4.5. Let us remark that the approach used here to the existence of large solutions takes profit of the natural growth of the Hamiltonian and this allows us to prove uniqueness in a weak formulation.

In more generality, strengthening the hypothesis on $H$ and using the local gradient estimates of Theorem 2.5, one might prove existence and uniqueness of large solutions (in a strong formulation, i.e. solutions are locally Lipschitz in $\Omega)$ whenever $H(x, D u)$ behaves as $x \rightarrow \partial \Omega$ as $h\left(|D u|^{p-1}\right)$, with $\int^{\infty} \frac{1}{h(s)} d s<\infty$ and $\int^{\infty} \frac{s^{\frac{1}{p-1}}}{h(s)} d s=\infty$. This latter condition allows for the construction of suitable one-dimensional comparison functions blowing up at the boundary, as in Proposition 4.3 (iii). Jointly with a local gradient bound, this would easily yield the existence of solutions. As for uniqueness, the main point is to show that any solution has the same first 
order asymptotics as $d(x)$ tends to zero. Once this is achieved, one can use Theorem 4.1 (iv) for the comparison principle inside $\Omega$.

\section{Proof of the ReSults on the ERgodic Problem}

We start this section by proving the existence result for the ergodic problem.

\section{Proof of Theorem 2.15.}

\section{Step 1. Estimates on $u_{\lambda}$.}

We start by observing that, by (4.16) and (4.17), for $\varepsilon$ small enough, there exists $\eta_{\varepsilon} \rightarrow 0$ and $M_{\varepsilon}>0$ such that

$$
\lambda\left(1-\eta_{\varepsilon}\right) C_{q} d^{-\alpha}(x)-M_{\varepsilon} \leq \lambda u(x) \leq \lambda\left(1+\eta_{\varepsilon}\right) C_{q} d^{-\alpha}(x)+M_{\varepsilon} \quad \text { in } \Omega,
$$

if $q \in(p-1, p)$ and

$$
-\left(1-\eta_{\varepsilon}\right) \lambda(p-1) \log d(x)-M_{\varepsilon} \leq \lambda u(x) \leq-\left(1+\eta_{\varepsilon}\right) \lambda(p-1) \log d(x)+M_{\varepsilon} \quad \text { in } \Omega
$$

if $q=p$; consequently, in both cases,

$$
\forall \omega \subset \subset \quad \exists c_{\omega}>0: \quad\left\|\lambda u_{\lambda}(x)\right\|_{L^{\infty}(\omega)} \leq c_{\omega} .
$$

On the other hand, as we stressed in Remark 2.6, the local estimate on $\left|D u_{\lambda}\right|$ only depends on $\left\|\lambda u_{\lambda}(x)\right\|_{L^{\infty}(\omega)}$, so that by Theorem 2.5 we deduce that $\left\|D u_{\lambda}(x)\right\|_{L^{\infty}(\omega)} \leq c_{\omega}$, too.

This means that $\lambda u_{\lambda}(x)$ is bounded in $W^{1, \infty}(\omega), \forall \omega \subset \subset \Omega$ and

$$
\left\|D \lambda u_{\lambda}(x)\right\|_{L^{\infty}(\omega)} \leq \lambda c_{\omega} .
$$

Thus $\left|D \lambda u_{\lambda}(x)\right| \rightarrow 0$ uniformly in any $\omega \subset \subset \Omega$. Since $\Omega$ is connected, there exists a constant $c_{0}$ such that

$$
\lim _{\lambda \rightarrow 0} \lambda u_{\lambda}(x)=c_{0} \quad \text { locally uniformly in } \Omega .
$$

Step 2. Estimates on $v_{\lambda}:=u_{\lambda}-u_{\lambda}\left(x_{0}\right)$.

First of all $v_{\lambda}\left(x_{0}\right)=0, \forall \lambda>0$. Moreover $D v_{\lambda}(x)=D u_{\lambda}(x)$ and consequently, due to Step 1, for any $\omega \subset \subset \Omega, \omega$ connected, there exists a constant (we still denote it by $c_{\omega}$ ) such that

$$
\left\|D v_{\lambda}\right\|_{L^{\infty}(\omega)}+\left\|v_{\lambda}\right\|_{L^{\infty}(\omega)} \leq c_{\omega}
$$

This means that there exists a continuous function $v(x)$ such that, up to extract a subsequence (not relabeled),

$$
v_{\lambda}(x) \rightarrow v(x) \quad \text { locally uniformly in } \Omega
$$

Step 3. $v$ solves 2.31.

By standard compactness arguments, it is easy to see that $v$ is a weak solution of the equation inside $\Omega$.

Let us prove that it satisfies the explosive boundary condition. Fix $0<\delta_{0}<\underline{\delta}$ (recall that $\underline{\delta}$ has been defined in (1.7) ) small enough: using the uniform estimate (5.3), we deduce that there exists $c_{\delta_{0}}>0$ such that $\left|v_{\lambda}(x)\right| \leq c_{\delta_{0}}$ in $\Omega \backslash \Omega_{\frac{\delta_{0}}{2}}$.

We consider, for any $\sigma \in(0,1)$ small enough and $\delta$ small enough, too, the function $z_{\delta}(x)=$ $-\frac{p-1}{\gamma} \sigma \log \left(\frac{d+\delta}{\delta_{0}+\delta}\right)-c_{\delta_{0}}$ in $\Omega_{\delta_{0}}$. We have

$$
\lambda z_{\delta}-\Delta_{p} z_{\delta}+\gamma\left(\left|D z_{\delta}\right|^{p}+1\right) \leq 0 \quad \text { in } \Omega_{\delta_{0}},
$$

provided $\delta_{0}$ is small. On the other hand, by 2.23$)$, and since $\lambda u_{\lambda}\left(x_{0}\right)$ is bounded, we have that $v_{\lambda}$ is a super solution of the same equation (eventually replacing $\gamma$ with a larger value). Hence, by Theorem $4.1 \mathrm{i}), v_{\lambda} \geq z_{\delta}$. Letting $\delta$ vanishing and $\lambda \rightarrow 0$, we deduce that $v$ blows up at $\partial \Omega$. 
Step 4. We prove that any solution $v$ to 2.31 has the same behavior at the boundary.

In order to do it, we proceed as in the proof of Proposition 4.3. For $q<p$ and $\varepsilon$ arbitrarily small, let $\delta_{\varepsilon} \in(0, \bar{\delta})$ be given by 2.22); then $v$ satisfies

$$
c_{0}-\Delta_{p} v+(\beta-\varepsilon)|D v|^{q} \leq \varepsilon d^{-\frac{q}{q-(p-1)}}(x)
$$

in $\Omega_{\delta_{\varepsilon}}$. On the other hand, for any $\delta \in\left(0, \delta_{\varepsilon}\right)$ the function

$$
z_{\delta}(x)=\frac{C_{q}+\eta_{\varepsilon}}{(d-\delta)^{\alpha}}+M_{\varepsilon} \quad \text { where } \quad M_{\varepsilon}=\max _{d=\delta_{\varepsilon}} v(x)
$$

turns out to be a strict supersolution of the same equation in the strip $\Omega_{\delta_{\varepsilon}} \backslash \Omega_{\delta}$, for some convenient $\eta_{\varepsilon}$ such that $\eta_{\varepsilon} \rightarrow 0$ as $\varepsilon \rightarrow 0$. Applying Theorem 4.1.(iii), we deduce that $v \leq z_{\delta}$, and letting $\delta$ vanish, we deduce that

$$
\limsup _{d(x) \rightarrow 0} d^{\alpha}(x) v(x) \leq C_{q}+\eta_{\varepsilon} .
$$

In a similar way we also prove that

$$
\liminf _{d(x) \rightarrow 0} d^{\alpha}(x) v(x) \geq C_{q}-\eta_{\varepsilon}
$$

so that, letting $\varepsilon \rightarrow 0$, we conclude that any solution to 2.31 behaves as $C_{q} d^{-\alpha}$ at $\partial \Omega$.

The case $q=p$ can be treated, as usual, just changing the powers of the distance with the log.

Step 5. $c_{0}$ is unique.

Assume by contradiction that there exists another $c_{1}$ for which 2.31) admits a solution $v_{1}(x)$. Suppose that $c_{0}>c_{1}$ and let us define $v_{\varepsilon}=(1-\varepsilon) v$. Thus, for any $\lambda>0$, using (2.26) we obtain, as in Theorem 2.11, that

$$
\lambda v_{\varepsilon}-\Delta_{p} v_{\varepsilon}+H\left(x, D v_{\varepsilon}\right) \leq-(1-\varepsilon)^{p-1} c_{0}+\lambda(1-\varepsilon) v-\varepsilon\left[\rho(x)|D v|^{q}-\sigma(x)\right],
$$

and so

$$
\begin{gathered}
\lambda v_{\varepsilon}-\Delta_{p} v_{\varepsilon}+H\left(x, D v_{\varepsilon}\right) \leq \lambda v_{1}-c_{1} \\
+c_{1}-c_{0}+\lambda\left[(1-\varepsilon) v-v_{1}\right]-\varepsilon\left[\rho(x)|D v|^{q}-\sigma(x)-c\right] .
\end{gathered}
$$

Thanks to the asymptotic estimates on $v$ and $v_{1}$ proved in the previous step, and consequently on the blow-up of $D v$ (see Remark 4.4), there exists $C>0$ such that, for any $\varepsilon>0$,

$$
\left[(1-\varepsilon) v-v_{1}\right]-\left(\rho(x)|D v|^{q}-\sigma(x)-c\right) \leq C \quad \text { in } \Omega,
$$

since $\rho(x)$ is coercive near the boundary. Therefore, choosing $\lambda=\varepsilon$, we have

$$
\begin{aligned}
\varepsilon v_{\varepsilon}-\Delta_{p} v_{\varepsilon}+H\left(x, D v_{\varepsilon}\right) & \leq \varepsilon v_{1}-c_{1}+c_{1}-c_{0}+\varepsilon C \\
& \leq \varepsilon v_{1}-c_{1}
\end{aligned}
$$

provided $\varepsilon<\frac{c_{0}-c_{1}}{C}$. Therefore $v_{\varepsilon}$ and $v_{1}$ are, respectively, a subsolution and a solution to the same equation, and since $v_{\varepsilon}-v_{1} \rightarrow-\infty$ as $x \rightarrow \partial \Omega$, applying Theorem 4.1 i) we deduce that $(1-\varepsilon) v \leq v_{1}$. Letting $\varepsilon$ vanish, we deduce that $v \leq v_{1}$, and this for any couple of solutions $v, v_{1}$ corresponding to $c_{0}$ and $c_{1}$. Hence we get a contradiction since both $v$ and $v_{1}$ are invariant with respect to addition of constants.

The second result that we prove is a characterization of the constant $c_{0}$ in terms of sub solutions to $E_{\lambda}$.

\section{Proof of Theorem 2.16 .}

We closely follow the proof given in $[32]$ for the case $p=2$, and we prove that the last sentence of the statement is true. To this purpose, assume first that (2.34) admits a solution $v$, then we claim that $c_{0} \geq 0$. Indeed, if $c_{0}<0$, then the solution of (2.31) would be a strict super solution 
to 2.34 and since it blows-up at the boundary, applying Theorem 4.1 iii) in any (sufficiently large) compact subset of $\Omega$, we would deduce that $v \geq u$ in $\Omega$. But this is impossible since $v$ is invariant by addition of a constant. Therefore, problem 2.34 cannot have solutions if $c_{0}<0$.

Assume now that rather $c_{0}>0$ and consider the solutions of the Dirichlet problem

$$
\begin{cases}\lambda u-\Delta_{p} u+H(x, D u)=0 & \text { in } \Omega, \\ u=0 & \text { on } \partial \Omega .\end{cases}
$$

For any $\lambda>0$, there exists a unique solution $u_{\lambda}$ to (5.4) and, by maximum principle,

$\lambda\left\|u_{\lambda}\right\|_{L^{\infty}(\Omega)} \leq C$. By interior gradient estimates, we have that $D u_{\lambda}$ is locally uniformly bounded; therefore, if we fix any $x_{0} \in \Omega$, we have that $u_{\lambda}(\cdot)-u_{\lambda}\left(x_{0}\right)$ is locally bounded in the Lipschitz norm. By standard compactness, it converges, up to subsequences, to a solution $w$ satisfying

$$
\theta-\Delta_{p} w+H(x, D w)=0 \quad \text { in } \Omega,
$$

where $\theta=\lim _{\lambda \rightarrow 0} \lambda u_{\lambda}\left(x_{0}\right)$.

If the Dirichlet problem (2.34) does not have a solution, it can be proved, following [32, that $u_{\lambda}(x) \rightarrow-\infty$ everywhere in $\Omega$, and in this case the function $w$ satisfies the boundary blow-up condition: $w(x) \rightarrow \infty$ as $x \rightarrow \partial \Omega$. Then $w$ is a large solution and, by uniqueness of the ergodic constant in Theorem 2.15 we have $\theta=c_{0}$. So we deduce that $c_{0}=\lim _{\lambda \rightarrow 0} \lambda u_{\lambda}\left(x_{0}\right) \leq 0$.

To sum up, we showed that if (2.34) does not have solutions, then $c_{0} \leq 0$. In other words, $c_{0}>0$ implies the existence of solutions to (2.34).

Let us now set

$$
E:=\left\{c \in \mathbb{R}: \exists \varphi \in W^{1, p}(\Omega) \cap L^{\infty}(\Omega):-\Delta_{p} \varphi+H(x, D \varphi)+c \leq 0\right\} .
$$

Notice that assumption (2.23) implies that conditions 2.16 - 2.17) hold, and in particular Lipschitz barriers can be constructed at the boundary. Therefore, if $c \in E$, reasoning as in Theorem 2.8 the existence of a sub solution would imply the existence of a $W^{1, \infty}(\Omega)$ solution to

$$
-\Delta_{p} \varphi+H(x, D \varphi)+c=0 \quad \text { in } \Omega .
$$

According to the above conclusions, this implies that $c_{0} \geq c$. So $c_{0} \geq \sup E$. But since the ergodic constant relative to $H+c$ is nothing but $c_{0}-c$, applying what we proved in the first part of the Theorem we have that $c_{0}-c>0$ implies the existence of a solution to (5.5). Hence we conclude that $c_{0}=\sup E$. Moreover, the above argument also shows that one can use indifferently $W^{1, \infty}(\Omega)$ sub solutions rather than $W^{1, p}(\Omega) \cap L^{\infty}(\Omega)$, so we also have

$$
c_{0}=\sup \left\{c \in \mathbb{R}: \exists \varphi \in W^{1, \infty}(\Omega):-\Delta_{p} \varphi+H(x, D \varphi)+c \leq 0\right\} .
$$

Before proving Theorem 2.17, we recall the version of the strong comparison principle for equation $\left(E_{0}\right)$ that we are going to use.

Theorem 5.1 (Strong Comparison Principle, Theorem 1.4 in [25]). Let $u, v \in W_{l o c}^{1, \infty}(\Omega)$. Assume that either $u$ or $v$ is a weak solution to

$$
-\Delta_{p} u+\mathcal{H}(x, D u)=0 \text { in } \mathcal{O},
$$

where $\mathcal{H} \in C^{1}\left(\mathcal{O} \times \mathbb{R}^{N}\right)$ satisfies

$$
\mathcal{H}(x, 0)>0 \quad \text { in } \mathcal{O}, \quad(\text { or } \mathcal{H}(x, 0)<0 \quad \text { in } \mathcal{O}),
$$

and

$$
\left|\mathcal{H}_{\xi}(x, \xi)\right| \leq \gamma|\xi|^{\frac{p-2}{2}} \quad \forall \xi \in \mathbb{R}^{N}:|\xi| \leq 1 \quad \text { for a.e. } x \in \mathcal{O} \quad \gamma>0
$$


Assume that

$$
-\Delta_{p} u+\mathcal{H}(x, D u) \leq-\Delta_{p} v+\mathcal{H}(x, D v), \quad u \leq v \quad \text { in } \mathcal{O},
$$

with $\mathcal{O}$ connected. Then $u \equiv v$ in $\mathcal{O}$, unless $u<v$ in $\mathcal{O}$.

\section{Proof of Theorem 2.17,}

Assume that $v$ and $u$ are two solutions of (2.31), with the same ergodic constant $c_{0}$. For any $\varepsilon>0$ small enough, we define $u_{\varepsilon}=(1-\varepsilon) u$, that actually solves

$$
c_{0}-\Delta_{p} u_{\varepsilon}+H\left(x, D u_{\varepsilon}\right)=F_{\varepsilon} \quad \text { in } \Omega
$$

where

$$
F_{\varepsilon}=\left[1-(1-\varepsilon)^{p-1}\right] c_{0}+H\left(x, D u_{\varepsilon}\right)-(1-\varepsilon)^{p-1} H\left(x, \frac{D u_{\varepsilon}}{1-\varepsilon}\right) .
$$

Observe that since $\frac{u}{v} \rightarrow 1$ as $d(x) \rightarrow 0$ (see Step 4 of Theorem 2.15), we have that $u_{\varepsilon}-v \rightarrow-\infty$ as $d(x) \rightarrow 0$. Consequently, using (2.26) and the asymptotic estimates on $D u$ at $\partial \Omega$, we deduce that there exists $\delta>0$ independent from $\varepsilon$ such that

$$
F_{\varepsilon}<0 \quad \text { in } \Omega_{\delta} .
$$

Thus, by Theorem 4.1 iii), we obtain $\max _{\Omega_{\delta}}\left(u_{\varepsilon}-v\right)=\max _{d(x)=\delta}\left(u_{\varepsilon}-v\right)$. Letting $\varepsilon$ vanish, we deduce that

$$
\max _{\Omega_{\delta}}(u-v)=\max _{d(x)=\delta}(u-v) .
$$

On the other hand, in force of assumptions 2.23 and $(2.30)$, on any compact set $\omega$ we have

$$
-c_{\omega} \leq H(x, \xi) \leq \gamma\left(1+|\xi|^{p}\right), \quad\left|H_{\xi}(x, \xi)\right| \leq c_{\omega}\left(1+|\xi|^{p-1}\right)
$$

for some constant $c_{\omega}$. Therefore, since $H(x, 0)+c_{0}>0$, the function $\mathcal{H}(x, \xi)=H(x, \xi)+c_{0}$ satisfies (4.6) with $\tau=-1$, so by using Theorem $4.1 \mathrm{ii}$ ), we have that

$$
u-\left(v+\max _{\partial\left(\Omega \backslash \Omega_{\delta}\right)}(u-v)\right) \leq 0 \quad \text { in } \Omega \backslash \Omega_{\delta}
$$

and consequently

$$
\sup _{\Omega \backslash \Omega_{\delta}}(u-v) \leq \max _{\partial\left(\Omega \backslash \Omega_{\delta}\right)}(u-v) .
$$

It follows from (5.6) and (5.7) that the maximum of $u-v$ is achieved at the set $d(x)=\delta$, and so $u-v$ has an interior maximum. By addition of a constant, we may assume this maximum is zero; therefore, applying the strong comparison principle Theorem 5.1 with $\mathcal{H}(x, \xi)=H(x, \xi)+c_{0}$, we deduce that $u-v$ is a constant.

Finally, we conclude with the proof of Corollary 2.18, which gives more explicit conditions for the uniqueness of large solutions to the ergodic problem.

\section{Proof of Corollary 2.18 .}

By the characterization of $c_{0}$, we immediately deduce that $c_{0}>-\inf H(x, 0)$ and so $c_{0}+$ $H(x, 0)>0$. Now, since

$$
H(x, D \varphi)-H(x, 0) \leq \gamma\left(|D \varphi|+|D \varphi|^{p}\right),
$$

it is well known that there exists a constant $K_{0}>0$ such that the Dirichlet problem

$$
\begin{cases}-\Delta_{p} \varphi+H(x, D \varphi)-H(x, 0)=f(x) & \text { in } \Omega, \\ \varphi=0 & \text { on } \partial \Omega,\end{cases}
$$

admits a solution whenever $\left\|f^{-}\right\|_{L^{\infty}(\Omega)}<K_{0}$. By the way, such a constant can be estimated in terms of a corresponding radial problem through symmetrization method or, otherwise, in 
terms of the eigenvalue of the problem obtained after the Hopf-Cole transformation. Therefore, problem 2.37) admits for sure a solution whenever

$$
\sup _{\Omega}(c+H(x, 0))^{+}<K_{0} \text {. }
$$

If condition 2.38 holds, the above inequality is satisfied for $c=-\inf _{\Omega} H(x, 0)+\varepsilon$, for some $\varepsilon>0$, and we conclude.

\section{Proof of the gradient bounds}

In this Section we develop the well known Bernstein method in order to get local and global bounds for the gradient of solutions to $E_{\lambda}$.

The strategy we use relies on a typical scheme of approximation. Let us recall that the $p$-laplacian operator can be explicitly written, for any $C^{2}$ function $u$, as

$$
\Delta_{p} u=|D u|^{p-2}\left[\Delta u+(p-2) \frac{D^{2} u D u D u}{|D u|^{2}}\right] .
$$

So, for every $v \in C^{1}$, every symmetric $N \times N$ matrix $M$, and every $r \in \mathbb{R}$, we define the functions

$$
\mathcal{A}_{\varepsilon, v}(M)=\operatorname{tr}(M)+(p-2) \frac{M D v D v}{\varepsilon+|D v|^{2}}
$$

and

$$
\alpha_{\varepsilon}(r)=(r+\varepsilon)^{\frac{p-2}{2}}
$$

and, at least for every $z \in C^{2}$, we can approximate the $p$-laplacian of $z$ as

$$
\lim _{\varepsilon \rightarrow 0} \alpha_{\varepsilon}\left(|D z|^{2}\right) \mathcal{A}_{\varepsilon, z}\left(D^{2} z\right)=\alpha_{0}\left(|D z|^{2}\right) \mathcal{A}_{0, z}\left(D^{2} z\right)=\Delta_{p} z
$$

Notice that $\alpha_{\varepsilon}\left(|D u|^{2}\right) \mathcal{A}_{\varepsilon, u}\left(D^{2} u\right)$ defines a quasilinear operator which is smooth and uniformly elliptic: indeed we have

$$
\alpha_{\varepsilon}\left(|D u|^{2}\right) \mathcal{A}_{\varepsilon, u}\left(D^{2} u\right)=\left(|D u|^{2}+\varepsilon\right)^{\frac{p-2}{2}}\left[\delta_{i j}+(p-2) \frac{u_{i} u_{j}}{\varepsilon+|D u|^{2}}\right] u_{i j} \quad i, j=1, \ldots, N
$$

( $\delta_{i j}=1$ if $i=j$ and 0 otherwise) and consequently for any $u \in W^{1, \infty}(\Omega)$ and any $\xi \in \mathbb{R}^{N}$

$$
\varepsilon^{\frac{p-2}{2}}|\xi|^{2} \leq\left[\delta_{i j}+(p-2) \frac{u_{i} u_{j}}{\varepsilon+|D u|^{2}}\right]\left(|D u|^{2}+\varepsilon\right)^{\frac{p-2}{2}} \xi_{i} \xi_{j} \leq(p-1)\left(\|D u\|_{L^{\infty}(\Omega)}^{2}+\varepsilon\right)^{\frac{p-2}{2}}|\xi|^{2} .
$$

Now we can consider the following equation that is nothing but a perturbation of $\left(E_{\lambda}\right)$ :

$$
\lambda u_{\varepsilon}-\alpha_{\varepsilon}\left(\left|D u_{\varepsilon}\right|^{2}\right) \mathcal{A}_{\varepsilon, u_{\varepsilon}}\left(D^{2} u_{\varepsilon}\right)+H\left(x, D u_{\varepsilon}\right)=0 \quad \text { in } \Omega .
$$

Our goal is to prove $L^{\infty}$ a priori estimates for the gradient of the solution of such equation. Let us observe that by the maximum principle any solution of $(6.9)$ satisfies

$$
\lambda\left\|u_{\varepsilon}\right\|_{L^{\infty}(\Omega)} \leq\|H(x, 0)\|_{L^{\infty}(\Omega)}+\lambda \max _{\partial \Omega}\left|u_{\varepsilon}\right| .
$$

The main point of Bernstein's method consists in the following equation solved by $\left|D u_{\varepsilon}\right|^{2}$.

Proposition 6.1. Let $u_{\varepsilon}$ be a solution to 6.9. . Then $w_{\varepsilon}=\left|D u_{\varepsilon}\right|^{2}$ satisfies

$$
\begin{gathered}
\alpha_{\varepsilon}\left(w_{\varepsilon}\right) \mathcal{A}_{\varepsilon, u_{\varepsilon}}\left(D^{2} w_{\varepsilon}\right)=\left[H_{\xi}\left(x, D u_{\varepsilon}\right)-2 \alpha_{\varepsilon}^{\prime}\left(w_{\varepsilon}\right) \mathcal{A}_{\varepsilon, u_{\varepsilon}}\left(D^{2} u_{\varepsilon}\right) D u_{\varepsilon}\right] \cdot D w_{\varepsilon} \\
+2 \lambda w_{\varepsilon}+2 \alpha_{\varepsilon}\left(w_{\varepsilon}\right)\left|D^{2} u_{\varepsilon}\right|^{2}+2 D u_{\varepsilon} \cdot H_{x}\left(x, D u_{\varepsilon}\right) \\
+(p-2) \alpha_{\varepsilon}\left(w_{\varepsilon}\right) \frac{\left(D u_{\varepsilon} \cdot D w_{\varepsilon}\right)^{2}}{\left(\varepsilon+w_{\varepsilon}\right)^{2}}-\frac{p-2}{2} \frac{\alpha_{\varepsilon}\left(w_{\varepsilon}\right)}{\varepsilon+w_{\varepsilon}}\left|D w_{\varepsilon}\right|^{2} \quad \text { in } \Omega .
\end{gathered}
$$


Proof. First of all, we observe that $u_{\varepsilon} \in C^{3}(\Omega)$ due to $\sqrt{2.2}$ and the ellipticity of the operator. For $w_{\varepsilon}=\left|D u_{\varepsilon}\right|^{2}$, we compute

$$
D w_{\varepsilon}=2 D^{2} u_{\varepsilon} D u_{\varepsilon} \quad \text { and } \quad \Delta w_{\varepsilon}=2\left|D^{2} u_{\varepsilon}\right|^{2}+2 D u_{\varepsilon} \cdot D \Delta u_{\varepsilon},
$$

so that

$$
\begin{aligned}
& \alpha_{\varepsilon}\left(\left|D u_{\varepsilon}\right|^{2}\right) \Delta w_{\varepsilon}=\alpha_{\varepsilon}\left(w_{\varepsilon}\right) \Delta w_{\varepsilon}=2 \alpha_{\varepsilon}\left(w_{\varepsilon}\right)\left|D^{2} u_{\varepsilon}\right|^{2}+2 \alpha_{\varepsilon}\left(w_{\varepsilon}\right) D u_{\varepsilon} \cdot D \Delta u_{\varepsilon} \\
& =2 \alpha_{\varepsilon}\left(w_{\varepsilon}\right)\left|D^{2} u_{\varepsilon}\right|^{2}+2 D u_{\varepsilon} \cdot D\left(\alpha_{\varepsilon}\left(w_{\varepsilon}\right) \Delta u_{\varepsilon}\right)-2 \alpha_{\varepsilon}^{\prime}\left(w_{\varepsilon}\right) D u_{\varepsilon} \cdot D w_{\varepsilon} \Delta u_{\varepsilon} .
\end{aligned}
$$

On the other hand, by 6.9 ) we have

$$
\alpha_{\varepsilon}\left(w_{\varepsilon}\right) \Delta u_{\varepsilon}=\lambda u_{\varepsilon}+H\left(x, D u_{\varepsilon}\right)-(p-2) \alpha_{\varepsilon}\left(w_{\varepsilon}\right) \frac{D^{2} u_{\varepsilon} D u_{\varepsilon} D u_{\varepsilon}}{\varepsilon+w_{\varepsilon}}
$$

and since last term can also be read as $D^{2} u_{\varepsilon} D u_{\varepsilon} D u_{\varepsilon}=\frac{1}{2} D w_{\varepsilon} \cdot D u_{\varepsilon}$, we have

$$
\begin{gathered}
2 D u_{\varepsilon} \cdot D\left(\alpha_{\varepsilon}\left(w_{\varepsilon}\right) \Delta u_{\varepsilon}\right)=2 D u_{\varepsilon} \cdot D\left(\lambda u_{\varepsilon}+H\left(x, D u_{\varepsilon}\right)\right) \\
-2(p-2) \alpha_{\varepsilon}^{\prime}\left(w_{\varepsilon}\right) D u_{\varepsilon} \cdot D w_{\varepsilon} \frac{D^{2} u_{\varepsilon} D u_{\varepsilon} D u_{\varepsilon}}{\varepsilon+w_{\varepsilon}} \\
+(p-2) \alpha_{\varepsilon}\left(w_{\varepsilon}\right) \frac{\left(D u_{\varepsilon} \cdot D w_{\varepsilon}\right)^{2}}{\left(\varepsilon+w_{\varepsilon}\right)^{2}}-(p-2) \frac{\alpha_{\varepsilon}\left(w_{\varepsilon}\right)}{\varepsilon+w_{\varepsilon}}\left[D^{2} w_{\varepsilon} D u_{\varepsilon} D u_{\varepsilon}+\frac{1}{2}\left|D w_{\varepsilon}\right|^{2}\right] .
\end{gathered}
$$

Thus, joining 6.12 and 6.13, we deduce

$$
\begin{gathered}
\alpha_{\varepsilon}\left(w_{\varepsilon}\right) \Delta w_{\varepsilon}=2 \alpha_{\varepsilon}\left(w_{\varepsilon}\right)\left|D^{2} u_{\varepsilon}\right|^{2}+2 D u_{\varepsilon} \cdot D\left[\lambda u_{\varepsilon}+H\left(x, D u_{\varepsilon}\right)\right] \\
-2 \alpha_{\varepsilon}^{\prime}\left(w_{\varepsilon}\right) D u_{\varepsilon} \cdot D w_{\varepsilon}\left[\mathcal{A}_{\varepsilon, u_{\varepsilon}}\left(D^{2} u_{\varepsilon}\right)\right] \\
+(p-2) \alpha_{\varepsilon}\left(w_{\varepsilon}\right) \frac{\left(D u_{\varepsilon} \cdot D w_{\varepsilon}\right)^{2}}{\left(\varepsilon+w_{\varepsilon}\right)^{2}}-(p-2) \frac{\alpha_{\varepsilon}\left(w_{\varepsilon}\right)}{\varepsilon+w_{\varepsilon}}\left[D^{2} w_{\varepsilon} D u_{\varepsilon} D u_{\varepsilon}+\frac{1}{2}\left|D w_{\varepsilon}\right|^{2}\right],
\end{gathered}
$$

and consequently

$$
\begin{gathered}
\alpha_{\varepsilon}\left(w_{\varepsilon}\right) \mathcal{A}_{\varepsilon, u_{\varepsilon}}\left(D^{2} w_{\varepsilon}\right)=2 \alpha_{\varepsilon}\left(w_{\varepsilon}\right)\left|D^{2} u_{\varepsilon}\right|^{2}+2 D u_{\varepsilon} \cdot D\left(\lambda u_{\varepsilon}+H\left(x, D u_{\varepsilon}\right)\right) \\
-2 \alpha_{\varepsilon}^{\prime}\left(w_{\varepsilon}\right) D u_{\varepsilon} \cdot D w_{\varepsilon}\left[\mathcal{A}_{\varepsilon, u_{\varepsilon}}\left(D^{2} u_{\varepsilon}\right)\right]+(p-2) \frac{\alpha_{\varepsilon}\left(w_{\varepsilon}\right)}{\varepsilon+w_{\varepsilon}}\left[\frac{\left(D u_{\varepsilon} \cdot D w_{\varepsilon}\right)^{2}}{\varepsilon+w_{\varepsilon}}-\frac{1}{2}\left|D w_{\varepsilon}\right|^{2}\right] .
\end{gathered}
$$

Computing

$$
\begin{aligned}
D u_{\varepsilon} \cdot D\left[\lambda u_{\varepsilon}\right. & \left.+H\left(x, D u_{\varepsilon}\right)\right]=\lambda w_{\varepsilon}+D u_{\varepsilon} \cdot D\left[H_{\xi}\left(x, D u_{\varepsilon}\right) D^{2} u_{\varepsilon}+H_{x}\left(x, D u_{\varepsilon}\right)\right] \\
& =\lambda w_{\varepsilon}+\frac{1}{2} D w_{\varepsilon} \cdot H_{\xi}\left(x, D u_{\varepsilon}\right)+D u_{\varepsilon} \cdot H_{x}\left(x, D u_{\varepsilon}\right)
\end{aligned}
$$

we obtain 6.11).

We refer to Proposition 6.1 as our starting point and we develop next global and local refinements to get gradient bounds.

6.1. Global gradient estimates. This subsection is devoted to the proof of Theorem 2.1, which is an adaptation to the $p$-Laplacian $(p>2)$ of the P.L. Lions's proof when $p=2$ (see for instance [29]).

In order to simplify the tedious and technical computations of the proof, we make an intermediate step. We set

$$
v_{\varepsilon}=\left(1+\beta\left(u_{\varepsilon}\right)\right)\left|D u_{\varepsilon}\right|^{2}=\left(1+\beta\left(u_{\varepsilon}\right)\right) w_{\varepsilon},
$$

where $\beta \in C^{2}(\mathbb{R})$ is a positive function, and we write the equation solved by $v_{\varepsilon}$. Then we have the following result. 
Proposition 6.2. Let $u_{\varepsilon}$ be a solution to (6.9) and $v_{\varepsilon}$ the function defined in 6.15). There exists a vector field $T_{\varepsilon} \in\left(C^{1}\right)^{N}$ such that $v_{\varepsilon}$ satisfies

$$
\begin{gathered}
\alpha_{\varepsilon}\left(w_{\varepsilon}\right) \mathcal{A}_{\varepsilon, u_{\varepsilon}}\left(D^{2} v_{\varepsilon}\right)-T_{\varepsilon} \cdot D v_{\varepsilon} \geq 2 \lambda v_{\varepsilon} \\
2\left(1+\beta\left(u_{\varepsilon}\right)\right) \alpha_{\varepsilon}\left(w_{\varepsilon}\right)\left|D^{2} u_{\varepsilon}\right|^{2}+2\left(1+\beta\left(u_{\varepsilon}\right)\right) D u_{\varepsilon} \cdot H_{x}\left(x, D u_{\varepsilon}\right) \\
+\alpha_{\varepsilon}\left(w_{\varepsilon}\right) w_{\varepsilon}^{2}\left[\beta^{\prime \prime}\left(u_{\varepsilon}\right)\left((p-1)-\frac{\varepsilon(p-2)}{\varepsilon+w_{\varepsilon}}\right)-\left(p+\frac{p-2}{2}\right) \frac{\beta^{\prime 2}\left(u_{\varepsilon}\right)}{1+\beta\left(u_{\varepsilon}\right)}\right] \\
-\beta^{\prime}\left(u_{\varepsilon}\right) w_{\varepsilon}\left\{H_{\xi}\left(x, D u_{\varepsilon}\right) \cdot D u_{\varepsilon}-\left[1+\frac{(p-2) w_{\varepsilon}}{\varepsilon+w_{\varepsilon}}\right]\left[\lambda u_{\varepsilon}+H\left(x, D u_{\varepsilon}\right)\right]\right\} \quad \text { in } \Omega .
\end{gathered}
$$

Proof. We start observing that

$$
D v_{\varepsilon}=\beta^{\prime}\left(u_{\varepsilon}\right) w_{\varepsilon} D u_{\varepsilon}+\left(1+\beta\left(u_{\varepsilon}\right)\right) D w_{\varepsilon}
$$

and

$$
\begin{gathered}
D^{2} v_{\varepsilon}=\left(1+\beta\left(u_{\varepsilon}\right)\right) D^{2} w_{\varepsilon}+\beta^{\prime}\left(u_{\varepsilon}\right)\left[D u_{\varepsilon} \otimes D w_{\varepsilon}+D w_{\varepsilon} \otimes D u_{\varepsilon}\right] \\
+\beta^{\prime \prime}\left(u_{\varepsilon}\right) w_{\varepsilon} D u_{\varepsilon} \otimes D u_{\varepsilon}+\beta^{\prime}\left(u_{\varepsilon}\right) w_{\varepsilon} D^{2} u_{\varepsilon} .
\end{gathered}
$$

Consequently

$$
\begin{aligned}
\mathcal{A}_{\varepsilon, u_{\varepsilon}}\left(D^{2} v_{\varepsilon}\right) & =\left(1+\beta\left(u_{\varepsilon}\right)\right) \mathcal{A}_{\varepsilon, u_{\varepsilon}}\left(D^{2} w_{\varepsilon}\right)+2 \beta^{\prime}\left(u_{\varepsilon}\right)\left[D w_{\varepsilon} \cdot D u_{\varepsilon}+(p-2) \frac{D w_{\varepsilon} \cdot D u_{\varepsilon}}{\varepsilon+w_{\varepsilon}}\left|D u_{\varepsilon}\right|^{2}\right] \\
& +\beta^{\prime \prime}\left(u_{\varepsilon}\right) w_{\varepsilon}\left(\left|D u_{\varepsilon}\right|^{2}+(p-2) \frac{\left|D u_{\varepsilon}\right|^{4}}{\varepsilon+w_{\varepsilon}}\right)+\beta^{\prime}\left(u_{\varepsilon}\right) w_{\varepsilon} \mathcal{A}_{\varepsilon, u_{\varepsilon}}\left(D^{2} u_{\varepsilon}\right) .
\end{aligned}
$$

We, now, exploit that

$$
D w_{\varepsilon}=\frac{D v_{\varepsilon}}{1+\beta\left(u_{\varepsilon}\right)}-w_{\varepsilon} \frac{\beta^{\prime}\left(u_{\varepsilon}\right)}{1+\beta\left(u_{\varepsilon}\right)} D u_{\varepsilon}
$$

which implies

$$
D w_{\varepsilon} \cdot D u_{\varepsilon}=\frac{D v_{\varepsilon} \cdot D u_{\varepsilon}}{1+\beta\left(u_{\varepsilon}\right)}-\frac{\beta^{\prime}\left(u_{\varepsilon}\right) w_{\varepsilon}^{2}}{1+\beta\left(u_{\varepsilon}\right)}
$$

so that:

$$
\begin{gathered}
\mathcal{A}_{\varepsilon, u_{\varepsilon}}\left(D^{2} v_{\varepsilon}\right)=\left(1+\beta\left(u_{\varepsilon}\right)\right) \mathcal{A}_{\varepsilon, u_{\varepsilon}}\left(D^{2} w_{\varepsilon}\right)+2 \frac{\beta^{\prime}\left(u_{\varepsilon}\right)}{1+\beta\left(u_{\varepsilon}\right)}\left[1+(p-2) \frac{w_{\varepsilon}}{\varepsilon+w_{\varepsilon}}\right] D v_{\varepsilon} \cdot D u_{\varepsilon} \\
-2 \frac{\beta^{\prime 2}\left(u_{\varepsilon}\right) w_{\varepsilon}^{2}}{1+\beta\left(u_{\varepsilon}\right)}\left[1+\frac{(p-2) w_{\varepsilon}}{\varepsilon+w_{\varepsilon}}\right]+\beta^{\prime \prime}\left(u_{\varepsilon}\right) w_{\varepsilon}^{2}\left[1+\frac{(p-2) w_{\varepsilon}}{\varepsilon+w_{\varepsilon}}\right]+\beta^{\prime}\left(u_{\varepsilon}\right) w_{\varepsilon} \mathcal{A}_{\varepsilon, u_{\varepsilon}}\left(D^{2} u_{\varepsilon}\right) .
\end{gathered}
$$

Thus, multiplying by $\alpha_{\varepsilon}\left(w_{\varepsilon}\right)$, we deduce:

$$
\begin{gathered}
\alpha_{\varepsilon}\left(w_{\varepsilon}\right) \mathcal{A}_{\varepsilon, u_{\varepsilon}}\left(D^{2} v_{\varepsilon}\right)=2 \alpha_{\varepsilon}\left(w_{\varepsilon}\right) \frac{\beta^{\prime}\left(u_{\varepsilon}\right)}{1+\beta\left(u_{\varepsilon}\right)}\left[1+(p-2) \frac{w_{\varepsilon}}{\varepsilon+w_{\varepsilon}}\right] D v_{\varepsilon} \cdot D u_{\varepsilon} \\
+\alpha_{\varepsilon}\left(w_{\varepsilon}\right) w_{\varepsilon}^{2}\left[\beta^{\prime \prime}\left(u_{\varepsilon}\right)-2 \frac{\beta^{\prime 2}\left(u_{\varepsilon}\right)}{1+\beta\left(u_{\varepsilon}\right)}\right]\left[1+(p-2) \frac{w_{\varepsilon}}{\varepsilon+w_{\varepsilon}}\right] \\
+\left(1+\beta\left(u_{\varepsilon}\right)\right) \alpha_{\varepsilon}\left(w_{\varepsilon}\right) \mathcal{A}_{\varepsilon, u_{\varepsilon}}\left(D^{2} w_{\varepsilon}\right)+\beta^{\prime}\left(u_{\varepsilon}\right) w_{\varepsilon}\left[\alpha_{\varepsilon}\left(w_{\varepsilon}\right) \mathcal{A}_{\varepsilon, u_{\varepsilon}}\left(D^{2} u_{\varepsilon}\right)\right] .
\end{gathered}
$$

Using now (6.11) we obtain

$$
\begin{gathered}
\alpha_{\varepsilon}\left(w_{\varepsilon}\right) \mathcal{A}_{\varepsilon, u_{\varepsilon}}\left(D^{2} v_{\varepsilon}\right)=2 \alpha_{\varepsilon}\left(w_{\varepsilon}\right) \frac{\beta^{\prime}\left(u_{\varepsilon}\right)}{1+\beta\left(u_{\varepsilon}\right)}\left[1+(p-2) \frac{w_{\varepsilon}}{\varepsilon+w_{\varepsilon}}\right] D v_{\varepsilon} \cdot D u_{\varepsilon} \\
+\alpha_{\varepsilon}\left(w_{\varepsilon}\right) w_{\varepsilon}^{2}\left[\beta^{\prime \prime}\left(u_{\varepsilon}\right)-2 \frac{\beta^{\prime 2}\left(u_{\varepsilon}\right)}{1+\beta\left(u_{\varepsilon}\right)}\right]\left[1+(p-2) \frac{w_{\varepsilon}}{\varepsilon+w_{\varepsilon}}\right] \\
\left(1+\beta\left(u_{\varepsilon}\right)\right)\left\{2 \lambda w_{\varepsilon}+2 \alpha_{\varepsilon}\left(w_{\varepsilon}\right)\left|D^{2} u_{\varepsilon}\right|^{2}+2 D u_{\varepsilon} \cdot H_{x}\left(x, D u_{\varepsilon}\right)\right. \\
+H_{\xi}\left(x, D u_{\varepsilon}\right) \cdot D w_{\varepsilon}-2 \alpha_{\varepsilon}^{\prime}\left(w_{\varepsilon}\right) \mathcal{A}_{\varepsilon, u_{\varepsilon}}\left(D^{2} u_{\varepsilon}\right) D u_{\varepsilon} D w_{\varepsilon} \\
\left.+(p-2) \frac{\alpha_{\varepsilon}\left(w_{\varepsilon}\right)}{\left(\varepsilon+w_{\varepsilon}\right)^{2}}\left(D u_{\varepsilon} \cdot D w_{\varepsilon}\right)^{2}-\frac{p-2}{2} \frac{\alpha_{\varepsilon}\left(w_{\varepsilon}\right)}{\varepsilon+w_{\varepsilon}}\left|D w_{\varepsilon}\right|^{2}\right\} \\
+\beta^{\prime}\left(u_{\varepsilon}\right) w_{\varepsilon} \alpha_{\varepsilon}\left(w_{\varepsilon}\right) \mathcal{A}_{\varepsilon, u_{\varepsilon}}\left(D^{2} u_{\varepsilon}\right) .
\end{gathered}
$$


Hence by 6.17 and 6.18 we deduce that there exists a vector field $T_{\varepsilon}$ such that (6.19)

$$
\begin{gathered}
\alpha_{\varepsilon}\left(w_{\varepsilon}\right) \mathcal{A}_{\varepsilon, u_{\varepsilon}}\left(D^{2} v_{\varepsilon}\right)-T_{\varepsilon} \cdot D v_{\varepsilon} \\
=\alpha_{\varepsilon}\left(w_{\varepsilon}\right) w_{\varepsilon}^{2}\left[\beta^{\prime \prime}\left(u_{\varepsilon}\right)-2 \frac{\beta^{\prime 2}\left(u_{\varepsilon}\right)}{1+\beta\left(u_{\varepsilon}\right)}\right]\left[1+(p-2) \frac{w_{\varepsilon}}{\varepsilon+w_{\varepsilon}}\right] \\
+\left(1+\beta\left(u_{\varepsilon}\right)\right)\left\{2 \lambda w_{\varepsilon}+2 \alpha_{\varepsilon}\left(w_{\varepsilon}\right)\left|D^{2} u_{\varepsilon}\right|^{2}+2 D u_{\varepsilon} \cdot H_{x}\left(x, D u_{\varepsilon}\right)\right\}-\beta^{\prime}\left(u_{\varepsilon}\right) w_{\varepsilon} H_{\xi}\left(x, D u_{\varepsilon}\right) \cdot D u_{\varepsilon} \\
+(p-2) \frac{\alpha_{\varepsilon}\left(w_{\varepsilon}\right)}{\left(\varepsilon+w_{\varepsilon}\right)^{2}} \frac{\beta^{\prime 2}\left(u_{\varepsilon}\right) w_{\varepsilon}^{4}}{1+\beta\left(u_{\varepsilon}\right)}-\frac{p-2}{2} \frac{\alpha_{\varepsilon}\left(w_{\varepsilon}\right)}{\varepsilon+w_{\varepsilon}} w_{\varepsilon}^{2} \frac{\beta^{\prime 2}\left(u_{\varepsilon}\right)}{1+\beta\left(u_{\varepsilon}\right)}\left|D u_{\varepsilon}\right|^{2} \\
+\beta^{\prime}\left(u_{\varepsilon}\right) w_{\varepsilon}\left[1+2 w_{\varepsilon} \frac{\alpha_{\varepsilon}^{\prime}\left(w_{\varepsilon}\right)}{\alpha_{\varepsilon}\left(w_{\varepsilon}\right)}\right]\left[\alpha_{\varepsilon}\left(w_{\varepsilon}\right) \mathcal{A}_{\varepsilon, u_{\varepsilon}}\left(D^{2} u_{\varepsilon}\right)\right] .
\end{gathered}
$$

Recall that $w_{\varepsilon}=\left|D u_{\varepsilon}\right|^{2}$; so we have

$$
\begin{gathered}
(p-2) \frac{\alpha_{\varepsilon}\left(w_{\varepsilon}\right)}{\left(\varepsilon+w_{\varepsilon}\right)^{2}} \frac{\beta^{\prime 2}\left(u_{\varepsilon}\right) w_{\varepsilon}^{4}}{1+\beta\left(u_{\varepsilon}\right)}-\frac{p-2}{2} \frac{\alpha_{\varepsilon}\left(w_{\varepsilon}\right)}{\varepsilon+w_{\varepsilon}} w_{\varepsilon}^{2} \frac{\beta^{\prime 2}\left(u_{\varepsilon}\right)}{1+\beta\left(u_{\varepsilon}\right)}\left|D u_{\varepsilon}\right|^{2} \\
\geq \alpha_{\varepsilon}\left(w_{\varepsilon}\right) w_{\varepsilon}^{2} \frac{\beta^{\prime 2}\left(u_{\varepsilon}\right)}{1+\beta\left(u_{\varepsilon}\right)}\left[\frac{p-2}{2}-\frac{3}{2} \frac{\varepsilon}{\varepsilon+w_{\varepsilon}}\right] .
\end{gathered}
$$

Therefore, putting together the second and fourth line from 6.19 we deduce

$$
\begin{gathered}
\alpha_{\varepsilon}\left(w_{\varepsilon}\right) \mathcal{A}_{\varepsilon, u_{\varepsilon}}\left(D^{2} v_{\varepsilon}\right)-T_{\varepsilon} \cdot D v_{\varepsilon} \\
\geq \alpha_{\varepsilon}\left(w_{\varepsilon}\right) w_{\varepsilon}^{2}\left[\beta^{\prime \prime}\left(u_{\varepsilon}\right)\left((p-1)-\frac{\varepsilon(p-2)}{\varepsilon+w_{\varepsilon}}\right)-\left(p+\frac{p-2}{2}\right) \frac{\beta^{\prime 2}\left(u_{\varepsilon}\right)}{1+\beta\left(u_{\varepsilon}\right)}\right] \\
+\left(1+\beta\left(u_{\varepsilon}\right)\right)\left\{2 \lambda w_{\varepsilon}+2 \alpha_{\varepsilon}\left(w_{\varepsilon}\right)\left|D^{2} u_{\varepsilon}\right|^{2}+2 D u_{\varepsilon} \cdot H_{x}\left(x, D u_{\varepsilon}\right)\right\}-\beta^{\prime}\left(u_{\varepsilon}\right) w_{\varepsilon} H_{\xi}\left(x, D u_{\varepsilon}\right) \cdot D u_{\varepsilon} \\
+\beta^{\prime}\left(u_{\varepsilon}\right) w_{\varepsilon}\left[1+2 w_{\varepsilon} \frac{\alpha_{\varepsilon}^{\prime}\left(w_{\varepsilon}\right)}{\alpha_{\varepsilon}\left(w_{\varepsilon}\right)}\right]\left[\alpha_{\varepsilon}\left(w_{\varepsilon}\right) \mathcal{A}_{\varepsilon, u_{\varepsilon}}\left(D^{2} u_{\varepsilon}\right)\right] .
\end{gathered}
$$

Since $\frac{\alpha_{\varepsilon}^{\prime}(s)}{\alpha_{\varepsilon}(s)}=\frac{p-2}{2} \frac{1}{\varepsilon+s}$, and using the equation 6.9 solved by $u_{\varepsilon}$, we transform the last term and we get

$$
\begin{gathered}
\alpha_{\varepsilon}\left(w_{\varepsilon}\right) \mathcal{A}_{\varepsilon, u_{\varepsilon}}\left(D^{2} v_{\varepsilon}\right)-T_{\varepsilon} \cdot D v_{\varepsilon} \\
\geq \alpha_{\varepsilon}\left(w_{\varepsilon}\right) w_{\varepsilon}^{2}\left[\beta^{\prime \prime}\left(u_{\varepsilon}\right)\left((p-1)-\frac{\varepsilon(p-2)}{\varepsilon+w_{\varepsilon}}\right)-\left(p+\frac{p-2}{2}\right) \frac{\beta^{\prime 2}\left(u_{\varepsilon}\right)}{1+\beta\left(u_{\varepsilon}\right)}\right] \\
+\left(1+\beta\left(u_{\varepsilon}\right)\right)\left\{2 \lambda w_{\varepsilon}+2 \alpha_{\varepsilon}\left(w_{\varepsilon}\right)\left|D^{2} u_{\varepsilon}\right|^{2}+2 D u_{\varepsilon} \cdot H_{x}\left(x, D u_{\varepsilon}\right)\right\} \\
-\beta^{\prime}\left(u_{\varepsilon}\right) w_{\varepsilon} H_{\xi}\left(x, D u_{\varepsilon}\right) \cdot D u_{\varepsilon}+\beta^{\prime}\left(u_{\varepsilon}\right) w_{\varepsilon}\left[1+\frac{(p-2) w_{\varepsilon}}{\varepsilon+w_{\varepsilon}}\right]\left[\lambda u_{\varepsilon}+H\left(x, D u_{\varepsilon}\right)\right] .
\end{gathered}
$$

from which we deduce 6.16 .

Using the above Proposition we are now ready to prove Theorem 2.1 .

Proof of Theorem 2.1. From 6.16 we deduce

$$
\begin{gathered}
\alpha_{\varepsilon}\left(w_{\varepsilon}\right) \mathcal{A}_{\varepsilon, u_{\varepsilon}}\left(D^{2} v_{\varepsilon}\right)-T_{\varepsilon} \cdot D v_{\varepsilon} \geq 2 \lambda v_{\varepsilon} \\
+2\left(1+\beta\left(u_{\varepsilon}\right)\right) \alpha_{\varepsilon}\left(w_{\varepsilon}\right)\left|D^{2} u_{\varepsilon}\right|^{2}+2\left(1+\beta\left(u_{\varepsilon}\right)\right) D u_{\varepsilon} \cdot H_{x}\left(x, D u_{\varepsilon}\right) \\
+\alpha_{\varepsilon}\left(w_{\varepsilon}\right) w_{\varepsilon}^{2}\left[\beta^{\prime \prime}\left(u_{\varepsilon}\right)\left((p-1)-\frac{\varepsilon(p-2)}{\varepsilon+w_{\varepsilon}}\right)-\left(p+\frac{p-2}{2}\right) \frac{\beta^{\prime 2}\left(u_{\varepsilon}\right)}{1+\beta\left(u_{\varepsilon}\right)}\right] \\
-\beta^{\prime}\left(u_{\varepsilon}\right) w_{\varepsilon}\left\{H_{\xi}\left(x, D u_{\varepsilon}\right) \cdot D u_{\varepsilon}-(p-1) H\left(x, D u_{\varepsilon}\right)\right\} \\
-(p-1)\left|\beta^{\prime}\left(u_{\varepsilon}\right)\right| w_{\varepsilon}\left\|\lambda u_{\varepsilon}\right\|_{L^{\infty}(\Omega)}-\varepsilon(p-2)\left|\beta^{\prime}\left(u_{\varepsilon}\right)\right| \frac{w_{\varepsilon}}{\varepsilon+w_{\varepsilon}} H\left(x, D u_{\varepsilon}\right) .
\end{gathered}
$$


We start by noticing that

$$
\frac{1}{\sqrt{N}}\left|\Delta u_{\varepsilon}\right| \leq\left|D^{2} u_{\varepsilon}\right| \quad \text { and } \quad\left|\frac{D^{2} u_{\varepsilon} D u_{\varepsilon} D u_{\varepsilon}}{\varepsilon+\left|D u_{\varepsilon}\right|^{2}}\right| \leq\left|D^{2} u_{\varepsilon}\right|
$$

and consequently

$$
\left|D^{2} u_{\varepsilon}\right| \geq \eta\left|\mathcal{A}_{\varepsilon, u_{\varepsilon}}\left(D^{2} u_{\varepsilon}\right)\right|, \quad \text { where } \quad \eta=\frac{1}{|p-2|+\sqrt{N}},
$$

that implies, using the definition of $\alpha_{\varepsilon}$ and the equation of $u_{\varepsilon}$,

$$
\begin{gathered}
2\left(1+\beta\left(u_{\varepsilon}\right)\right) \alpha_{\varepsilon}\left(w_{\varepsilon}\right)\left|D^{2} u_{\varepsilon}\right|^{2} \geq 2\left(1+\beta\left(u_{\varepsilon}\right)\right) \eta^{2} \frac{\left[\alpha_{\varepsilon}\left(w_{\varepsilon}\right) \mathcal{A}_{\varepsilon, u_{\varepsilon}}\left(D^{2} u_{\varepsilon}\right)\right]^{2}}{\alpha_{\varepsilon}\left(w_{\varepsilon}\right)} \\
\quad \geq 2\left(1+\beta\left(u_{\varepsilon}\right)\right) \eta^{2} \frac{\left|D u_{\varepsilon}\right|^{2-p}}{(1+\varepsilon)^{\frac{p-2}{2}}}\left[\lambda u_{\varepsilon}+H\left(x, D u_{\varepsilon}\right)\right]^{2} \chi_{\left\{\left|D u_{\varepsilon}\right| \geq 1\right\}} .
\end{gathered}
$$

In particular, for every $\theta \in(0,1)$ we have

$$
\begin{gathered}
2\left(1+\beta\left(u_{\varepsilon}\right)\right) \alpha_{\varepsilon}\left(w_{\varepsilon}\right)\left|D^{2} u_{\varepsilon}\right|^{2}-\varepsilon(p-2)\left|\beta^{\prime}\left(u_{\varepsilon}\right)\right| \frac{w_{\varepsilon}}{\varepsilon+w_{\varepsilon}} H\left(x, D u_{\varepsilon}\right) \\
\geq 2\left(1+\beta\left(u_{\varepsilon}\right)\right) \eta^{2}(1-\theta)\left|D u_{\varepsilon}\right|^{2-p} H^{2}\left(x, D u_{\varepsilon}\right) \\
-c\left(1+\beta\left(u_{\varepsilon}\right)\right)\left\|\lambda u_{\varepsilon}\right\|_{L^{\infty}(\Omega)}^{2}-\varepsilon c \frac{\left|\beta^{\prime}\left(u_{\varepsilon}\right)\right|^{2}}{1+\beta\left(u_{\varepsilon}\right)}\left|D u_{\varepsilon}\right|^{p-2} .
\end{gathered}
$$

Thus, from 6.20 we deduce

$$
\begin{gathered}
\alpha_{\varepsilon}\left(w_{\varepsilon}\right) \mathcal{A}_{\varepsilon, u_{\varepsilon}}\left(D^{2} v_{\varepsilon}\right)-T_{\varepsilon} \cdot D v_{\varepsilon} \geq 2 \lambda v_{\varepsilon} \\
+2\left(1+\beta\left(u_{\varepsilon}\right)\right)(1-\theta) \eta^{2}\left|D u_{\varepsilon}\right|^{2-p} H^{2}\left(x, D u_{\varepsilon}\right)+2\left(1+\beta\left(u_{\varepsilon}\right)\right) D u_{\varepsilon} \cdot H_{x}\left(x, D u_{\varepsilon}\right) \\
+\alpha_{\varepsilon}\left(w_{\varepsilon}\right) w_{\varepsilon}^{2}\left[\beta^{\prime \prime}\left(u_{\varepsilon}\right)\left((p-1)-\frac{\varepsilon(p-2)}{\varepsilon+w_{\varepsilon}}\right)-\left(p+\frac{p-2}{2}\right) \frac{\beta^{\prime 2}\left(u_{\varepsilon}\right)}{1+\beta\left(u_{\varepsilon}\right)}\right] \\
-\beta^{\prime}\left(u_{\varepsilon}\right) w_{\varepsilon}\left[D u_{\varepsilon} \cdot H_{\xi}\left(x, D u_{\varepsilon}\right)-(p-1) H\left(x, D u_{\varepsilon}\right)\right] \\
-(p-1)\left|\beta^{\prime}\left(u_{\varepsilon}\right)\right| w_{\varepsilon}\left\|\lambda u_{\varepsilon}\right\|_{L^{\infty}(\Omega)}-c\left(1+\beta\left(u_{\varepsilon}\right)\right)\left\|\lambda u_{\varepsilon}\right\|_{L^{\infty}(\Omega)}^{2}-\varepsilon c \frac{\left|\beta^{\prime}\left(u_{\varepsilon}\right)\right|^{2}}{1+\beta\left(u_{\varepsilon}\right)}\left|D u_{\varepsilon}\right|^{p-2},
\end{gathered}
$$

Suppose that 2.5 holds with $\tau=1$; then we set the function $\beta$ in the following way

$$
\beta(s)=\mu e^{(M-s)^{2}}
$$

with $\mu, M \in \mathbb{R}^{+}$. The case $\tau=-1$ can be handled similarly by considering $\beta(s)=\mu e^{(M+s)^{2}}$.

First we rewrite last two lines of 6.21), which becomes

$$
\begin{gathered}
\alpha_{\varepsilon}\left(w_{\varepsilon}\right) \mathcal{A}_{\varepsilon, u_{\varepsilon}}\left(D^{2} v_{\varepsilon}\right)-T_{\varepsilon} \cdot D v_{\varepsilon} \geq 2 \lambda v_{\varepsilon} \\
+2\left(1+\beta\left(u_{\varepsilon}\right)\right)(1-\theta) \eta^{2}\left|D u_{\varepsilon}\right|^{2-p} H^{2}\left(x, D u_{\varepsilon}\right)+2\left(1+\beta\left(u_{\varepsilon}\right)\right) D u_{\varepsilon} \cdot H_{x}\left(x, D u_{\varepsilon}\right) \\
+\alpha_{\varepsilon}\left(w_{\varepsilon}\right) w_{\varepsilon}^{2}\left[\beta^{\prime \prime}\left(u_{\varepsilon}\right)\left((p-1)-\frac{\varepsilon(p-2)}{\varepsilon+w_{\varepsilon}}\right)-\left(p+\frac{p-2}{2}\right) \frac{\beta^{\prime 2}\left(u_{\varepsilon}\right)}{1+\beta\left(u_{\varepsilon}\right)}\right] \\
+2 \mu e^{\left(M-u_{\varepsilon}\right)^{2}}\left(M-u_{\varepsilon}\right) w_{\varepsilon}\left[D u_{\varepsilon} \cdot H_{\xi}\left(x, D u_{\varepsilon}\right)-(p-1) H\left(x, D u_{\varepsilon}\right)\right. \\
\left.-(p-1)\left\|\lambda u_{\varepsilon}\right\|_{L^{\infty}(\Omega)}-\varepsilon c \frac{\left|\beta^{\prime}\left(u_{\varepsilon}\right)\right|}{1+\beta\left(u_{\varepsilon}\right)}\left|D u_{\varepsilon}\right|^{p-2}\right]-c\left(1+\beta\left(u_{\varepsilon}\right)\right)\left\|\lambda u_{\varepsilon}\right\|_{L^{\infty}(\Omega)}^{2} .
\end{gathered}
$$

We observe that, by choosing $M$ sufficiently large and subsequently $\mu$ small enough (both depending on $\left.\left\|u_{\varepsilon}\right\|_{L^{\infty}(\Omega)}\right)$, the quantity

$$
\frac{1}{\left|\beta^{\prime}\left(u_{\varepsilon}\right)\right|}\left\{\beta^{\prime \prime}\left(u_{\varepsilon}\right)\left((p-1)-\frac{\varepsilon(p-2)}{\varepsilon+w_{\varepsilon}}\right)-\left(p+\frac{p-2}{2}\right) \frac{\beta^{\prime 2}\left(u_{\varepsilon}\right)}{1+\beta\left(u_{\varepsilon}\right)}\right\}
$$


can be arbitrarily large. In particular, for any $\rho$ we can fix $M$ and $\mu$ such that

$$
\begin{aligned}
& \alpha_{\varepsilon}\left(w_{\varepsilon}\right) w_{\varepsilon}^{2}\left[\beta^{\prime \prime}\left(u_{\varepsilon}\right)\left((p-1)-\frac{\varepsilon(p-2)}{\varepsilon+w_{\varepsilon}}\right)-\left(p+\frac{p-2}{2}\right) \frac{\beta^{\prime 2}\left(u_{\varepsilon}\right)}{1+\beta\left(u_{\varepsilon}\right)}\right] \\
& >2 \rho\left|\beta^{\prime}\left(u_{\varepsilon}\right)\right| w_{\varepsilon}\left|D u_{\varepsilon}\right|^{p}=4 \rho \mu\left(M-u_{\varepsilon}\right) e^{\left(M-u_{\varepsilon}\right)^{2}} w_{\varepsilon}\left|D u_{\varepsilon}\right|^{p} .
\end{aligned}
$$

So we obtain from 6.23

$$
\begin{gathered}
\alpha_{\varepsilon}\left(w_{\varepsilon}\right) \mathcal{A}_{\varepsilon, u_{\varepsilon}}\left(D^{2} v_{\varepsilon}\right)-T_{\varepsilon} \cdot D v_{\varepsilon} \geq 2 \lambda v_{\varepsilon} \\
+2\left(1+\beta\left(u_{\varepsilon}\right)\right)\left\{(1-\theta) \eta^{2}\left|D u_{\varepsilon}\right|^{2-p} H^{2}\left(x, D u_{\varepsilon}\right)+D u_{\varepsilon} \cdot H_{x}\left(x, D u_{\varepsilon}\right)\right\} \\
+2 \mu e^{\left(M-u_{\varepsilon}\right)^{2}}\left(M-u_{\varepsilon}\right) w_{\varepsilon}\left[\rho\left|D u_{\varepsilon}\right|^{p}+D u_{\varepsilon} \cdot H_{\xi}\left(x, D u_{\varepsilon}\right)-(p-1) H\left(x, D u_{\varepsilon}\right)\right. \\
\left.-(p-1)\left\|\lambda u_{\varepsilon}\right\|_{L^{\infty}(\Omega)}-c\right]-c\left(1+\beta\left(u_{\varepsilon}\right)\right)\left\|\lambda u_{\varepsilon}\right\|_{L^{\infty}(\Omega)}^{2},
\end{gathered}
$$

provided $M$ and $\mu$ are suitably chosen. Because of (2.3), this implies that, for some constants $c_{0}, c_{1}, c_{2}$ (depending also on $\left\|\lambda u_{\varepsilon}\right\|_{L^{\infty}(\Omega)}$ ), we have

$$
\begin{gathered}
\alpha_{\varepsilon}\left(w_{\varepsilon}\right) \mathcal{A}_{\varepsilon, u_{\varepsilon}}\left(D^{2} v_{\varepsilon}\right)-T_{\varepsilon} \cdot D v_{\varepsilon} \geq 2 \lambda v_{\varepsilon} \\
+2\left(1+\beta\left(u_{\varepsilon}\right)\right)\left\{(1-\theta) \eta^{2}\left|D u_{\varepsilon}\right|^{2-p} H^{2}\left(x, D u_{\varepsilon}\right)+D u_{\varepsilon} \cdot H_{x}\left(x, D u_{\varepsilon}\right)\right. \\
\left.+c_{0} \mu\left|D u_{\varepsilon}\right|^{2}\left[\rho\left|D u_{\varepsilon}\right|^{p}+D u_{\varepsilon} \cdot H_{\xi}\left(x, D u_{\varepsilon}\right)-(p-1) H\left(x, D u_{\varepsilon}\right)-c_{1}\right]\right\}-c_{2}\left(1+\beta\left(u_{\varepsilon}\right)\right) .
\end{gathered}
$$

Thanks to (2.3)-2.4 (which imply (2.5)), choosing $\mu$ smaller if needed we deduce from the above inequality a uniform bound on the interior maxima of $v_{\varepsilon}$, through the maximum principle. Hence the conclusion follows.

6.2. Local gradient estimates. The goal of this subsection is to prove the local estimate stated in Theorem 2.5. The proof will be based on two results: first we provide a local version of Proposition 6.1, secondly we build a suitable family of cut-off functions.

Proposition 6.3. Assume that $H(x, \xi)$ satisfies (2.2) and let $u_{\varepsilon}$ be a solution of (6.9). Let $\varphi$ be any $W^{2, \infty}(\Omega)$ function with compact support in $\Omega$, and define

$$
z_{\varepsilon}=w_{\varepsilon} \varphi=\left|D u_{\varepsilon}\right|^{2} \varphi .
$$

Then there exists a $C^{1}(\Omega)^{N}$ vector field $T_{\varepsilon}$ such that

$$
\begin{gathered}
\alpha_{\varepsilon}\left(w_{\varepsilon}\right) \mathcal{A}_{\varepsilon, u_{\varepsilon}}\left(D^{2} z_{\varepsilon}\right)-T_{\varepsilon} \cdot D z_{\varepsilon}-2 \lambda z_{\varepsilon} \\
=2 \varphi \alpha_{\varepsilon}\left(w_{\varepsilon}\right)\left|D^{2} u_{\varepsilon}\right|^{2}+w_{\varepsilon} \alpha_{\varepsilon}\left(w_{\varepsilon}\right)\left[\mathcal{A}_{\varepsilon, u_{\varepsilon}}\left(D^{2} \varphi\right)-2 \mathcal{A}_{\varepsilon, u_{\varepsilon}}\left(\frac{D \varphi \otimes D \varphi}{\varphi}\right)\right] \\
+2 \varphi D u_{\varepsilon} \cdot H_{x}\left(x, D u_{\varepsilon}\right)+(p-2) \frac{w_{\varepsilon}}{\varepsilon+w_{\varepsilon}} D u_{\varepsilon} \cdot D \varphi\left[\lambda u_{\varepsilon}+H\left(x, D u_{\varepsilon}\right)\right] \\
-w_{\varepsilon} D \varphi \cdot H_{\xi}\left(x, D u_{\varepsilon}\right)-\frac{p-2}{2} \frac{\alpha_{\varepsilon}\left(w_{\varepsilon}\right) w_{\varepsilon}^{2}}{\varphi\left(\varepsilon+w_{\varepsilon}\right)}\left[\frac{2\left(D u_{\varepsilon} \cdot D \varphi\right)^{2}}{\varepsilon+w_{\varepsilon}}-|D \varphi|^{2}\right] \quad \text { in } \operatorname{supp}(\varphi) .
\end{gathered}
$$

Proof. Let us define $z_{\varepsilon}=w_{\varepsilon} \varphi$ where $\varphi \in W^{2, \infty}(\Omega)$ has compact support; noticing that

$$
D z_{\varepsilon}=w_{\varepsilon} D \varphi+\varphi D w_{\varepsilon}
$$

and

$$
D^{2} z_{\varepsilon}=w_{\varepsilon} D^{2} \varphi+\varphi D^{2} w_{\varepsilon}+D w_{\varepsilon} \otimes D \varphi+D \varphi \otimes D w_{\varepsilon},
$$

we deduce, being $\mathcal{A}_{\varepsilon, u_{\varepsilon}}(M)=\mathcal{A}_{\varepsilon, u_{\varepsilon}}\left(M^{T}\right)$ for any $M \in \mathcal{M}^{N \times N}$ and using 6.25, that

$$
\begin{aligned}
& \mathcal{A}_{\varepsilon, u_{\varepsilon}}\left(D^{2} z_{\varepsilon}\right)=\varphi \mathcal{A}_{\varepsilon, u_{\varepsilon}}\left(D^{2} w_{\varepsilon}\right)+w_{\varepsilon} \mathcal{A}_{\varepsilon, u_{\varepsilon}}\left(D^{2} \varphi\right)+2 \mathcal{A}_{\varepsilon, u_{\varepsilon}}\left(D \varphi \otimes D w_{\varepsilon}\right) \\
= & \varphi \mathcal{A}_{\varepsilon, u_{\varepsilon}}\left(D^{2} w_{\varepsilon}\right)+w_{\varepsilon} \mathcal{A}_{\varepsilon, u_{\varepsilon}}\left(D^{2} \varphi\right)+2 \mathcal{A}_{\varepsilon, u_{\varepsilon}}\left(\frac{D \varphi \otimes D z_{\varepsilon}}{\varphi}\right)-2 w_{\varepsilon} \mathcal{A}_{\varepsilon, u_{\varepsilon}}\left(\frac{D \varphi \otimes D \varphi}{\varphi}\right)
\end{aligned}
$$


which implies

$$
\begin{aligned}
& \alpha_{\varepsilon}\left(w_{\varepsilon}\right) \mathcal{A}_{\varepsilon, u_{\varepsilon}}\left(D^{2} z_{\varepsilon}\right)-2 \alpha_{\varepsilon}\left(w_{\varepsilon}\right) \mathcal{A}_{\varepsilon, u_{\varepsilon}}\left(\frac{D \varphi \otimes D z_{\varepsilon}}{\varphi}\right)=\varphi \alpha_{\varepsilon}\left(w_{\varepsilon}\right) \mathcal{A}_{\varepsilon, u_{\varepsilon}}\left(D^{2} w_{\varepsilon}\right) \\
& \quad+w_{\varepsilon} \alpha_{\varepsilon}\left(w_{\varepsilon}\right)\left[\mathcal{A}_{\varepsilon, u_{\varepsilon}}\left(D^{2} \varphi\right)-2 \mathcal{A}_{\varepsilon, u_{\varepsilon}}\left(\frac{D \varphi \otimes D \varphi}{\varphi}\right)\right] \quad \text { in } \operatorname{supp}(\varphi) .
\end{aligned}
$$

Let us concentrate on the first term in the right hand side above: using the identity 6.11) and by (6.25), we deduce that

$$
\begin{gathered}
\varphi \alpha_{\varepsilon}\left(w_{\varepsilon}\right) \mathcal{A}_{\varepsilon, u_{\varepsilon}}\left(D^{2} w_{\varepsilon}\right)=\left[H_{\xi}\left(x, D u_{\varepsilon}\right)-2 \alpha_{\varepsilon}^{\prime}\left(w_{\varepsilon}\right) \mathcal{A}_{\varepsilon, u_{\varepsilon}}\left(D^{2} u_{\varepsilon}\right) D u_{\varepsilon}\right] \cdot D z_{\varepsilon} \\
-w_{\varepsilon}\left[H_{\xi}\left(x, D u_{\varepsilon}\right)-2 \alpha_{\varepsilon}^{\prime}\left(w_{\varepsilon}\right) \mathcal{A}_{\varepsilon, u_{\varepsilon}}\left(D^{2} u_{\varepsilon}\right) D u_{\varepsilon}\right] \cdot D \varphi \\
+2 \lambda \varphi w_{\varepsilon}+2 \varphi \alpha_{\varepsilon}\left(w_{\varepsilon}\right)\left|D^{2} u_{\varepsilon}\right|^{2}+2 \varphi D u_{\varepsilon} \cdot H_{x}\left(x, D u_{\varepsilon}\right) \\
+(p-2) \frac{\alpha_{\varepsilon}\left(w_{\varepsilon}\right)}{\varphi\left(\varepsilon+w_{\varepsilon}\right)}\left[\frac{\left(D u_{\varepsilon} \cdot D z_{\varepsilon}-w_{\varepsilon} D u_{\varepsilon} \cdot D \varphi\right)^{2}}{\varepsilon+w_{\varepsilon}}-\frac{1}{2}\left|D z_{\varepsilon}-w_{\varepsilon} D \varphi\right|^{2}\right] .
\end{gathered}
$$

We develop the squares of the last line so that, joining the new identity with (6.26), we deduce that there exists a vector field $T_{\varepsilon}$ such that the following equation is solved by $z_{\varepsilon}$ in $\operatorname{supp}(\varphi)$ :

$$
\begin{gathered}
\alpha_{\varepsilon}\left(w_{\varepsilon}\right) \mathcal{A}_{\varepsilon, u_{\varepsilon}}\left(D^{2} z_{\varepsilon}\right)-T_{\varepsilon} \cdot D z_{\varepsilon}-2 \lambda z_{\varepsilon} \\
=w_{\varepsilon} \alpha_{\varepsilon}\left(w_{\varepsilon}\right)\left[\mathcal{A}_{\varepsilon, u_{\varepsilon}}\left(D^{2} \varphi\right)-2 \mathcal{A}_{\varepsilon, u_{\varepsilon}}\left(\frac{D \varphi \otimes D \varphi}{\varphi}\right)\right] \\
+2 \varphi \alpha_{\varepsilon}\left(w_{\varepsilon}\right)\left|D^{2} u_{\varepsilon}\right|^{2}+2 \varphi D u_{\varepsilon} \cdot H_{x}\left(x, D u_{\varepsilon}\right)-w_{\varepsilon} H_{\xi}\left(x, D u_{\varepsilon}\right) \cdot D \varphi \\
-2 \alpha_{\varepsilon}^{\prime}\left(w_{\varepsilon}\right) \mathcal{A}_{\varepsilon, u_{\varepsilon}}\left(D^{2} u_{\varepsilon}\right) D u_{\varepsilon} \cdot D \varphi \\
+(p-2) \frac{\alpha_{\varepsilon}\left(w_{\varepsilon}\right) w_{\varepsilon}^{2}}{\varphi\left(\varepsilon+w_{\varepsilon}\right)}\left[\frac{\left(D u_{\varepsilon} \cdot D \varphi\right)^{2}}{\varepsilon+w_{\varepsilon}}-\frac{|D \varphi|^{2}}{2}\right] .
\end{gathered}
$$

Since $2 \alpha_{\varepsilon}^{\prime}(s)=\frac{\alpha_{\varepsilon}(s)}{s+\varepsilon}$, using the equation solved by $u_{\varepsilon}$ (i.e. 6.9 ) we conclude with 6.24).

The next result fixes the choice of the cut-off function $\varphi$. Indeed, let $\varsigma$ be any $W^{2, \infty}(\Omega)$ (we can suppose without loss of generality that $|\varsigma| \leq 1)$ such that $\operatorname{supp}(\varsigma) \subseteq \omega^{\prime}, \omega^{\prime} \subset \subset \Omega$. Then we can choose $\varphi=\varphi(\varsigma)$ in the following way.

Lemma 6.4. Assume that (2.10)-2.11) hold true and let $\varsigma$ be any $W^{2, \infty}(\Omega)$ such that $\operatorname{supp}(\varsigma) \subseteq$ $\omega^{\prime} \subset \subset \Omega$ and $0 \leq \varsigma \leq 1$. Then, for any $\delta>0$, there exists a constant $K_{\delta}>0$ and a function $\varphi:[0,1] \rightarrow[0,1], \varphi \in C^{0}([0,1]) \cap C^{2}(0,1)$ with $\varphi(0)=\varphi^{\prime}(0)=0, \varphi(1)=1$, and such that the following inequality holds true

$$
t^{2}\left(t^{2}+\varepsilon\right)^{\frac{p-2}{2}}\left(\frac{|D \varphi(\varsigma)|^{2}}{\varphi(\varsigma)}+\left|D^{2} \varphi(\varsigma)\right|\right) \leq \frac{\kappa}{\left(t^{2}+\varepsilon\right)^{\frac{p-2}{2}}}\left[\delta \varphi(\varsigma) h^{2}\left(t^{p-1}\right)+K_{\delta}\right], \quad \text { in } \omega^{\prime}
$$

for every $t \geq 0$ and every $\varepsilon>0$ sufficiently small, for some constant $\kappa$ (possibly depending on $\zeta$ ) independent of $\delta$.

In the proof of Lemma 6.4 we make use of the Legendre transform of the function $h$. Here we recall its definition and some properties that we exploit in the following.

Given any convex function $h$, the Legendre transform is defined as

$$
h^{*}(y):=\sup _{r \in \mathbb{R}}[y r-h(r)] .
$$

Observe that, if $h \in C^{2}\left(\mathbb{R}^{+}\right)$, then $h^{*}$ is continuous, increasing and, due to the convexity of $h$, $h^{*}$ is convex, too. The generalized Young inequality reads as follows:

$$
\forall a, b \in \mathbb{R} \quad a b \leq h(|a|)+h^{*}(|b|) .
$$


We also remark that, being $h \in C^{2}\left(\mathbb{R}^{+}\right)$an increasing and convex function such that $h(0)=0$ and since (2.11) holds (i.e., roughly speaking, $h$ is a bit more than superlinear at infinity) it follows that $\lim _{s \rightarrow \infty} h^{\prime}(s)=+\infty$.

Consequently $h^{*-1}$ is well defined and $\lim _{y \rightarrow \infty} h^{*-1}(y)=+\infty$, too. Notice also that, since $h$ is smooth, $\forall y \geq 0$, we have

$$
h^{*}(y)=y\left[\left(h^{\prime}\right)^{-1}(y)\right]-h\left(\left(h^{\prime}\right)^{-1}(y)\right), \quad \text { so that } \quad h^{*}\left(h^{\prime}(y)\right)=y h^{\prime}(y)-h(y) .
$$

Proof. Let $\delta>0$ be fixed. First of all, we claim the existence of a function $\varphi=\varphi_{\delta}:[0,1] \rightarrow$ $[0,1]$ such that $\varphi$ belongs to $C^{0}([0,1]) \cap C^{2}(0,1)$ and it solves the Cauchy problem

$$
\left\{\begin{array}{l}
\varphi^{\prime}(s)=\sqrt{\delta} \varphi(s) h^{*-1}\left(\frac{C_{\delta}}{\sqrt{\delta \varphi(s)}}\right) \\
\varphi(0)=0
\end{array}\right.
$$

for some $C_{\delta}>0$, where $h^{*}$ denotes the Legendre transform of $h$ (see (6.28)). The existence of $\varphi$ can be deduced directly from [22, Lemma 2, by the change of unknown $\varphi=\psi^{2}$. Notice that the well-posedness of (6.30) relies on the integrability condition 2.11) since, by a direct computation, $\varphi$ is given by

$$
\int_{0}^{\sqrt{\delta \varphi(s)}} \frac{d t}{t\left(h^{*}\right)^{-1}\left(\frac{C_{\delta}}{t}\right)}=\frac{\sqrt{\delta}}{2} s, \quad \forall s>0 .
$$

The constant $C_{\delta}$ is set in order to have $\varphi(1)=1$. Observe that, since $\left(h^{*}\right)^{-1}\left(\frac{C_{\delta}}{t}\right) t$ is nondecreasing by the convexity of $h^{*}$, the condition $\varphi(1)=1$ implies

$$
\frac{1}{\left(h^{*}\right)^{-1}\left(\frac{C_{\delta}}{\sqrt{\delta}}\right)} \leq \frac{\sqrt{\delta}}{2}
$$

so $\left(h^{*}\right)^{-1}\left(\frac{C_{\delta}}{\sqrt{\delta}}\right) \rightarrow \infty$ as $\delta \rightarrow 0$.

Moreover $\lim _{s \rightarrow 0^{+}} \varphi^{\prime}(s)=0$. Indeed it is sufficient to notice directly from 6.30 that $\varphi(s)$ is twice differentiable (since $h(s) \in C^{2}(0,1)$ ) and that $\lim _{s \rightarrow 0^{+}} \varphi^{\prime}(s)=0$ (since $h^{*}$ is superlinear at infinity).

Now we claim that there exists a constant $\gamma$ (independent of $\delta$ ) such that

$$
\left|\varphi^{\prime \prime}(s)\right| \leq \gamma \frac{\varphi^{\prime 2}(s)}{\varphi(s)} .
$$

Observe that

$$
\varphi^{\prime \prime}(s)=\delta \varphi(s)\left(h^{*-1}\right)^{2}\left(\frac{C_{\delta}}{\sqrt{\delta \varphi(s)}}\right)-\left(h^{*-1}\right)^{\prime}\left(\frac{C_{\delta}}{\sqrt{\delta \varphi(s)}}\right) \frac{C_{\delta}}{2} \sqrt{\delta \varphi(s)} h^{*-1}\left(\frac{C_{\delta}}{\sqrt{\delta \varphi(s)}}\right)
$$

and consequently by 6.30 , we have that

$$
\frac{\varphi^{\prime \prime}(s)}{\varphi^{\prime}(s)}=\sqrt{\delta} h^{*-1}\left(\frac{C_{\delta}}{\sqrt{\delta \varphi(s)}}\right)-\frac{C_{\delta}}{2 \sqrt{\varphi(s)}}\left(h^{*-1}\right)^{\prime}\left(\frac{C_{\delta}}{\sqrt{\delta \varphi(s)}}\right),
$$

and thus

$$
\frac{\left|\varphi^{\prime \prime}(s)\right|}{\varphi^{\prime}(s)} \leq \frac{\varphi^{\prime}(s)}{\varphi(s)}+\left|\frac{C_{\delta}}{2 \sqrt{\varphi(s)}}\left(h^{*-1}\right)^{\prime}\left(\frac{C_{\delta}}{\sqrt{\delta \varphi(s)}}\right)\right|
$$


Hence we are done if we prove that

$$
\frac{C_{\delta}}{2 \sqrt{\varphi(s)}}\left(h^{*-1}\right)^{\prime}\left(\frac{C_{\delta}}{\sqrt{\delta \varphi(s)}}\right) \leq c \sqrt{\delta} h^{*-1}\left(\frac{C_{\delta}}{\sqrt{\delta \varphi(s)}}\right) \quad \forall s \in(0,1)
$$

or equivalently, performing the change of variable $h^{*}(\tau)=\frac{C_{\delta}}{\sqrt{\delta \varphi(s)}}$, if we prove that

$$
\frac{1}{2} \frac{h^{*}(\tau)}{\left(h^{*}\right)^{\prime}(\tau)} \leq c \tau \quad \forall \tau \geq h^{*-1}\left(C_{\delta} / \sqrt{\delta}\right)
$$

This is actually true because of the convexity of $h^{*}(\tau)$, so the claim is proved.

Finally, let us prove that 6.27 holds true. Let $\varsigma$ be any $W_{c}^{2, \infty}(\Omega)$ cut-off function such that $\operatorname{supp}(\varsigma)=\omega^{\prime}$ and let $\varphi$ be the function defined in 6.30 . Since

$$
\left|D^{2} \varphi\right| \leq\left|\varphi^{\prime \prime}(\varsigma)\right||D \varsigma|^{2}+\varphi^{\prime}(\varsigma)\left|D^{2} \varsigma\right| \leq c\left[\left|\varphi^{\prime \prime}(\varsigma)\right|+\varphi^{\prime}(\varsigma)\right],
$$

thanks to 6.32 we have

$$
t^{2}\left(t^{2}+\varepsilon\right)^{\frac{p-2}{2}}\left(\frac{|D \varphi|^{2}}{\varphi}+\left|D^{2} \varphi\right|\right) \leq c t^{2}\left(t^{2}+\varepsilon\right)^{\frac{p-2}{2}}\left[\gamma \frac{\varphi^{\prime 2}}{\varphi}+\varphi^{\prime}\right]
$$

Using (6.30) and 6.29), we have (for some constant $c$ independent of $\delta$ )

$$
\begin{gathered}
c \gamma t^{2}\left(t^{2}+\varepsilon\right)^{\frac{p-2}{2}} \frac{\varphi^{\prime 2}}{\varphi} \leq c \frac{\delta \varphi}{\left(t^{2}+\varepsilon\right)^{\frac{p-2}{2}}}\left[\left(t^{p-1}+1\right) h^{*-1}\left(\frac{C_{\delta}}{\sqrt{\delta \varphi}}\right)\right]^{2} \\
\leq c \frac{\delta \varphi}{\left(t^{2}+\varepsilon\right)^{\frac{p-2}{2}}}\left[h\left(t^{p-1}\right)+h(1)+2 \frac{C_{\delta}}{\sqrt{\delta \varphi(\varsigma)}}\right]^{2} \leq c\left(t^{2}+\varepsilon\right)^{\frac{p-2}{2}}\left\{\delta \varphi\left[h^{2}\left(t^{p-1}\right)+1\right]+C_{\delta}^{2}\right\} .
\end{gathered}
$$

Similarly, we have

$$
\begin{gathered}
t^{2}\left(t^{2}+\varepsilon\right)^{\frac{p-2}{2}} \varphi^{\prime} \leq \frac{\sqrt{\delta} \varphi}{\left(t^{2}+\varepsilon\right)^{\frac{p-2}{2}}}\left[\left(t^{2(p-1)}+1\right) h^{*-1}\left(\frac{C_{\delta}}{\sqrt{\delta \varphi}}\right)\right] \\
\leq \frac{\sqrt{\delta} \varphi}{\left(t^{2}+\varepsilon\right)^{\frac{p-2}{2}}}\left[t^{p-1} h\left(t^{p-1}\right)+h(1)+\left(t^{p-1}+1\right) \frac{C_{\delta}}{\sqrt{\delta \varphi(\varsigma)}}\right] \\
\leq c\left(t^{2}+\varepsilon\right)^{\frac{p-2}{2}}\left\{\sqrt{\delta} \varphi\left(t^{p-1} h\left(t^{p-1}\right)+1\right)+C_{\delta} \sqrt{\varphi}\left(t^{p-1}+1\right)\right\} .
\end{gathered}
$$

Therefore, overall we deduce

$$
\begin{gathered}
t^{2}\left(t^{2}+\varepsilon\right)^{\frac{p-2}{2}}\left(\frac{|D \varphi|^{2}}{\varphi}+\left|D^{2} \varphi\right|\right) \\
\leq \frac{c}{\left(t^{2}+\varepsilon\right)^{\frac{p-2}{2}}}\left\{\delta \varphi h^{2}\left(t^{p-1}\right)+\sqrt{\delta} \varphi+C_{\delta}^{2}+\sqrt{\delta} \varphi t^{p-1} h\left(t^{p-1}\right)+C_{\delta} \sqrt{\varphi}\left(t^{p-1}+1\right)\right\} .
\end{gathered}
$$

Since $h(s)$ is superlinear we have

$$
\sqrt{\delta} \varphi t^{p-1} h\left(t^{p-1}\right)+C_{\delta} \sqrt{\varphi} t^{p-1} \leq \delta \varphi h^{2}\left(t^{p-1}\right)+\tilde{C}_{\delta},
$$

for some $\tilde{C}_{\delta}>0$, and so the conclusion follows.

Thanks to the construction of the cut-off functions, we are now able to prove Theorem 2.5 .

\section{Proof of Theorem 2.5.}

First, let us fix $\omega \subset \subset \Omega$ and let us consider a cut-off function $\varphi \in W^{2, \infty}(\Omega)$ such that $\varphi \equiv 1$ on $\omega$ and $\operatorname{such}$ that $\operatorname{supp}(\varphi)=\omega^{\prime}, \omega \subset \subset \omega^{\prime} \subset \subset \Omega$.

Consequently, using the equation solved by $u_{\varepsilon}$ and since

$$
\left|D^{2} u_{\varepsilon}\right| \geq \eta \mathcal{A}_{\varepsilon, u_{\varepsilon}}\left(D^{2} u_{\varepsilon}\right), \quad \text { where } \quad \eta=\frac{1}{|p-2|+\sqrt{N}}
$$


we deduce that

$$
2 \varphi \alpha_{\varepsilon}\left(w_{\varepsilon}\right)\left|D^{2} u_{\varepsilon}\right|^{2} \geq 2 \eta^{2} \varphi \frac{\left[\lambda u_{\varepsilon}+H\left(x, D u_{\varepsilon}\right)\right]^{2}}{\alpha_{\varepsilon}\left(w_{\varepsilon}\right)} .
$$

Thus from (6.24) we deduce (we still denote by $c$ any constant independent from $\varepsilon$, whose value may vary from line to line),

$$
\begin{gathered}
2 \lambda z_{\varepsilon}-\alpha_{\varepsilon}\left(w_{\varepsilon}\right) \mathcal{A}_{\varepsilon, u_{\varepsilon}}\left(D^{2} z_{\varepsilon}\right)+D z_{\varepsilon} \cdot T_{\varepsilon}+2 \eta^{2} \varphi \frac{\left[\lambda u_{\varepsilon}+H\left(x, D u_{\varepsilon}\right)\right]^{2}}{\alpha_{\varepsilon}\left(w_{\varepsilon}\right)} \\
\leq 2 \varphi D u_{\varepsilon} \cdot H_{x}\left(x, D u_{\varepsilon}\right)+(p-2)\left|D u_{\varepsilon}\right||D \varphi|\left|\lambda u_{\varepsilon}+H\left(x, D u_{\varepsilon}\right)\right| \\
\left.+|D \varphi|\left|D u_{\varepsilon}\right|^{2}\left|H_{\xi}\left(x, D u_{\varepsilon}\right)\right|+c_{p}\left|D u_{\varepsilon}\right|^{2} \alpha_{\varepsilon}\left(w_{\varepsilon}\right)\left(\frac{|D \varphi|^{2}}{\varphi}+\mid D^{2} \varphi\right) \mid\right) \text { in } \omega^{\prime} .
\end{gathered}
$$

which implies, by Young inequality,

$$
\begin{gathered}
2 \lambda z_{\varepsilon}-\alpha_{\varepsilon}\left(w_{\varepsilon}\right) \mathcal{A}_{\varepsilon, u_{\varepsilon}}\left(D^{2} z_{\varepsilon}\right)+D z_{\varepsilon} \cdot T_{\varepsilon}+2(1-\delta) \eta^{2} \varphi \frac{\left[\lambda u_{\varepsilon}+H\left(x, D u_{\varepsilon}\right)\right]^{2}}{\alpha_{\varepsilon}\left(w_{\varepsilon}\right)} \\
\leq 2 \varphi D u_{\varepsilon} \cdot H_{x}\left(x, D u_{\varepsilon}\right)+|D \varphi|\left|D u_{\varepsilon}\right|^{2}\left|H_{\xi}\left(x, D u_{\varepsilon}\right)\right| \\
\left.+c\left|D u_{\varepsilon}\right|^{2} \alpha_{\varepsilon}\left(w_{\varepsilon}\right)\left(\frac{|D \varphi|^{2}}{\varphi}+\mid D^{2} \varphi\right) \mid\right) \quad \text { in } \omega^{\prime}
\end{gathered}
$$

where $\delta>0$ is a small parameter to be chosen later.

Similarly, from 2.12 and Young inequality, we have

$$
|D \varphi|\left|D u_{\varepsilon}\right|^{2}\left|H_{\xi}\left(x, D u_{\varepsilon}\right)\right| \leq 2 \delta \varphi \frac{\left|H\left(x, D u_{\varepsilon}\right)+1\right|^{2}}{\alpha_{\varepsilon}\left(w_{\varepsilon}\right)}+c_{\delta} \frac{|D \varphi|^{2}}{\varphi} w_{\varepsilon} \alpha_{\varepsilon}\left(w_{\varepsilon}\right) .
$$

Since, for $r \geq 1$, we have $(\varepsilon+r)^{\frac{p-2}{2}} \leq(1+\varepsilon)^{\frac{p-2}{2}} r^{\frac{p-2}{2}}$, using 2.13 we estimate

$$
2 \varphi D u_{\varepsilon} \cdot H_{x}\left(x, D u_{\varepsilon}\right) \leq 2 \varphi(1-\theta)(1+\varepsilon)^{\frac{p-2}{2}} \eta^{2} \frac{H^{2}\left(x, D u_{\varepsilon}\right)}{\alpha_{\varepsilon}\left(w_{\varepsilon}\right)}+c \varphi \text {. }
$$

Since

$$
\left|H\left(x, D u_{\varepsilon}\right)+\lambda u_{\varepsilon}\right|^{2} \geq(1-\delta)\left|H\left(x, D u_{\varepsilon}\right)\right|^{2}-c_{\delta}\left\|\lambda u_{\varepsilon}\right\|_{L^{\infty}\left(\omega^{\prime}\right)}^{2}
$$

we conclude that

$$
\begin{gathered}
2 \lambda z_{\varepsilon}-\alpha_{\varepsilon}\left(w_{\varepsilon}\right) \mathcal{A}_{\varepsilon, u_{\varepsilon}}\left(D^{2} z_{\varepsilon}\right)+D z_{\varepsilon} \cdot T_{\varepsilon} \\
+2 \varphi\left\{\left[(1-\delta)^{2}-(1-\theta)(1+\varepsilon)^{\frac{p-2}{2}}\right] \eta^{2}-\delta\right\} \frac{\left|H\left(x, D u_{\varepsilon}\right)\right|^{2}}{\alpha_{\varepsilon}\left(w_{\varepsilon}\right)} \\
\left.\leq c \eta^{2} \varphi \frac{\left\|\lambda u_{\varepsilon}\right\|_{L^{\infty}\left(\omega^{\prime}\right)}}{\alpha_{\varepsilon}\left(w_{\varepsilon}\right)}+c\left|D u_{\varepsilon}\right|^{2} \alpha_{\varepsilon}\left(w_{\varepsilon}\right)\left(\frac{|D \varphi|^{2}}{\varphi}+\mid D^{2} \varphi\right) \mid\right)+c \varphi .
\end{gathered}
$$

Choosing $\delta$ small enough (only depending on $\theta$ and $\eta$ ), for $\varepsilon$ sufficiently small the term in the second line is positive; therefore, on account of $(2.9)$, we deduce

$$
\begin{gathered}
2 \lambda z_{\varepsilon}-\alpha_{\varepsilon}\left(w_{\varepsilon}\right) \mathcal{A}_{\varepsilon, u_{\varepsilon}}\left(D^{2} z_{\varepsilon}\right)+D z_{\varepsilon} \cdot T_{\varepsilon}+\frac{\eta^{2}}{2} \varphi \frac{h^{2}\left(\left|D u_{\varepsilon}\right|^{p-1}\right)}{\alpha_{\varepsilon}\left(w_{\varepsilon}\right)} \\
\left.\leq c \varphi \frac{\left\|\lambda u_{\varepsilon}\right\|_{L^{\infty}\left(\omega^{\prime}\right)}+\|f\|_{L^{\infty}\left(\omega^{\prime}\right)}}{\alpha_{\varepsilon}\left(w_{\varepsilon}\right)}+c\left|D u_{\varepsilon}\right|^{2} \alpha_{\varepsilon}\left(w_{\varepsilon}\right)\left(\frac{|D \varphi|^{2}}{\varphi}+\mid D^{2} \varphi\right) \mid\right)+c \varphi,
\end{gathered}
$$

where the constants $c$ that appear, only depend on $p, N, \theta$.

We choose now $\varphi=\varphi(\zeta)$, where $\varphi$ is given by Lemma 6.4. For a suitable choice of $\delta$, sufficiently small, we deduce that $z_{\varepsilon}$ satisfies

$$
\begin{aligned}
2 \lambda z_{\varepsilon} & -\alpha_{\varepsilon}\left(w_{\varepsilon}\right) \mathcal{A}_{\varepsilon, u_{\varepsilon}}\left(D^{2} z_{\varepsilon}\right)+D z_{\varepsilon} \cdot T_{\varepsilon}+\frac{\eta^{2}}{4} \varphi \frac{h^{2}\left(\left|D u_{\varepsilon}\right|^{p-1}\right)}{\alpha_{\varepsilon}\left(w_{\varepsilon}\right)} \\
& \leq c \varphi \frac{\left\|\lambda u_{\varepsilon}\right\|_{L^{\infty}\left(\omega^{\prime}\right)}+\|f\|_{L^{\infty}\left(\omega^{\prime}\right)}}{\alpha_{\varepsilon}\left(w_{\varepsilon}\right)}+\frac{c}{\alpha_{\varepsilon}\left(w_{\varepsilon}\right)}+c \varphi .
\end{aligned}
$$


Now we use the maximum principle, and since $z_{\varepsilon}=0$ at $\partial \omega^{\prime}$ we deduce that the inequality

$$
\varphi \frac{h^{2}\left(\left|D u_{\varepsilon}\right|^{p-1}\right)}{\alpha_{\varepsilon}\left(w_{\varepsilon}\right)} \leq c \varphi \frac{\left\|\lambda u_{\varepsilon}\right\|_{L^{\infty}\left(\omega^{\prime}\right)}+\|f\|_{L^{\infty}\left(\omega^{\prime}\right)}}{\alpha_{\varepsilon}\left(w_{\varepsilon}\right)}+\frac{c}{\alpha_{\varepsilon}\left(w_{\varepsilon}\right)}+c \varphi
$$

must hold at the maximum point of $z_{\varepsilon}$. This means that, for some constant $K$ (depending on $\left.\left\|\lambda u_{\varepsilon}\right\|_{L^{\infty}\left(\omega^{\prime}\right)},\|f\|_{L^{\infty}\left(\omega^{\prime}\right)}\right)$ it holds

$$
h^{2}\left(\left|D u_{\varepsilon}\right|^{p-1}\right) \leq K+\frac{c}{\varphi}
$$

and in particular, being $h$ superlinear and $p \geq 2$,

$$
\max _{\omega^{\prime}} \varphi\left|D u_{\varepsilon}\right|^{2} \leq \varphi\left(\left|D u_{\varepsilon}\right|^{2(p-1)}+1\right) \leq \tilde{K}
$$

that is $z_{\varepsilon}$ is uniformly bounded. Since $\varphi(\zeta) \equiv 1$ on $\omega$, estimate 2.15 holds.

\section{NEUMANN BOUNDARY CONDITIONS}

Similar gradient bounds as developed before can be applied to the solvability of the Neumann problem associated to $\left(E_{\lambda}\right.$. For simplicity, we only discuss the case $\lambda>0$.

As observed in [29], the Neumann condition may be used to easily estimate the maximum of the gradient at the boundary. This implies that we have a complete control of the gradient in the whole $\bar{\Omega}$, just using the interior bound.

As before, we consider the approximating elliptic equation $(6.9)$ complemented now with the Neumann condition $\frac{\partial u_{\varepsilon}}{\partial \nu}=0$ at $\partial \Omega$. The structure conditions $\left.(2.4)-2.6\right)$ are also conveniently modified adapted to the Neumann problem. More precisely, we suppose that there exist $\theta \in(0,1)$ and $\rho, c, \delta_{0}>0$ such that for any $\delta \in\left(0, \delta_{0}\right)$ we have

$$
\begin{aligned}
\liminf _{|\xi| \rightarrow+\infty} \xi \cdot H_{x}(x, \xi)+ & (1-\theta) \eta^{2}|\xi|^{2-p} H^{2}(x, \xi)+t|\xi|^{2} H_{\xi}(x, \xi) \cdot D d \\
& +\delta\left\{\rho|\xi|^{p+2}+\tau|\xi|^{2}\left[\xi \cdot H_{\xi}(x, \xi)-(p-1) H(x, \xi)\right]\right\} \geq 0,
\end{aligned}
$$

uniformly with respect to $x \in \Omega$ and $t \in\left[0, t_{0}\right]$ with $t_{0}$ depending on the geometry of $\Omega$, where $\eta=\frac{1}{|p-2|+\sqrt{N}}$ and $\tau$ is either 1 or -1 . Analogously, we naturally modify 2.6 adapting it for the Neumann boundary conditions:

there exist $\theta \in(0,1)$ and $\rho, c, \delta_{0}>0$ such that for any $\delta \in\left(0, \delta_{0}\right)$ we have

$$
\begin{aligned}
& \liminf _{|\xi| \rightarrow+\infty} \xi \cdot H_{x}(x, \xi)+(1-\theta) \eta^{2}|\xi|^{2-p} H^{2}(x, \xi)+t|\xi|^{2} H_{\xi}(x, \xi) \cdot D d \\
&+\delta\left\{\rho|\xi|^{p+2}-|\xi|^{2}\left|\xi \cdot H_{\xi}(x, \xi)-(p-1) H(x, \xi)\right|\right\} \geq 0,
\end{aligned}
$$

uniformly with respect to $x \in \Omega$ and $t \in\left[0, t_{0}\right]$, with $t_{0}$ depending on the geometry of $\Omega$, and where $\eta=\frac{1}{|p-2|+\sqrt{N}}$.

Here we state our a priori estimate result.

Proposition 7.1. Assume that $H(x, \xi)$ satisfies (2.2) and that (2.4) and (7.34) or (7.35) are satisfied. Then for any $\lambda>0$ there exists a constant $c$ independent of $\varepsilon$ such that any bounded solution of $(6.9)$ that satisfies $\frac{\partial u_{\varepsilon}}{\partial \nu}=0$ at $\partial \Omega$, is such that $\sup _{\bar{\Omega}}\left|D u_{\varepsilon}\right| \leq c$.

Proof. We only sketch the proof, which is a variation of Theorem 2.1. Following [29] (see also [24]), we define

$$
z_{\varepsilon}=\left(1+\beta\left(u_{\varepsilon}\right)\right)\left|D u_{\varepsilon}\right|^{2} e^{\phi(d)}
$$


where $\phi$ is a $C^{2}[0, \operatorname{diam} \Omega)$ function which is properly chosen so that the maximum of $z_{\varepsilon}$ is not achieved at $\partial \Omega$ due to the Neumann condition over $u_{\varepsilon}$. Indeed, the proof is based on two main facts:

(1) Using $\frac{\partial u_{\varepsilon}}{\partial \nu}=0$ at $\partial \Omega$, we deduce (see e.g. [23, Lemma 2.4]) that, if $\phi$ is increasing and $\phi^{\prime}(0) \geq 2\left\|D^{2} d\right\|_{L^{\infty}(\partial \Omega)}$, then

$$
\frac{\partial z_{\varepsilon}}{\partial \nu}<0 \quad \text { at } \partial \Omega
$$

Thus any maximum point of $z_{\varepsilon}$ is not attained at the boundary (Hopf Lemma).

(2) According to 6.21) we deduce (with the notations of Proposition 6.2 and Theorem 2.1 that $z_{\varepsilon}$ is a subsolution to

$$
\begin{aligned}
& -\tilde{\mathcal{L}}_{\varepsilon}\left(z_{\varepsilon}\right)+2 \lambda z_{\varepsilon}=-2(1-\theta) \eta^{2}\left(1+\beta\left(u_{\varepsilon}\right)\right) \frac{H^{2}\left(x, D u_{\varepsilon}\right)}{\alpha_{\varepsilon}\left(w_{\varepsilon}\right)} e^{\phi(d)} \\
& -\mu\left|D u_{\varepsilon}\right|^{2}\left[\rho\left|D u_{\varepsilon}\right|^{p}+\left(D u_{\varepsilon} \cdot H_{\xi}\left(x, D u_{\varepsilon}\right)-(p-1) H\left(x, D u_{\varepsilon}\right)\right)\right] e^{\phi(d)} \\
& -2\left(1+\beta\left(u_{\varepsilon}\right)\right) D u_{\varepsilon} \cdot H_{x}\left(x, D u_{\varepsilon}\right) e^{\phi(d)} \\
& +\left|\beta^{\prime}\left(u_{\varepsilon}\right)\right| w_{\varepsilon}\left[1+\frac{(p-2) w_{\varepsilon}}{\varepsilon+w_{\varepsilon}}\right]\left\|\lambda u_{\varepsilon}\right\|_{L^{\infty}(\Omega)} e^{\phi(d)}+c_{\theta} \eta^{2}\left(1+\beta\left(u_{\varepsilon}\right)\right) \frac{\left\|\lambda u_{\varepsilon}\right\|_{L^{\infty}(\Omega)}^{2}}{\alpha_{\varepsilon}\left(w_{\varepsilon}\right)} e^{\phi(d)} \\
& +\alpha_{\varepsilon}\left(w_{\varepsilon}\right) z_{\varepsilon}\left[\phi^{\prime}(d) \mathcal{A}_{\varepsilon, u_{\varepsilon}}\left(D^{2} d\right)+\mathcal{A}_{\varepsilon, u_{\varepsilon}}(D d \otimes D d)\left(\phi^{\prime 2}(d)-\phi^{\prime \prime}(d)\right)\right]-\phi^{\prime}(d) z_{\varepsilon} D d \cdot \tilde{T}_{\varepsilon}, \\
& -\tilde{\mathcal{L}}_{\varepsilon}\left(z_{\varepsilon}\right)=-\alpha_{\varepsilon}\left(w_{\varepsilon}\right) \mathcal{A}_{\varepsilon, u_{\varepsilon}}\left(D^{2} z_{\varepsilon}\right)+\tilde{T}_{\varepsilon} \cdot D z_{\varepsilon} \\
& \tilde{T}(x)=2 \alpha_{\varepsilon}\left(w_{\varepsilon}\right) \frac{\beta^{\prime}\left(u_{\varepsilon}\right)}{1+\beta\left(u_{\varepsilon}\right)}\left[1+(p-2) \frac{w_{\varepsilon}}{\varepsilon+w_{\varepsilon}}\right] D u_{\varepsilon}+H_{\xi}\left(x, D u_{\varepsilon}\right)-2 \alpha_{\varepsilon}^{\prime}\left(w_{\varepsilon}\right) \mathcal{A}_{\varepsilon, u_{\varepsilon}}\left(D^{2} u_{\varepsilon}\right) D u_{\varepsilon} \\
& +\frac{(p-2)}{\left(1+\beta\left(u_{\varepsilon}\right)\right)^{2}} \frac{\alpha_{\varepsilon}\left(w_{\varepsilon}\right)}{\left(\varepsilon+w_{\varepsilon}\right)^{2}}\left(\left[D v_{\varepsilon} \cdot D u_{\varepsilon}\right] D u_{\varepsilon}-2 w_{\varepsilon}^{2} \beta^{\prime}\left(u_{\varepsilon}\right) D u_{\varepsilon}-\frac{1}{2}\left(\varepsilon+w_{\varepsilon}\right) D v_{\varepsilon}+w_{\varepsilon} \beta^{\prime}\left(u_{\varepsilon}\right) D u_{\varepsilon}\right) \\
& -(p-2) \frac{D u_{\varepsilon} \cdot D d}{\varepsilon+\left|D u_{\varepsilon}\right|^{2}} D u_{\varepsilon} \text {. }
\end{aligned}
$$

Eventually, arguing as in the proof of Theorem 2.1, we deduce that if $x_{0}$ is any interior maximum point, then $z_{\varepsilon}\left(x_{0}\right)$ is uniformly bounded, if either $(2.3)$ and $(7.34)$ or $(7.35)$ hold true.

A direct consequence of the global gradient bound is the following existence result for Lipschitz solutions of

$$
\begin{cases}\lambda u-\Delta_{p} u+H(x, D u)=0 & \text { in } \Omega, \\ \frac{\partial u}{\partial \nu}=0 . & \text { on } \partial \Omega .\end{cases}
$$

Theorem 7.2. Assume that $H(x, \xi)$ satisfies (2.2) and that (2.4)-(7.34) or (7.35) are satisfied. Then for any $\lambda>0$ there exists a unique solution $u \in W^{1, \infty}(\Omega)$ of problem 7.36).

Proof. Uniqueness. We argue as in Theorem 2.3 [25] (see Proposition 2.1 therein). Assume that $u, v \in W^{1, \infty}(\Omega)$ are solutions of (7.36), and define, for any $\delta>0$,

$$
\begin{cases}\psi_{\delta}(s)=e^{-\frac{1}{\delta} \frac{1}{p-2} \frac{1}{s^{p-2}}} & \text { if } s>0 \\ \psi_{\delta}(0)=0 . & \end{cases}
$$

Then if we choose $\varphi_{\delta}(u-v)^{+}$, where for as test functions in the formulations of $u$ and $v$, respectively, and we subtract each other, we get (we denote $z=u-v$ ), using the homogeneous Neumann boundary conditions,

$$
\lambda \int_{\Omega} z \psi_{\delta}\left(z^{+}\right)+\frac{1}{\delta} \int_{\Omega} \psi_{\delta}\left(z^{+}\right) \frac{|D z|^{p}}{z^{p-1}} \leq \beta \int_{\Omega} \psi_{\delta}\left(z^{+}\right)|D z|, \quad \beta>0 .
$$


We easily deduce, choosing $\delta$ small enough that $u \leq v$ in $\Omega$. Interchanging the role of $u$ and $v$ we get uniqueness.

Existence. Let $u_{\varepsilon}$ be a solution of $(6.9)$, with $\lambda>0$, that satisfies the boundary condition

$$
\frac{\partial u_{\varepsilon}}{\partial \nu}=0 \quad \text { at } \partial \Omega
$$

(its existence and regularity is guaranteed by Theorem 2 in [26]). too.

Moreover $\left\|u_{\varepsilon}\right\|_{L^{\infty}(\Omega)} \leq \frac{\|H(x, 0)\|_{L^{\infty}(\Omega)}}{\lambda}$ and, thanks to Theorem 7.1. $\left\|D u_{\varepsilon}\right\|_{L^{\infty}(\Omega)}$ is bounded,

This is enough to pass to the limit and find a solution of (7.36).

Acknowledgements. The authors have been supported by Projects MTM2012-31799 (MINECO, Spain), FQM-116, (Junta de Andalucía, Spain) and by Fondation Sciences Mathematique de Paris.

\section{REFERENCES}

[1] B. Abdellaoui, A. Dall'Aglio, I. Peral; Some remarks on elliptic problems with critical growth in the gradient J. Differential Equations 222 (2006) 21-62.

[2] S. Alarcón, J. García-Melián, A. Quaas; Existence and non-existence of solutions to elliptic equations with a general convection term, Proc. Roy. Soc. Edinburgh Sect. A 144 (2014), 225-239.

[3] A. Attouchi; Well-posedness and gradient blow-up estimate near the boundary for a Hamilton-Jacobi equation with degenerate diffusion. Journal of Differential Equations 253 (2012) 2474-2492.

[4] A. Attouchi; Gradient estimate and apllication to a Liouville theorem for a p-Laplacian evolution equation with a gradient nonlinearity, Preprint (Available at http://arxiv.org/pdf/1405.5896v1.pdf)

[5] A. Attouchi, G. Barles; Global Continuation beyond Singularities on the Boundary for a Degenerate Diffusive Hamilton-Jacobi Equation, Preprint (Available at http://arxiv.org/pdf/1311.3593.pdf).

[6] G. Barles; A weak Bernstein method for fully nonlinear elliptic equations, Differential Integral Equations 4 (1991), 241-262.

[7] G.Barles; A short proof of the $C^{0, \alpha}$-regularity of viscosity subsolutions for superquadratic viscous HamiltonJacobi equations and applications, Nonlinear Anal. 73 (2010), 31-47.

[8] G.Barles, F.Murat; Uniqueness and the maximum principle for quasilinear elliptic equations with quadratic growth conditions, Arch. Rational Mech. Anal. 133 (1995), 77-101.

[9] G.Barles, A.Porretta; Uniqueness for unbounded solutions to stationary viscous Hamilton-Jacobi equations, Ann. Sc. Norm. Super. Pisa Cl. Sci. 5 (2006), 107-136.

[10] G.Barles, A.Porretta, T.T. Tchamba; On the large time behavior of solutions of the Dirichlet problem for subquadratic viscous Hamilton-Jacobi equations J. Math. Pures Appl. 94 (2010), 497-519.

[11] J-P. Bartier, P. Laurençot; Gradient estimates fora degenerate parabolic equation with gradient absorption and applications, J. Funct. Anal. 254 (2008), 851-878.

[12] S. Bernstein; Sur la généralization du probléme de Dirichlet II, Math. Ann. 69 (1910), 82-136.

[13] L. Boccardo, F. Murat, J.P. Puel; $L^{\infty}$ estimate for some nonlinear elliptic partial differential equations and application to an existence result, SIAM J. Math. Anal. 23 (1992), n. 2, 326-333.

[14] I. Capuzzo Dolcetta, F. Leoni, A. Porretta; Hölder estimates for degenerate elliptic equations with coercive Hamiltonians, Trans. Amer. Math. Soc. 362 (2010), 4511-4536.

[15] A.Dall'Aglio, A.Porretta; Local and global regularity of weak solutions of elliptic equations with superquadratic Hamiltonian, Trans. Amer. Math. Soc. 367 (2015), 3017-3039.

[16] E. DiBenedetto; $C^{1+\alpha}$ local regularity of weak solutions of degenerate elliptic equations, Nonlinear Anal. 7 (1983), 827-850.

[17] V. Ferone, E. Giarrusso, B. Messano, M. R. Posteraro, Isoperimetric inequalities for an ergodic stochastic control problem Calc. Var. 46 (2013) 749-768

[18] D. Gilbarg, N. Trudinger; Partial Differential equations of Second Order, 2nd ed., Springer-Verlag, Berlin/New-York (1983).

[19] T. Kilpeläinen, H. Shahgholian, X. Zhong; Growth estimates through scaling for quasilinear partial differential equations Annales Academiae Scientiarum Fennicae Mathematica 32 (2007) 595-599

[20] J.M. Lasry, P.-L. Lions; Nonlinear elliptic equations with singular boundary conditions and stochastic control with state constraints. I. The model problem, Math. Ann. 283 (1989), 583-630. 
[21] T. Leonori; Large solutions for a class of nonlinear elliptic equations with gradient terms, Adv. Nonlin. Stud. 7 (2007), 237-269.

[22] T. Leonori, F.Petitta; Local estimates for parabolic equations with nonlinear gradient terms, Calc.Var. 42 (2011) 153-187.

[23] T. Leonori, A. Porretta; The boundary behavior of blow-up solutions related to a state constraint problem, SIAM Math. Anal. 39 (2008), 1295-1327.

[24] T. Leonori, A. Porretta; Gradient bounds for elliptic problems singular at the boundary, Arch. Rational Mech. Anal. 202 (2011), 663-705.

[25] T. Leonori, A. Porretta, G.Riey; Comparison principles for p-Laplace equations with lower order terms, Preprint.

[26] G. Lieberman; Boundary regularity for solutions of degenerate elliptic equations, Nonlinear Anal. 12 (1988), $1203-1219$.

[27] G. Lieberman; Elliptic equations with strongly singular lower order terms, Indiana Univ. Math. J. 57 (2008), 2097-2135.

[28] P.-L. Lions; Generalized solutions of Hamilton-Jacobi equations, Research Notes in Mathematics, 69. Pitman, Boston 1982.

[29] P.-L. Lions; Résolution de problèmes elliptiques quasilinéaires, Arch. Rat. Mech. An. 74 (1980), $234-254$.

[30] P.-L. Lions; Quelques remarques sur les problémes elliptiques quasilinèaires du second ordre, J. Anal. Math. 45 (1985), 335-353.

[31] A. Porretta; On the comparison principle for p-Laplace type operators with rst order terms, in "On the notions of solution to nonlinear elliptic problems: results and developments", 459-497, Quad. Mat. 23, Dept. Math., Seconda Univ. Napoli, Caserta (2008)

[32] A. Porretta; The "ergodic limit" for a viscous Hamilton-Jacobi equation with Dirichlet conditions, Atti Accad. Naz. Lincei Cl. Sci. Fis. Mat. Natur. Rend. Lincei (9) Mat. Appl. 21 (2010), 59-78.

[33] A. Porretta, L.Veron; Separable p-harmonic functions in a cone and related quasilinear equations on manifolds J. Eur. Math. Soc. (JEMS) 11 (2009), 1285-1305.

[34] J. Serrin; The problem of Dirichlet for quasilinear elliptic differential equations with many independent variables, Philos. Trans. Roy. Soc. London Ser. A 264 (1969), 413-496.

[35] J. Serrin; Gradient estimates for solutions of nonlinear elliptic and parabolic equations, Contributions to nonlinear functional analysis (Edit. by E.H. Zarandello) 565-601. New York: Academic Press 1971.

[36] G. Stampacchia, Le problème de Dirichlet pour les équations elliptiques du seconde ordre à coefficientes discontinus, Ann. Inst. Fourier (Grenoble), 15 (1965), 189-258.

[37] T. T. Tchamba, Large Time behavior of Solutions of Viscous Hamilton-Jacobi Equations with Superquadratic Hamiltonian, Asymptot. Analysis 66 (2010) 161-186.

TOMMASO LEONORI

Departamento de Análisis Matemático Universidad de Granada, Campus Fuentenueva S/N, 18071, Granada, España

E-mail address: leonori@ugr.es

Alessio Porretta

Dipartimento di Matematica, Università di Roma "Tor Vergata", Via della Ricerca Scientifica 1, 00133 Roma, Italia

E-mail address: porretta@mat.uniroma2.it 